Portland State University

PDXScholar

Spring 6-6-2019

\title{
Physics-Based Signal Processing Methods for Terahertz Non-Destructive Evaluation of Layered Media
}

Scott G. Schecklman

Portland State University

Follow this and additional works at: https://pdxscholar.library.pdx.edu/open_access_etds

Part of the Electrical and Computer Engineering Commons Let us know how access to this document benefits you.

\section{Recommended Citation}

Schecklman, Scott G., "Physics-Based Signal Processing Methods for Terahertz Non-Destructive Evaluation of Layered Media" (2019). Dissertations and Theses. Paper 5013.

https://doi.org/10.15760/etd.6889

This Dissertation is brought to you for free and open access. It has been accepted for inclusion in Dissertations and Theses by an authorized administrator of PDXScholar. Please contact us if we can make this document more accessible: pdxscholar@pdx.edu. 


\title{
Physics-Based Signal Processing Methods
}

for Terahertz Non-Destructive Evaluation of Layered Media

by

Scott G. Schecklman

A dissertation submitted in partial fulfillment of the requirements for the degree of

\author{
Doctor of Philosophy \\ in \\ Electrical and Computer Engineering
}

\author{
Dissertation Committee: \\ Lisa M. Zurk, Chair \\ T. Martin Siderius \\ Branimir Pejcinovic \\ Suresh Singh
}

Portland State University

2019 
(c) 2019 Scott G. Schecklman 


\begin{abstract}
In recent years Terahertz $(\mathrm{THz})$ time domain spectroscopy has emerged as a promising new technology with potential applications in a variety of fields, including industrial manufacturing, security screening and medical imaging. Pulsed $\mathrm{THz}$ systems are uniquely suited for non-destructive evaluation (NDE) of the sub-surface layers of dielectric packaging and coating materials, because they provide high dynamic range over a wide bandwidth in the far infrared portion of the electromagnetic spectrum. Often the dielectric materials of the packaging and/or surface coating layers exhibit relatively low loss and abrupt changes in the refractive index at the layer boundaries can be observed as a train of $\mathrm{THz}$ pulses in A-scan data.
\end{abstract}

However, many practical applications of $\mathrm{THz}$ NDE will require fast signal acquisition to efficiently scan and evaluate many samples. The conventional processing approach shown in much of the published work in the field of THz NDE does not perform well in low signal-to-noise ratio (SNR) conditions. In addition, many samples of interest contain thin film layers and the $\mathrm{THz}$ pulses reflecting from the boundaries overlap on top of one another. Thus, it is not always possible to calculate the thickness of thin films from conventional time difference of arrival (TDOA) measurements.

In this dissertation physics-based signal processing methods that have been historically used for radar/sonar signal processing are adapted and applied for THz NDE of layered media. Results are demonstrated with measured data from a pulsed THz system in the Northwest Electromagnetic and Acoustics Research Laboratory (NEARLab) at Portland State University (PSU). 
This research is expected to provide an important link for $\mathrm{THz}$ researchers to access and apply the robust methods that have been developed over several decades for other applications.

Two key contributions of this work are:

1. Development of a matched filter approach for THz NDE of thick layered media based on the maximum likelihood estimator (MLE).

2. Development of a matched field processing (MFP) approach for THz NDE of thin-film layered media, based on techniques in the underwater acoustics literature. 


\section{Acknowledgments}

This work would not have been possible without the support of many people.

First, I would like to thank my adviser, Dr. Lisa Zurk for her many years of guidance in the study of Terahertz and physics-based signal processing during my masters degree and in my early research career. I'm grateful for the opportunity to be her co-author for a book chapter about THz scattering from random media, and to be her co-PI during a 5-year grant from the National Science Foundation (NSF). Dr. Zurk challenged me to continually improve and grow in the pursuit of excellence in all aspects of my work. Along the way she also provided many opportunities to present my work at professional research conferences, to publish several articles in technical journals and ultimately to pursue this $\mathrm{PhD}$.

Thanks are also due to all of the members of my dissertation committee for their time and support during the development of this research. I'd like to thank Dr. Martin Siderius for his support in my education and career, and especially for helpful conversations about matched field processing techniques in the field of underwater ocean acoustics. I'm also thankful to Dr. Branimir Pejcinovic and Dr. Suresh Singh for their many helpful discussions about the design of $\mathrm{THz}$ laboratory experiments and interpretation of results. 
Many thanks are also due to Dr. Richard Campbell for his many inspirational and educational lectures in various electromagnetics courses during my all phases of my education, including my bachelors degree at Michigan Technical University as well as my masters and PhD degrees at Portland State University.

I would like to thank all the members of the Northwest Electromagnetics and Acoustics Research Laboratory (NEAR-Lab) for countless hours of technical discussions about physics-based signal processing concepts going back to 2007. In particular, I enjoyed working with Dr. Gabe Kniffin and Dr. Sam Henry as we explored new experimental methods in the THz laboratory. Thanks also to John Gebbie for many insightful discussions about model-based parameter estimation techniques for underwater acoustic applications.

Thanks are also due to my employer, Metron Inc., for providing much of the financial support for this PhD work. In particular I am grateful to Dr. Bob Zarnich, manager of the Advanced Mathematics Applications (AMA) division, for allowing me to have have a flexible work schedule while completing my dissertation. I'd also like to thank NSF for funding much of the NEAR-Lab's THz hardware and experimental sample materials used in this research as well as some of the underlying research studies.

Finally, a special thank you to my wife, Marianne, for her eternal optimism. She patiently provided the support and encouragement needed to complete this work. 


\section{Table of Contents}

Abstract $\quad$ i

Acknowledgments $\quad$ iii

List of Tables $\quad$ X

List of Figures $\quad$ xi

Chapter 1 Introduction and Motivation 1

1.1 Introduction . . . . . . . . . . . . . . . . . . 1

1.2 Pulsed $\mathrm{THz}$ Systems . . . . . . . . . . . . . . . . . . . . 2

1.3 Potential Applications for Terahertz Technology . . . . . . . . . . . . 6

1.4 Challenges in Terahertz Non-Destructive Evaluation . . . . . . . . . . 7

1.4.1 Low SNR in Fast THz Scans of Thick Layers . . . . . . . . . . 9 
1.4.2 Overlapping THz Pulses in Thin Films . . . . . . . . . . . . . 9

1.5 Physics-Based Signal Processing Approach . . . . . . . . . . . . . 10

1.5.1 Derivation of the Matched Filter for Low-SNR Applications . 10

1.5.2 Adaptation of Matched Field Processing Techniques for Thin Films ........................... 12

1.6 Contributions and Significance of this Work . . . . . . . . . . 14

$\begin{array}{lll}\text { Chapter } 2 & \text { Literature Review } & 16\end{array}$

$2.1 \mathrm{THz}$ Non-Destructive Evaluation of Layered Media . . . . . . . . . 16

2.2 Matched Field Processing . . . . . . . . . . . . . . 19

$\begin{array}{lll}\text { Chapter } 3 & \text { Theoretical Background } & 26\end{array}$

$3.1 \mathrm{THz}$ Time Domain Spectroscopy . . . . . . . . . . . . . 26

3.1.1 THz Spectroscopy for a Thick Layer . . . . . . . . . . . . . . 30

3.1.2 THz Spectroscopy for a Film Layer on a Substrate . . . . . . 33

$3.2 \mathrm{THz}$ NDE of Thick Layered Media . . . . . . . . . . . . . 35

3.2.1 Conventional Method: Deconvolution Processing . . . . . . . . 37

3.2.2 Proposed Approach: Matched Filter . . . . . . . . . . . . . 39 
$3.3 \mathrm{THz}$ NDE of Thin Films $\ldots \ldots \ldots \ldots \ldots$

3.3.1 Matched Field Processing (MFP) Approach . . . . . . . . . 44

3.3.2 Measurement Data from Pulsed $\mathrm{THz}$ Sensors . . . . . . . . . . 44

3.3.3 Mathematical Model for Terahertz Measurement Data . . . 45

3.3.4 Generating THz Replica Spectra . . . . . . . . . . . . 48

3.3.5 Sample Covariance Matrix . . . . . . . . . . . 50

3.3 .6 Objective Functions $\ldots \ldots \ldots \ldots \ldots$

3.3.6.1 Bartlett Processor . . . . . . . . . . . . . 51

3.3.6.2 Minimum Variance (MV) Processor . . . . . . . . . 52

3.3 .7 Ambiguity Surfaces . . . . . . . . . . . . . . . 53

Chapter 4 Implementation, Demonstration, and Analysis $\quad 54$

4.1 THz NDE of Thick Layered Media . . . . . . . . . . . 55

4.1.1 Experiment Samples and Measurement Configuration . . . . 57

4.1.2 THz Signal Processing Results: A-Scans _ . . . . . . . . . . 59

4.1.3 THz Image Processing Results: B-Scans . . . . . . . . . . . 61

4.1.4 Receiver Operating Characteristic (ROC) Curves . . . . . 65 
$4.2 \mathrm{THz}$ NDE of Thin Films of Air in Polycarbonate . . . . . . . . . . 68

4.2.1 Terahertz Measurement System . . . . . . . . . . . . . . . 70

4.2.2 Layered Media Samples . . . . . . . . . . . . . . . . . . . . 72

4.2.3 Measurement Data Processing . . . . . . . . . . . . . . 75

4.2.4 Generating Replica Spectra . . . . . . . . . . . . . 78

4.2.5 THz MFP Results for Sample A . . . . . . . . . . . . . . 81

4.2.6 THz MFP Results for Sample D . . . . . . . . . . . . . . . . 84

4.2.7 Error Analysis . . . . . . . . . . . . . . . . 86

$4.3 \mathrm{THz}$ NDE for Thin Films of Acrylic Paint . . . . . . . . . . . . . 88

4.3.1 Terahertz Measurement System . . . . . . . . . . . . . . 90

4.3.2 Paint Film Samples . . . . . . . . . . . . . . . . . . . . . . . 91

4.3.3 Measurement Data Processing . . . . . . . . . . . . . . . 97

4.3.4 Generating Replica Spectra . . . . . . . . . . . . . . 99

4.3.5 THz MFP Results for Paint Film Samples . . . . . . . . . . . 102

4.3 .6 Error Analysis . . . . . . . . . . . . . . . . . . . . . . 104

$\begin{array}{lll}\text { Chapter } 5 & \text { Conclusions and Future Work } & 114\end{array}$ 
5.1 Broader Impacts and Future Work _. . . . . . . . . . . . 115

5.2 Publications and Conference Presentations . . . . . . . . . . . 118

Bibliography

Appendix A Propagation Model for Plane Waves in Layered Media 132

A.1 Introduction . . . . . . . . . . . . . . . . . . . 132

A.2 Transfer Matrix Model . . . . . . . . . . . . . . . . . . . 133

Appendix B MFP Results for Air Film in Polycarbonate

B.1 Sample Covariance Matrices . . . . . . . . . . . . . . . 137

B.2 Ambiguity Surfaces . . . . . . . . . . . . . . . . . . . 142 


\section{List of Tables}

1.3.1 THz NDE offers advantages over conventional methods. . . . . . . . 6

4.2.1 Four samples of thin polycarbonate films (layer $d_{1}$ in Figure 4.2.1) were evaluated in this study. . . . . . . . . . . . . . . . . . .

4.2.2 Thickness estimates obtained from THz MFP with the Bartlett and MV objective functions for the experiment configuration shown in Figure 4.2.1. All of the differences between the $\mathrm{THz} \mathrm{MFP}$ results and the Vernier caliper measurements are within the measurement resolution of

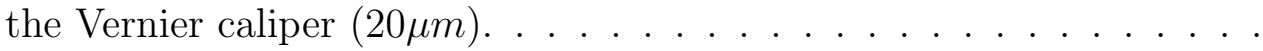

4.3.1 Six (6) samples (A - F) of Dupli-Color@Perfect Match®paint film on aluminum substrate were measured with the CEM DT-156 coating thickness tester [62] shown in Figure 4.3.4. Thirty (30) measurements for each sample were used to compute the statistics shown. This data is used to evaluate the accuracy of the $\mathrm{THz}$ MFP results presented later in this section. . . . . . . . . . . . . . . . . . . Thickness estimates obtained from $\mathrm{THz}$ MFP with the Bartlett and MV objective functions for the experiment configuration shown in Figure 4.3.1. All of the differences between the $\mathrm{THz} \mathrm{MFP}$ results and the CEM DT-156 Coating Thickness Tester measurements are within the uncertainty values for the Coating Thickness Tester. . . . . . . . . 


\section{List of Figures}

1.1.1 The terahertz gap is a portion of the electromagnetic spectrum between microwave (radio) and optical frequencies. Electronics and photonics technologies have developed on either side of this gap, but were not able to reach into this region of the spectrum until recently. . . . . . . . .

1.2.1 The T-Ray 4000 is used for THz NDE research in the Northwest Electromagnetics and Acoustics Research (NEAR) Lab at Portland State University (PSU). It was developed and manufactured by Picometrix Inc. 4

1.2.2 (a) A THz Pulse measured with the Picometrix T-Ray 4000 at the NEAR-Lab. The pulse duration is approximately 5 picoseconds (ps). (b) The power spectral density (PSD) of the pulse shown in figure 1.2.2a. The usable portion of the spectrum spans approximately from 0.1 to 2 THz. . . . . . . . . . . . . . . . . . . .

1.4.1 Two primary challenges for $\mathrm{THz}$ NDE applications are addressed in this dissertation: (1) Detection of weak $\mathrm{THz}$ pulses reflected from thick layers, and (2) Overlapping $\mathrm{THz}$ pulses reflected from thin film layers

2.2.1 Flow chart for the Matched Field Processing (MFP) algorithm. The four primary components are shown in blue. A forward model is used to sweep through a range of feasible values for an unknown parameter to generate a set of replicas that can be compared with the measured data set, using an objective function. The results of the comparison are stored in an ambiguity data set, which is searched to find an optimal value. The optimal value is the best estimate of the unknown parameter. 20

3.1.1 Sample configuration for a single layer sample in transmission mode. Top panel: A sample material with unknown $\tilde{n}_{1}$ is surrounded by an outer medium (e.g. dry air or vacuum) with $\tilde{n}_{0}$. Bottom panel: The reference measurement is made without the sample present in order to remove unknown parameters from the model. . . . . . . . . . . . . 
3.1.2 Sample configuration for a sample material of interest (e.g. paint) applied to a substrate (e.g. silicon wafer) that has known properties. Top panel: A sample material with unknown $\tilde{n}_{1}$ is applied to a substrate with known $\tilde{n}_{2}$. The bi-layer sample is surrounded by an outer medium (e.g. dry air or vacuum) with $\tilde{n}_{0}$. Bottom panel: The reference measurement is made without the sample present (i.e. substrate only) in order to remove unknown parameters from the model. . . . . . . . . .

4.1.1 Complex index of refraction for Acrylic were calculated from $\mathrm{THz}$ measurement data. Top panel: real part of the refractive index, $n$. Middle Panel: Extinction coefficient, $\kappa$. Bottom Panel: Absorption coefficient $\alpha$. Results are consistent with measurements in the literature [60]. . . 4.1.2 Left Panel. Reference waveform from $\mathrm{THz}$ reflection measurement. Right Panel. The ambiguity function (or equipment function) for the $\mathrm{THz}$ reference waveform is used to evaluate the potential performance of the maximum likelihood ratio estimator. . . . . . . . . . . . .

4.1.3 Illustration of the experiment configuration (not to scale). The numbered circles identify each of the boundaries. For each of the experiments, the spacing of the two acrylic layers was $d_{1}=d 3=5.89$ $\mathrm{mm}$ and the thickness of the lower air layer, was $d_{4}=5.62 \mathrm{~mm}$. The spacing of the air gap between the two acrylic layers was changed by inserting shims of various thickness, $d_{2}=5.62$ and $d_{2}=0.70 \mathrm{~mm}$. .

4.1.4 Comparison of results when $d_{2}=5.62 \mathrm{~mm}$. THz waveform reflected from multiple layers of planar media (top panel), the same waveform after processing with the Wiener deconvolution filter (middle panel) and after processing with the matched filter (bottom panel). The matched filter provides peaks with superior SNR. The numbers near each peak correspond to the circled boundary numbers in Figure 4.1.3. Each of the waveforms was normalized for comparison. . . . . . . . . .

4.1.5 Comparison of results when $d_{2}=0.70 \mathrm{~mm}$. THz waveform reflected from multiple layers of planar media (top panel), the same waveform after processing with the Wiener deconvolution filter (middle panel) and after processing with the matched filter (bottom panel). The matched filter provides peaks with superior SNR. The numbers near each peak correspond to the circled boundary numbers in Figure 4.1.3. Each of the waveforms was normalized for comparison. . . . . . . . . .

4.1.6 B-scan slice through the center of the unprocessed $\mathrm{THz}$ sample data illustrated in Figure 4.1.3 for the case when $d_{2}=0.70 \mathrm{~mm}$. Each column in the B-scan data is similar to the A-scan data shown in the top panel of Figure 4.1.5, and therefore the boundaries between the layers are indicated by horizontal lines in the B-scan images. . . . . . 
4.1.7 B-scan slice through the center of the sample illustrated in Figure 4.1.3 for the case when $d_{2}=0.70 \mathrm{~mm}$ from the $\mathrm{THz}$ data processed with Wiener deconvolution. Each column in the B-scan data is similar to the A-scan data shown in the middle panel of Figure 4.1.5, and therefore the boundaries between the layers are indicated by horizontal lines in the B-scan images. . . . . . . . . . . . . . . . . . .

4.1.8 B-scan slice through the center of the sample illustrated in Figure 4.1.3 for the case when $d_{2}=0.70 \mathrm{~mm}$ from the $\mathrm{THz}$ data processed with a matched filter. Each column in the B-scan data is similar to the Ascan data shown in the bottom panel of Figure 4.1.5, and therefore the boundaries between the layers are indicated by horizontal lines in the B-scan images. . . . . . . . . . . . . . . . . . . .

4.1.9 Histograms show the peak signal detection within a signal and noise time windows for the sample in Figure 4.1 .3 when $d_{2}=0.70 \mathrm{~mm}$. (Left panel.) The signal and noise histograms for the Weiner deconvolution data have a relatively wide region of overlap because the SNR in the signal time window is poor. (Right Panel.) The signal and noise histograms for the matched filter data show a smaller region of overlap because the SNR is better. Sweeping a threshold parameter across the horizontal axis of each data set produces the ROC curves in Figure 4.1.10. 67

4.1.10 ROC curves for the Wiener deconvolution and matched filter data shown in Figure 4.1.9. The curves indicate detection with the proposed matched filter method, based on the MLE, is almost ideal while the detection performance of the Wiener deconvolution method is relatively poor. . . . . . . . . . . . . . . . . .

4.1.11 ROC curves similar to those in Figure 4.1.10, show detection performance increases when the averaging per pixel is increased from 1 waveform to 10 waveforms. The performance of the Wiener deconvolution method is improved significantly by averaging, but at the expense of a 10X increase in integration time during data collection. The performance of the matched filter detector is virtually ideal. . . . . . . . .

4.2.1 Illustration of the $\mathrm{THz}$ NDE experiment configuration (not to scale). Left: Measurement configuration for the $\mathrm{THz}$ reference, which is used to approximate the THz source signal in MFP processing. Right: Measurement configuration for the layered sample under test. A calibration layer (air) with unknown thickness, $d_{0}$, accounts for the offset distance between the reference mirror and the surface of the sample. A shim (Scotch Removable Double Sided Tape) located a distance of $d_{1}$ below the sample surface creates an air gap with thickness, $d_{2}$. THz MFP is used to estimate the thicknesses of all three layers $\left(d_{0}, d_{1}\right.$, and $\left.d_{2}\right)$, simultaneously. . . . . . . . . . . . . . . 
4.2.2 Complex index of refraction for Polycarbonate were calculated from $\mathrm{THz}$ measurement data. Top panel: real part of the refractive index, $n$. Middle Panel: Extinction coefficient, $\kappa$. Bottom Panel: Absorption coefficient $\alpha$. Results are consistent with data in the literature [8], [60].

4.2.3 Measured data for Sample A. Top Panel: Mean of measured $\mathrm{THz}$ waveforms. Note that reflected THz pulse 1 (illustrated in Figure 4.2.1) arrives at approximately $15 \mathrm{ps}$, and pulses 2 and 3 overlap with one another at approximately 25 ps. Middle Panel: Spectrum of mean waveform in the top panel. Bottom Panel: Covariance matrix computed with Eq. 3.3.9 using the spectrum of each 300 measured waveform. . . . . . . . . . . . . . . . . . .

4.2.4 Measured data for Sample A. Top Panel: Mean of measured THz waveforms. Note that reflected THz pulses 1,2 and 3 (illustrated in Figure 4.2.1) all overlap on one another. Middle Panel: Spectrum of mean waveform in the top panel. Bottom Panel: Covariance matrix computed with Eq. 3.3.9 using the spectrum of each 300 measured waveform. . . . . . . . . . . . . . . . . . . .

4.2.5 Matched field ambiguity surfaces provide estimates for the thickness of the calibration layer, $d_{0}$, and polycarbonate layer, $d_{1}$, as illustrated in Figure 4.2.1 with layer thickness for Sample A given in Table 4.2.1. Top panel: Bartlett processor has a global maximum $d_{0}=190 \mu \mathrm{m}$ and $d_{1}=$ $750 \mu \mathrm{m}$. Bottom panel: MV processor has a global maximum $d_{0}=$ $180 \mu \mathrm{m}$ and $d_{1}=750 \mu \mathrm{m}$. The results for $d_{1}$ are consistent with ground truth measurements with a Vernier caliper. Ground truth data is not available for the calibration layer, but the these results are reasonable, and consistent between both Bartlett and MV processors. See Table 4.2.2 and Figure 4.2.8 for a comparison of measurement errors for layers in all samples. . . . . . . . . . . . . . . . . . .

4.2.6 Matched field ambiguity surfaces provide estimates for the thickness of the polycarbonate layer, $d_{1}$ and the air gap, $d_{2}$, as illustrated in Figure 4.2.1 with layer thickness for Sample A given in Table 4.2.1. Top panel: Bartlett processor has a global maximum $d_{1}=750 \mu \mathrm{m}$ and $d_{2}=70 \mu \mathrm{m}$. Bottom panel: MV processor has a global maximum $d_{1}=750 \mu \mathrm{m}$ and $d_{2}=70 \mu \mathrm{m}$. These results are consistent with ground truth measurements with a Vernier caliper. See Table 4.2.2 and Figure 4.2.8 for a comparison of measurement errors for layers in all samples. 
4.2.7 Matched field ambiguity surfaces provide estimates for the thickness of the polycarbonate layer, $d_{1}$ and the air gap, $d_{2}$, as illustrated in Figure 4.2.1 with layer thickness for Sample D given in Table 4.2.1. Top panel: Bartlett processor has a global maximum $d_{1}=250 \mu \mathrm{m}$ and $d_{2}=60 \mu \mathrm{m}$. Bottom panel: MV processor has a global maximum $d_{1}=240 \mu \mathrm{m}$ and $d_{2}=60 \mu \mathrm{m}$. These results are consistent with ground truth measurements with a Vernier caliper. See Table 4.2.2 and Figure 4.2.8 for a comparison of measurement errors for layers in all samples.

4.2.8 For each of the Vernier caliper measurements listed in Table 4.2.2, the corresponding thickness estimates from THz MFP with the Bartlett and MV processors are plotted as blue circles and red squares, respectively. The dashed line represents an ideal case of equality of the Vernier caliper measurement and the THz MFP thickness estimate. Thus, all of the measurement data from the $\mathrm{THz} \mathrm{MFP}$ approach is in close agreement with the Vernier caliper data. . . . . . . . . . . . . . .

4.3.1 Illustration of the $\mathrm{THz}$ NDE configuration for paint film experiments (not to scale). Left: Measurement configuration for the $\mathrm{THz}$ reference, which is used to approximate the $\mathrm{THz}$ source signal in MFP processing. Right: Measurement configuration for the layered sample under test. A calibration layer (air) with unknown thickness, $d_{0}$, accounts for the offset distance between the reference mirror and the surface of the sample. Here, the paint layer thickness is denoted as $d_{1}$. THz MFP is used to estimate the thicknesses of both layers $\left(d_{0}\right.$ and $\left.d_{1}\right)$, simultaneously.

4.3.2 Dupli-Color@Perfect Match@paint is an acrylic lacquer aerosol paint designed to duplicate original General Motors (GM) colors [61]. . . . .

4.3.3 Six (6) different paint samples (A - F) were created by spray painting aluminum plates with Dupli-Color@Perfect Match@paint. Sample A was created by applying several coats of spay paint to make the sample with the thickest layer. Fewer coats were applied to the subsequent

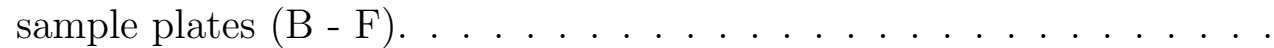

4.3.4 Photo of the CEM DT-156 coating thickness tester, which uses eddy currents induced in the aluminum substrate to measure coating thicknesses [62]. The electromagnetic sensor on the bottom of the instrument must make direct contact with paint to make a measurement. The instrument is calibrated to account for dielectric coatings between the instrument's sensor and the aluminum substrate and reports coating thickness measurements in microns. . . . . . . . . . . . . . . .

4.3.5 Part of a silicon wafer was coated with a layer of Dupli-Color@Perfect Match@paint. This sample was used in a separate $\mathrm{THz}$ spectroscopy experiment to extract the index of refraction, which is needed as an input when generating replicas to be used for THz MFP. . . . . . . . . 
4.3.6 Complex index of refraction for the red acrylic paint were calculated from $\mathrm{THz}$ transmission measurements with the silicon wafer sample shown in Figure 4.3.5. Top panel: real part of the refractive index, $n$.

Bottom Panel: Extinction coefficient, $\kappa . \ldots \ldots \ldots \ldots$

4.3.7 Sample A Measurement data Top Panel: Mean of measured THz waveforms. Middle Panel: Spectrum of mean waveform in the top panel. Bottom Panel: Covariance matrix computed with Eq. 3.3.9 using the spectrum of each of the 300 measured waveforms. . . . .

4.3.8 Sample A: Matched field ambiguity surfaces provide estimates for the thickness of the calibration layer, $d_{0}$, and acrylic paint layer, $d_{1}$. Top panel: Bartlett processor global maximum at $d_{0}=198 \mu \mathrm{m}$ and $d_{1}=112 \mu \mathrm{m}$. Bottom panel: MV processor global maximum at $d_{0}=196 \mu m$ and $d_{1}=112 \mu m \ldots \ldots \ldots \ldots \ldots$

4.3.9 Sample B: Matched field ambiguity surfaces provide estimates for the thickness of the calibration layer, $d_{0}$, and acrylic paint layer, $d_{1}$. Top panel: Bartlett processor global maximum at $d_{0}=248 \mu \mathrm{m}$ and $d_{1}=$ $74 \mu \mathrm{m}$. Bottom panel: MV processor global maximum at $d_{0}=246 \mu \mathrm{m}$ and $d_{1}=74 \mu m . \ldots \ldots \ldots \ldots \ldots \ldots \ldots \ldots \ldots \ldots \ldots$

4.3.10 Sample C: Matched field ambiguity surfaces provide estimates for the thickness of the calibration layer, $d_{0}$, and acrylic paint layer, $d_{1}$. Top panel: Bartlett processor global maximum at $d_{0}=272 \mu \mathrm{m}$ and $d_{1}=$ $50 \mu \mathrm{m}$. Bottom panel: MV processor global maximum at $d_{0}=272 \mu \mathrm{m}$ and $d_{1}=50 \mu m \ldots \ldots \ldots \ldots \ldots \ldots \ldots$

4.3.11 Sample D: Matched field ambiguity surfaces provide estimates for the thickness of the calibration layer, $d_{0}$, and acrylic paint layer, $d_{1}$. Top panel: Bartlett processor global maximum at $d_{0}=292 \mu \mathrm{m}$ and $d_{1}=$ $26 \mu \mathrm{m}$. Bottom panel: MV processor global maximum at $d_{0}=292 \mu \mathrm{m}$ and $d_{1}=26 \mu m \ldots \ldots \ldots \ldots \ldots \ldots \ldots \ldots \ldots \ldots$

4.3.12 Sample E: Matched field ambiguity surfaces provide estimates for the thickness of the calibration layer, $d_{0}$, and acrylic paint layer, $d_{1}$. Top panel: Bartlett processor global maximum at $d_{0}=288 \mu \mathrm{m}$ and $d_{1}=$ $30 \mu \mathrm{m}$. Bottom panel: MV processor global maximum at $d_{0}=286 \mu \mathrm{m}$ and $d_{1}=32 \mu m \ldots \ldots \ldots \ldots \ldots \ldots$

4.3.13 Sample F: Matched field ambiguity surfaces provide estimates for the thickness of the calibration layer, $d_{0}$, and acrylic paint layer, $d_{1}$. Top panel: Bartlett processor global maximum at $d_{0}=298 \mu \mathrm{m}$ and $d_{1}=$ $20 \mu \mathrm{m}$. Bottom panel: MV processor global maximum at $d_{0}=308 \mu \mathrm{m}$ and $d_{1}=12 \mu \mathrm{m}$. 
4.3.14 For each of the CEM DT-156 Coating Thickness Tester measurements listed in Table 4.3.2, the corresponding thickness estimates from $\mathrm{THz}$ MFP with the Bartlett and MV processors are plotted as blue circles and red squares, respectively. The dashed line represents an ideal case of equality of the Coating Thickness Tester measurement and the $\mathrm{THz}$ MFP thickness estimate. Thus, most of the measurement data from the $\mathrm{THz}$ MFP approach is in close agreement conventional measurements, except for small errors for the thinnest sample (sample F). . . . . . . 113 


\section{Chapter 1}

\section{Introduction and Motivation}

\subsection{Introduction}

During the 20th century many technological advances were made using electromagnetic waves in both the microwave and optical regions of the spectrum. However, the band of frequencies between these two regions, referred to as the terahertz gap [1], had been difficult to access due to the technical challenges of generating and detecting waves at the far edges of the microwave and optical portions of the spectrum. Figure 1.1.1 shows the location of the terahertz gap in the electromagnetic spectrum.

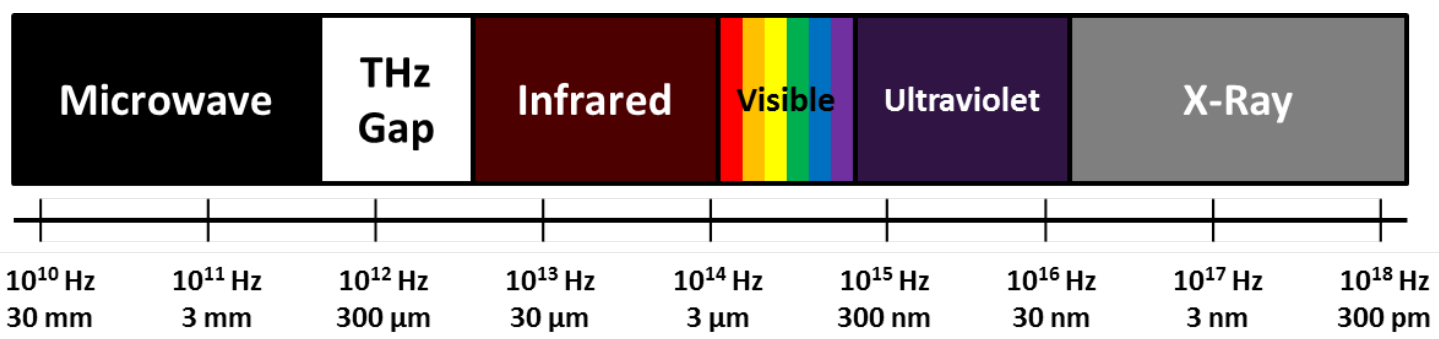

Figure 1.1.1: The terahertz gap is a portion of the electromagnetic spectrum between microwave (radio) and optical frequencies. Electronics and photonics technologies have developed on either side of this gap, but were not able to reach into this region of the spectrum until recently. 
In the 1990's advances in laser technology and semiconductor technology lead to the development of the Austin switch. This innovation, combined with other technological advances in broadband antennas, lithography, computer controls and digital signal processing, provided a method generating and measuring pulses with duration on the order of a few picoseconds. Thus, these new systems are capable of operating over incredibly wide bandwidths, typically $0.1-3.0 \mathrm{THz}$.

\subsection{Pulsed THz Systems}

A variety of methods for generating and detecting $\mathrm{THz}$ waves have been explored, and a thorough discussion of the history of development can be found in the literature $[2],[3]$. One of the key development in terahertz systems occurred when the modelocked Ti:saphire laser became commonly available in the 1980's, and it is the cost of the laser which still accounts for most of the total cost of $\mathrm{THz}$ spectroscopy systems today [3]. These lasers have pulse durations below 100 femtoseconds $\left(10^{-15}\right)$, which are directed with mirrors to special semiconductors or crystals where they stimulate a secondary pulse of electric current. The resulting current pulses have a slightly longer duration on the order of picoseconds $\left(10^{-12}\right)$ with bandwidths extending up to terahertz $\left(10^{12}\right)$ frequencies.

One of the primary types of pulsed $\mathrm{THz}$ emitters used today are photo-conducting antennas (PCA's). The first PCA was developed in 1981, and was applied to THz TDS systems in 1988-89 [3]. Optimization of the substrate, antenna and lens design has improved the overall efficiency and increased output power from nano-watts to micro-watts with bandwidths extending from $100 \mathrm{GHz}$ to $2 \mathrm{THz}$ [3]. Typical laser power is on the order of 1 Watt [3], and result in THz pulses of approximately 30-40 
micro-watts [3], although some systems have reached 100 micro-watts [4]. Systems using optical rectification were developed in 1995 and have wider bandwidth, but lower output power [3].

Figure 1.2.1 shows a photo of a pulsed $\mathrm{THz}$ system that was commercially developed and manufactured by Picometrix Inc. This design is used in the T-Ray $4000 \mathrm{THz}$ system in the Northwest Electromagnetics and Acoustics Research (NEAR) Lab at Portland State University (PSU), and is similar to other pulsed THz systems that are currently used for research. A femtosecond laser, beam splitter and mirrors are fully enclosed inside the main control module. Fiberoptic and power cables connect the T-Ray 4000 to transmit (Tx) and receive ( $\mathrm{Rx}$ ) heads which are mounted securely on an optical lab table. Femtosecond laser pulses are guided by the fiberoptic cables and directed to both the $\mathrm{Tx}$ and $\mathrm{Rx}$ head. Inside the $\mathrm{Tx}$ head the laser pulse interacts with a PCA to generate a $\mathrm{THz}$ pulse. The $\mathrm{THz}$ pulse is transmitted through the sample and is detected coherently using another PCA inside the Rx head. 


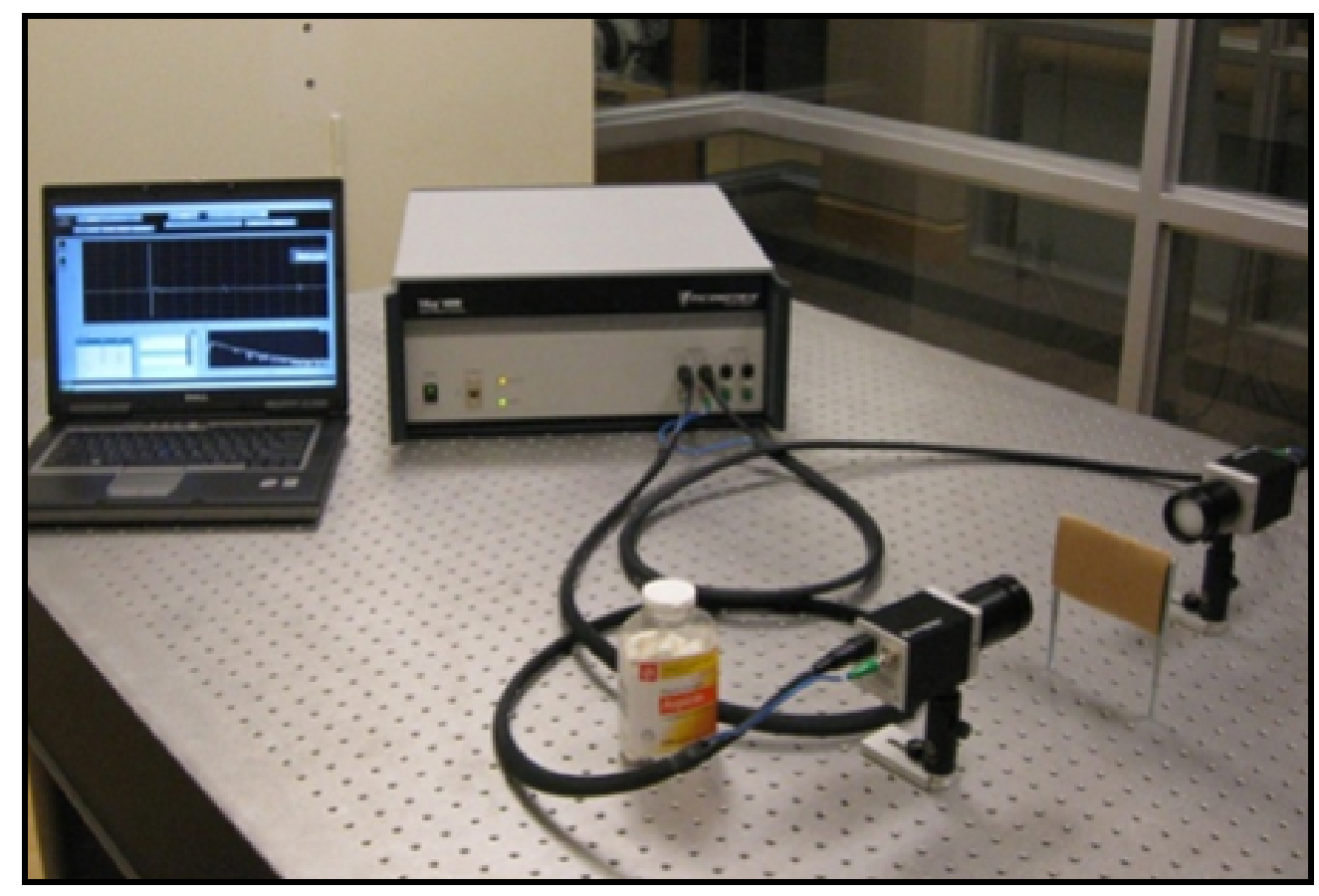

Figure 1.2.1: The T-Ray 4000 is used for THz NDE research in the Northwest Electromagnetics and Acoustics Research (NEAR) Lab at Portland State University (PSU). It was developed and manufactured by Picometrix Inc.

The receiver consists of a single photo-conductive antenna sensor fed by a separate laser probe path that includes a variable delay line to record the $\mathrm{THz}$ pulse waveform. Since the duration of the laser pulse is much shorter than the $\mathrm{THz}$ pulse, only a sample of the total $\mathrm{THz}$ pulse is captured for each laser pulse. Coherent detection is accomplished by changing the relative time delay between the probe and pump paths until a complete $\mathrm{THz}$ pulse waveform is recorded. The exact relationship between the current and $\mathrm{THz}$ field is often unknown, and measurements are typically recorded in arbitrary units (a.u.). A detailed explanation of $\mathrm{THz}$ measurement systems can be found in the literature [2], [3].

Figure $1.2 .2 \mathrm{a}$ shows a typical $\mathrm{THz}$ pulse waveform detected by a Picometrix T-Ray 4000 spectroscopy system. After performing a Fourier transform, the power spectral 


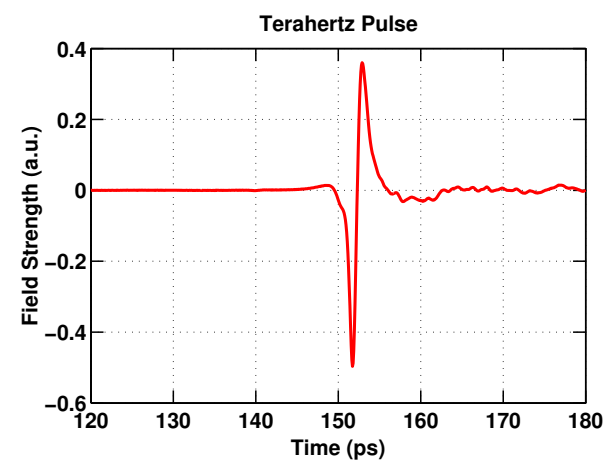

(a)

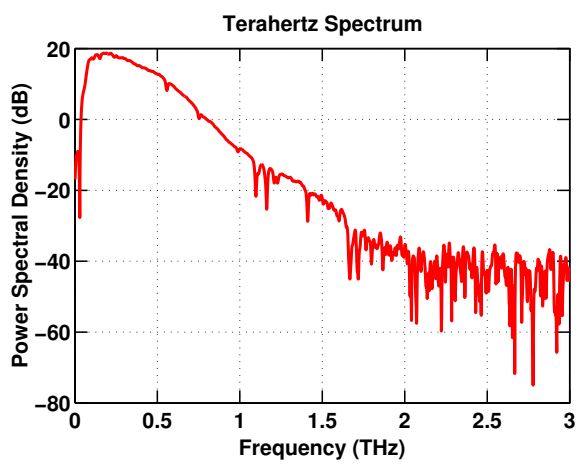

(b)

Figure 1.2.2: (a) A THz Pulse measured with the Picometrix T-Ray 4000 at the NEAR-Lab. The pulse duration is approximately 5 picoseconds (ps). (b) The power spectral density (PSD) of the pulse shown in figure 1.2.2a. The usable portion of the spectrum spans approximately from 0.1 to $2 \mathrm{THz}$.

density (PSD) can be plotted as shown in Figure 1.2.2b.

Modern $\mathrm{THz}$ systems have been able to achieve bandwidths spanning almost five octaves $(0.1-3.0 \mathrm{THz})$ and important material properties in this band have been discovered. Polar molecules have charge imbalances which give rise to unique rotational and vibrational resonances at $\mathrm{THz}$ frequencies [3]. These resonances cause the overall permittivity of the material to vary with frequency in a dramatic but predictable manner, providing a unique spectroscopic signature or fingerprint. For example, the spectral signatures of various explosives and drugs are available in the literature [5], [6]. Non-polar molecules (commonly found in packaging materials) have no resonances in the $\mathrm{THz}$ band and are often transparent to $\mathrm{THz}$ frequencies [7].

The following section provides a brief overview of the potential applications for $\mathrm{THz}$ systems, the challenges encountered when attempting to implement $\mathrm{THz}$ systems for real-world applications, and the approach that is proposed in this research. 
Table 1.3.1: THz NDE offers advantages over conventional methods.

\begin{tabular}{|c|c|c|c|c|c|}
\hline $\begin{array}{c}\text { Imaging } \\
\text { Modalities }\end{array}$ & $\begin{array}{c}\text { Non- } \\
\text { contact }\end{array}$ & $\begin{array}{c}\text { Safe for } \\
\text { Human Exposure }\end{array}$ & $\begin{array}{c}\text { Sensitive } \\
\text { to Dielectrics }\end{array}$ & $\begin{array}{c}\text { Sensitive } \\
\text { to Metal }\end{array}$ & $\begin{array}{c}\text { Chemical } \\
\text { Mapping }\end{array}$ \\
\hline Terahertz & $\checkmark$ & $\checkmark$ & $\checkmark$ & $\checkmark$ & $\checkmark$ \\
\hline X-rays & $\checkmark$ & & & $\checkmark$ & \\
\hline Ultrasound & & $\checkmark$ & $\checkmark$ & $\checkmark$ & \\
\hline Eddy Current & & $\checkmark$ & $\checkmark$ & $\checkmark$ & \\
\hline
\end{tabular}

\subsection{Potential Applications for Terahertz Technology}

THz systems offer a unique combination of capabilities with the potential for many exciting new applications in the future. Many packaging materials (e.g. polymers, paper, cardboard, cloth, etc.) are non-polar with relatively low loss in the $\mathrm{THz}$ spectrum. The wide bandwidth of $\mathrm{THz}$ systems allows for fairly detailed imaging of objects with dimensions on the order of hundreds of microns. In addition, many materials of interest, such as drugs, explosives, etc. are composed of polar materials with absorption peaks in the THz spectrum. Thus, $\mathrm{THz}$ waves can be used for NDE and chemical mapping of the internal components of sample materials.

Table 1.3.1 shows that $\mathrm{THz}$ systems offer several advantages over more conventional NDE techniques, such as X-rays and ultrasound. One of the greatest benefits of $\mathrm{THz}$ sensing is the ability to make non-contact measurements of dielectric media, which previously could only be measured by destructive evaluation of representative samples, or with other sensors that require contact with the surface of the sample such as ultrasound or magnetic sensors. Although X-rays can be non-contact and non-destructive to the sample, they can be harmful to humans performing the testing and are not always capable of revealing contrast between dielectric materials. $\mathrm{THz}$ 
waves have the advantage of revealing spectroscopic that cannot be observed with $\mathrm{X}$-rays and since $\mathrm{THz}$ waves are non-ionizing they are considered safe for human exposure at the power levels currently used in most TDS systems. In addition, the size and cost of broadband $\mathrm{THz}$ sensors has reduced significantly since they were first introduced for laboratory research in the 1990s.

THz Time Domain Spectroscopy (TDS) is a promising technology with a broad range of potential applications in fields such as medical imaging, security screening and nondestructive evaluation (NDE) [4], [8]. THz NDE applications include investigation of products consisting of layered media such as pharmaceutical tablets [9]-[11], automobile paint [8], [12]-[16], microelectronic circuits [17] and many other industrial products manufactured under well-controlled conditions [8], [18]. Similar NDE methods may be used in more complex scenarios for security screening of packages and luggage for detection of threat materials [1], [5], investigation of historical artifacts such as paint layers in artwork [19]-[23] or written manuscripts which are too brittle to be opened [21], [24]. In addition, the ability of $\mathrm{THz}$ reflection spectroscopy to measure the water content within layers is important for the detection of skin cancer [25], [26] and the hydration of plant tissues [18], [27].

\subsection{Challenges in Terahertz Non-Destructive Evaluation}

Many of the proposed $\mathrm{THz}$ NDE applications require both fast scanning rates (low integration time) and algorithms to automatically detect $\mathrm{THz}$ pulses in the reflected or transmitted $\mathrm{THz}$ pulse trains. These $\mathrm{THz}$ pulses arise from changes in refractive index at the layer boundaries, while the time delay between pulses is governed by the group velocity of the THz pulses in the media. Thus, the information contained 
within the measured $\mathrm{THz}$ waveform is useful for the detection and localization of subsurface object boundaries and/or defects inside the media.

The research presented here addresses problems that arise when incorporating pulsed $\mathrm{THz}$ systems for NDE of layered media. Figure 1.4.1 illustrates the two primary challenges for THz NDE applications that are addressed in this dissertation: (1) Detection of weak $\mathrm{THz}$ pulses reflected from thick layers, and (2) Overlapping $\mathrm{THz}$ pulses reflected from thin film layers

\section{Thick layers}
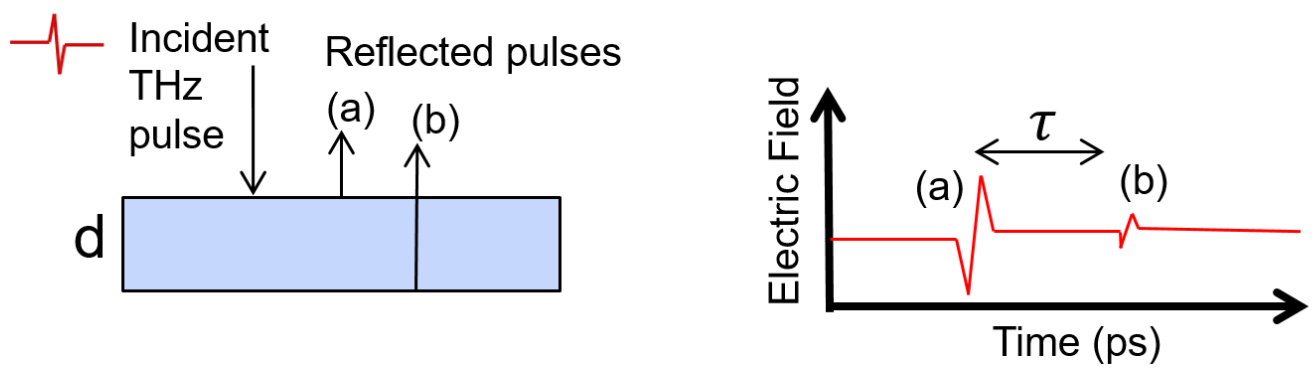

\section{Thin films}
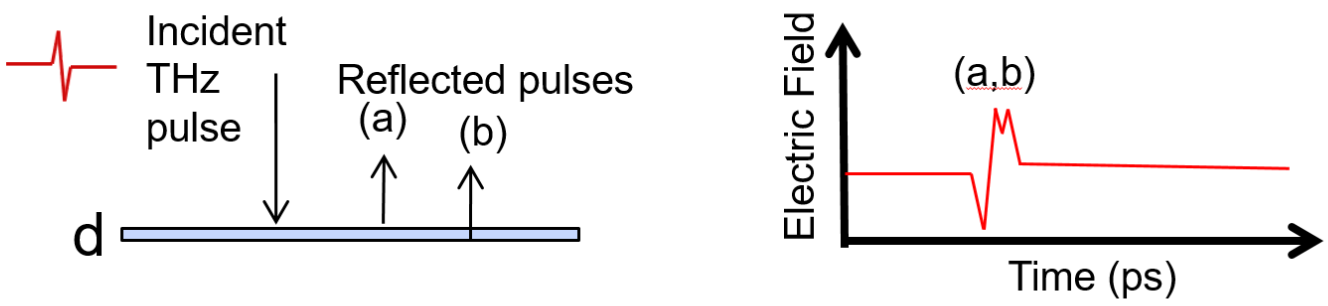

Figure 1.4.1: Two primary challenges for THz NDE applications are addressed in this dissertation: (1) Detection of weak $\mathrm{THz}$ pulses reflected from thick layers, and (2) Overlapping $\mathrm{THz}$ pulses reflected from thin film layers 


\subsubsection{Low SNR in Fast $\mathrm{THz}$ Scans of Thick Layers}

Fast scanning of samples is desirable for many NDE applications in industrial quality control or bio-medical screening. However, $\mathrm{THz}$ pulses reflecting from layer interfaces are often obscured by noise when the THz scans are performed with minimal integration time. $\mathrm{THz}$ pulses that travel through thick layers with high absorption will have relatively low signal to noise ratios (SNR), which can be reduced further if crossing multiple layer boundaries.

Early THz researchers attempted to identify material boundaries by direct interpretation of the raw $\mathrm{THz}$ waveforms. However, the identification of the $\mathrm{THz}$ pulses is often complicated by oscillations in the envelope of the $\mathrm{THz}$ source pulse. Others have used deconvolution processing to approximate the impulse response for the sample under test. Often a Wiener filter is used to make the echo pulses in the signal waveform

more evident [1], but in low SNR conditions the results are often subject to human interpretation and confirmation bias. A more robust processing approach is needed in low SNR conditions.

\subsubsection{Overlapping THz Pulses in Thin Films}

In thin film samples, the time-of-flight within a layer is less than the duration of the $\mathrm{THz}$ pulse and the $\mathrm{THz}$ pulses reflecting from the boundaries overlap on top of one another. Consequently it is not possible to use conventional time domain reflectometry (TDR) techniques to estimate the thickness of thin films.

Others have been able to estimate the thickness of thin films with model-based param- 
eter estimation techniques [12], [13], [28]-[30]. However, much of the initial research has focused primarily on measurement and modeling techniques, with relatively little attention given to the processing methodology or cost functions used to compare the modeled and measured data. There is a need to incorporate more advanced physicsbased signal processing techniques.

\subsection{Physics-Based Signal Processing Approach}

Although modern $\mathrm{THz}$ measurement systems are capable of scanning dielectric sample materials with $\mathrm{THz}$ transmitters and receivers oriented in a variety of configurations, they often lack the advanced physics-based signal processing algorithms needed for NDE applications. In this dissertation physics-based signal processing methods that been historically used for radar/sonar signal processing are adapted and applied to detect boundaries in THz NDE data.

\subsubsection{Derivation of the Matched Filter for Low-SNR Applications}

For many THz NDE applications, the sample and measurement system are both stationary and the objective is to detect only the boundary between the layers of stratified media using a $\mathrm{THz}$ transmitter and receiver with either a monostatic or bi-static measurement geometry. Others have used deconvolution processing with a Weiner filter or other inverse filters to get an approximation of the impulse response [8]. However, this approach is not well suited to low SNR conditions, which are typically encountered with low signal integration time, i.e. applications that require fast scanning. 
The method introduced here uses the maximum likelihood estimator (MLE) for the detection of layer boundaries in THz NDE applications. This approach is well known in radar/sonar signal processing literature [31]-[33], but to the author's knowledge this is the first time it has been implemented for THz NDE. Thus, the objective of boundary detection in THz NDE is framed in terms of statistical signal processing, in which the goal is to use the $\mathrm{THz}$ source signal and the signal measured from a sample to estimate a parameter, i.e. time delay. The parameter values (delays) that maximize the output of the MLE are then used to identify reflected $\mathrm{THz}$ pulses in the received $\mathrm{THz}$ pulse train. Then the time difference of arrival (TDOA) between the detections can be used along with the index of refraction (assumed to be known a priori) to calculate the distance traveled, i.e. the thickness of the layer.

It is shown that for pulsed THz systems, the source signal may be estimated by the reference waveform, and that the $\mathrm{THz}$ signal measured with the layered sample can be modeled as a modified form of the source waveform that has been delayed and attenuated by propagation through the sample media along with the addition of white noise. Thus, using an analytic signal model, the MLE is shown to reduce to the matched filter, which appears often in radar/sonar signal processing applications.

The performance of the matched filter vs. the conventional Wiener deconvolution approach is evaluated for the detection of $\mathrm{THz}$ pulse reflections (i.e. layer boundaries) with $\mathrm{THz}$ signals that were measured with minimal signal integration time. Histograms of the detection results are presented and discussed and Receiver Operating Characteristic (ROC) curves are used to show the matched filter performs better for signals collected in low SNR conditions. This is explained in terms of the mathematical formulations of the Wiener filter and matched filter. 
Furthermore, it is shown that the performance limitations of the matched filter (i.e. thickness resolution limit) can be characterized by the ambiguity function. It is shown that width of the main peak of the ambiguity function provides an estimate of minimum resolution for layer thickness that agrees with measured results. Layers that are thinner than the resolution limit are considered to be "thin films".

\subsubsection{Adaptation of Matched Field Processing Techniques for Thin Films}

In this dissertation, the problem of measuring thin film thickness is addressed using a model-based parameter estimation approach found primarily in the underwater ocean acoustics literature. Matched field processing (MFP) is a robust mathematical framework that has been developed over several decades for sonar signal processing.

Historically, MFP has been used for source localization (in depth and range) when simple plane wave beamforming techniques could not be account for complicated multipath propagation in the acoustic waveguide formed by the sea surface and seafloor. Typically, the environmental parameters are measured independently or assumed to be known and the goal of MFP is to use data recorded with an array of hydrophone receivers to estimate the position of the sound source (i.e. its range, depth and sometimes azimuth), relative to the receiver position. A logical extension for these techniques has been to record acoustic data with a known sound source position and estimate the environmental parameters between the source and retriever(s). The use of MFP for estimation of environmental properties is sometimes referred to as matched field tomography in the literature [34], [35].

MFP typically exploits only the spatial coherence of the field as measured by an 
array of sensors after performing a Discrete Fourier Transform (DFT) on the data measured by each sensor. However, when the source is coherent across a broad band of frequencies, then the temporal coherence may also be exploited [36]. Thus, it is possible that the temporal coherence of the THz TDS spectrum could be exploited in a MFP algorithm to extract thickness information from thin film materials using only a single $\mathrm{THz}$ sensor.

The MFP approach typically compares the modeled "replica" spectra with the sample covariance of the measured spectrum. The two most popular cost functions (or "processors") used in the MFP literature are the Bartlett processor and the Minimum Variance (MV) processor. Often a global search of the parameter space is performed and results are displayed as two-dimensional (2D) ambiguity surfaces. The peak of the ambiguity surface identifies the best estimate of the unknown parameter(s).

After reviewing the MFP literature the author developed a similar approach for $\mathrm{THz}$ NDE of layered media, which can be applied to both thick layers and thin films. A transfer matrix model was developed to model the transfer functions for plane wave propagation within layered media samples. The resulting transfer functions were convolved with the $\mathrm{THz}$ source spectrum, which is approximated from the $\mathrm{THz}$ reference pulse to produce replicas of the received spectra for various layer thicknesses. The replica spectra are compared with the sample covariance of the measured spectra using both the Bartlett and MV processors. Finally, the results are organized to plot 2D ambiguity surfaces. The modeling parameters (layer thicknesses) corresponding to the peaks of the Bartlett and MV ambiguity surfaces provide an estimates of the layer thickness, which agrees well with independent measurements made with other laboratory instruments. 
To the author's knowledge this is the first time that MFP methods using the covariance matrix, the Bartlett processor, the Minimum variance processor and ambiguity plots have been applied to $\mathrm{THz}$ NDE. Thus, new and novel methods for THz NDE are introduced.

\subsection{Contributions and Significance of this Work}

This research proposes several physics-based signal processing methods from radar and sonar signal processing applications to address the challenges encountered in $\mathrm{THz}$ NDE of layered media. Results are demonstrated with measured data from a pulsed THz system in the Northwest Electromagnetic and Acoustics Research Laboratory (NEAR-Lab) at Portland State University (PSU). It is expected that this research will provide an important link for $\mathrm{THz}$ researchers to access and apply the robust methods available in the MFP literature.

The key contributions of this work are:

1. Development of a matched filter approach for $\mathrm{THz} \mathrm{NDE}$ of thick layered media measured in low SNR conditions

- Trade-offs between processing THz A-scans with a matched filter and conventional Wiener filter techniques are demonstrated.

- Receiver Operating Characteristic (ROC) curves are used to show the matched filter provides superior performance in low SNR conditions.

- Results were presented in at the International Symposium on Optomechatronic Technologies (ISOT) conference in Seattle, WA in November 2014 
and published in the conference proceedings [37].

2. Development of a new MFP algorithm for $\mathrm{THz}$ NDE of thin-film layered media, based on techniques in the underwater acoustics literature.

- Accurate thickness measurements of thin film polymer materials on the order of 10's of microns is demonstrated.

- Simultaneous estimation of multi-layer films is demonstrated.

- Results were published as a peer-reviewed journal publication in the Sensors Journal special issue on THz sensors in October 2018 [38].

- To the author's knowledge this is the first time that MFP concepts, such as use of the sample covariance matrix, Bartlett processor and MV processor have appeared in the $\mathrm{THz}$ NDE literature. 


\section{Chapter 2}

\section{Literature Review}

This chapter provides a brief literature review of the state of art for $\mathrm{THz}$ NDE of layered media. A brief introduction to MFP is also discussed with references to underwater acoustics literature.

\section{1 $\mathrm{THz}$ Non-Destructive Evaluation of Layered Media}

The ability of terahertz $(\mathrm{THz})$ waves to penetrate dielectric materials and detect material boundaries has generated great interest in $\mathrm{THz}$ research for non-destructive evaluation (NDE) applications such as pharmaceutical tablets, automobile paint, and many other industrial products manufactured under well-controlled conditions [4], [8]. Many of these applications require both fast scanning rates (low integration time) and algorithms to automatically detect echo pulses in the reflected or transmitted $\mathrm{THz}$ waveforms. These echo pulses arise from changes in refractive index at the boundaries of an object and its layers, while the time delays are governed by the group velocity of the $\mathrm{THz}$ pulses in the media. Thus, the information contained within the $\mathrm{THz}$ waveform is useful for the detection and localization of subsurface object boundaries 
and/or defects inside the media. However, identification of the $\mathrm{THz}$ echo pulses is complicated by oscillations in the envelope of the THz source pulse. Furthermore, $\mathrm{THz}$ echo pulses are often obscured by noise when scans are performed with minimal integration time.

In a majority of $\mathrm{THz} \mathrm{NDE}$ applications, the refractive indices of the materials involved are known, as is the order in which the layers appear. Since the bandwidth of $\mathrm{THz}$ pulses is relatively wide (typically $0.1-3.0 \mathrm{THz}$ ) dispersion occurs as the $\mathrm{THz}$ pulse passes through most materials of interest. Therefore, researchers typically use the group velocity (obtained from spectroscopic analysis of transmission data from thin samples) and the time delay between reflected $\mathrm{THz}$ pulses (visible in A-scans from a layered media) to calculate the layer thickness. Defects such as air bubbles within the media may be identified as unexpected peaks that appear in the A-scan waveforms or B-scan images.

Early $\mathrm{THz}$ researchers attempted to identify material boundaries by direct interpretation of the raw THz waveforms. THz NDE methods have typically relied on manual evaluation and somewhat subjective human interpretation to locate layer boundaries or defects from a train of echo pulses in an A-scan [8], [12]-[14], [16], [25], [39]-[42]. This is because the THz pulse typically contains at least one zero-crossing between the negative and positive portions of the $\mathrm{THz}$ pulse. In addition, the reflected pulses may be inverted depending on the difference between the refractive indices (i.e. impedance mismatches) at the layer boundaries. Finally, transmission through lossy media often results in relatively low signal-to-noise ratios (SNR) making interpretation of the results a subjective process that is prone to confirmation bias.

Others have adapted relatively complicated signal processing techniques to make 
the reflected THz pulses in the signal waveform more evident [8], but the results are still subject to human interpretation and confirmation bias. Some scientific researchers have made improvements to $\mathrm{THz}$ NDE processing by applying deconvolution (a method used in THz spectroscopic analysis [3]) in an attempt to convert the $\mathrm{THz}$ single-cylcle pulse to an approximate impulse. However, the pulses resulting from deconvolution often contain significant ringing adjacent to the main peak. These smaller peaks make it difficult to interpret pulse trains, especially with low SNR. These spurious peaks can easily be mistaken for additional boundaries or defects in the media. Consequently, researchers have made further attempts to reduce the ringing that appears in the deconvolved waveforms using various inverse filtering techniques [8], [25], [40]-[42]. While such efforts have been helpful in advancing THz NDE, the techniques can be difficult to apply in general. Designing and optimizing a filter that attenuates the anomalous peaks while preserving the main peak can be time-consuming and may not be entirely successful at removing all of the ringing artifacts.

In [37] the author used a matched filter to process THz NDE data based on techniques that have been used to estimate the range to targets in radar and active sonar applications. Approaching THz NDE from this standpoint makes it possible to leverage some relatively mature statistical signal processing methods, and facilitates the automation of THz NDE processing using unbiased thresholds instead of human interpretation. For example, detection performance can be explored using classic methods such as receiver operating characteristic (ROC) curves [37]. Using samples constructed of layers of polymer materials, it was shown that the output of the matched filter has better SNR than deconvolution while providing similar range resolution [37].

In recent years $\mathrm{THz}$ researchers have attempted to measure the thickness of thin 
films for important NDE applications that include the measurement of paint film thicknesses and layers within laminated media. In such applications the $\mathrm{THz}$ pulses reflecting from the front and back interfaces of the layer overlap with one another and conventional time domain techniques cannot be used to estimate the thickness.

One approach to estimate the thickness of thin films that has emerged in the literature is to minimize the MSE between a measured THz waveform (composed of overlapping $\mathrm{THz}$ pulses from the front/back boundaries) and a set of simulated waveforms that result from films of known material properties and known thickness [12], [13], [28][30].

\subsection{Matched Field Processing}

MFP is an indirect measurement technique in which the temporal and spatial coherence of the field may be used to match experimental data with a corresponding set of data (often called "replicas" in the literature) that are generated by simulating the experiment with a physical model. One or more variables in the physical model may be used as the sweep parameter when creating the replica set [34], [35]. Then, an objection function is used to compare the measured data set with the replica sets generated by the model using a range of feasible values for the sweep parameter. Finally, the parameter that gives the optimum output from the objective function is identified as the best estimate of the measured variable. Figure 2.2.1 shows a diagram that illustrates the MFP algorithm. 

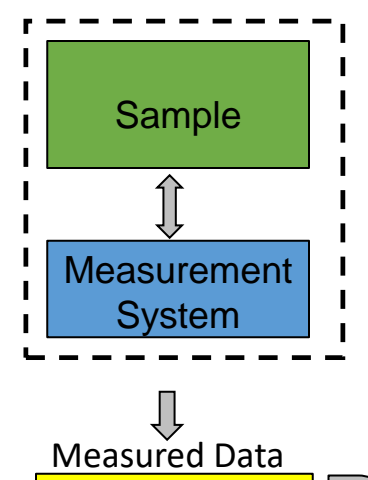

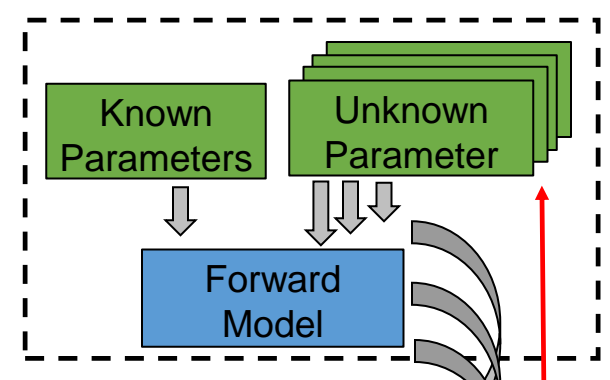

Replica Data

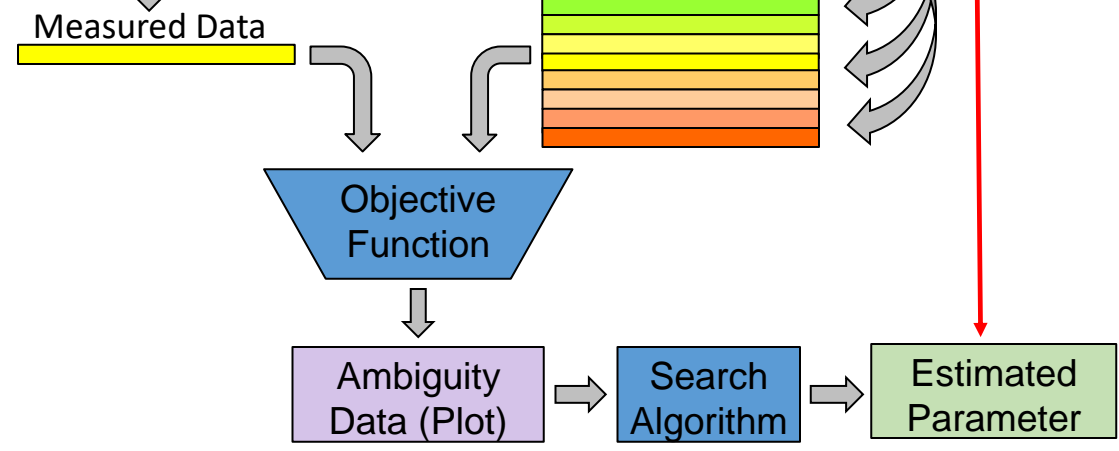

Figure 2.2.1: Flow chart for the Matched Field Processing (MFP) algorithm. The four primary components are shown in blue. A forward model is used to sweep through a range of feasible values for an unknown parameter to generate a set of replicas that can be compared with the measured data set, using an objective function. The results of the comparison are stored in an ambiguity data set, which is searched to find an optimal value. The optimal value is the best estimate of the unknown parameter.

Thus, MFP consists of essentially four elements [43]:

1. a measurement system that is used to record a data set,

2. a propagation model that is used to generate a replica data set by sweeping through a range of feasible values for some unknown parameter(s), 


\section{3. an objective function relating the measured and modeled data, and}

\section{4. an efficient algorithm for searching the parameter space.}

Historically, MFP has been used for source localization (in depth and range) when simple plane wave beamforming techniques could not be account for complicated multipath propagation in the acoustic waveguide formed by the sea surface and seafloor. Typically, the environmental parameters are measured independently or assumed to be known and the goal of MFP is to use data recorded with an array of hydrophone receivers to estimate the position of the sound source (i.e. its range, depth and sometimes azimuth), relative to the receiver position [34], [35].

A logical extension for these techniques has been to record acoustic data with a known sound source position and estimate the environmental parameters between the source and retriever(s). The use of MFP for estimation of environmental properties is sometimes referred to as matched field tomography in the literature [34], [35]. One important example of matched field tomography is the estimation of geo-acoustic properties of the sea floor, including thickness of sediment layers as well as the sound speed and attenuation within each layer. In the late 1990's, MFP was applied to

electromagnetics for the radar systems locating airborne vehicles within the layers of the troposphere [43].

In most of the MFP literature, the modeled and measured data is generally converted to discrete time bins with a discrete Fourier transform for an array of sensors (and sometimes a set of coherent frequency bins).

Within the MFP approach there are trade-offs between various objective functions that are used to compare the measured and modeled data. The Bartlett processor is 
perhaps the most popular objective function because it is robust to inaccuracies in the simulation model inputs, but it is less precise (wider ambiguity function), resulting in decreased range resolution and therefore slightly less detailed images. Other objective functions, such as the Minimum Variance (MV), are capable of providing very precise results (narrow ambiguity) when the simulation inputs are well-known, but may also provide wildly inaccurate results when they are not.

In underwater acoustics applications, the sources are typically narrow-band and broadband sources are usually incoherent. Therefore, conventional MFP typically exploits only the spatial coherence of the field as measured by an array of sensors after performing a Discrete Fourier Transform (DFT) on the data measured by each sensor [35], [36], [44]. However, when the source is coherent across a broad band of frequencies, then the temporal coherence may also be exploited [36]. Thus, it is possible that the temporal coherence of the THz TDS spectrum could be exploited in a MFP algorithm to extract thickness information from thin film materials using only a single $\mathrm{THz}$ sensor.

MFP techniques were developed to work in complex remote sensing applications that involve in situ sensor arrays moving in noisy and dynamically changing environments, and can be characterized by several processing components [34], [35], [44]. Rather than attempting to match the modeled fields directly to the measured fields, the covariance of the measured field is often used. The covariance accounts for relative differences between the discretely sampled field measurements, and is therefore less sensitive to mis-matches due to sensor motion and/or changes in the data collection environment [35], [45].

The most common objective functions in the MFP literature include the Bartett and 
minimum variance (MV) processors [34], [35], [43]. Often a uniformly spaced grid search is used to identify a global maximum in the objective function data, and results of the search are organized to plot a 2D ambiguity surface which can be used to examine the precision and accuracy of the results. These processing techniques will be discussed in further detail in Chapter 3 .

In this dissertation, the problem of estimating thin film thickness with $\mathrm{THz}$ waves is framed in the context of MFP. Thus, it is expected that the advanced signal processing techniques that have been developed over recent decades could be valuable for thickness estimation and material property characterization with THz TDS. It does not appear that MFP techniques have been applied to THz NDE until now. Therefore, the preliminary results presented in Chapter 4 may represent the first time that MFP has been used for THz NDE.

One of the primary aims of this work is to apply the MFP techniques that have been developed in the underwater ocean acoustics community to $\mathrm{THz}$ tomography applications. Here, the objective is to use data recorded with a $\mathrm{THz}$ sensor to estimate the thickness of subsurface layers of a dielectric material, which is similar to the ocean acoustic applications of matched field tomography to estimate sediment layers.

In underwater acoustics applications, the sources are typically narrow-band and broadband sources are usually temporally incoherent. Therefore, conventional MFP typically exploits only the spatial coherence of the field as measured by an array of sensors after performing a Discrete Fourier Transform (DFT) on the data measured by each sensor [35], [36], [44]. However, when the source is temporally coherent across a broad band of frequencies, then the temporal coherence may also be exploited. Work by Tolstoy [36], Michalopoulou [46], [47], Siderius [48] and others has shown that both 
the spatial and temporal coherence of the field can be accounted for by concatenating the discrete frequency spectra from each of the sensors in an array. A summary of MFP techniques that exploit both spatial and temporal coherence has been given by Dosso [49].

Chapter 3 outlines formulations for applying the mathematical notation and methods typically found in the MFP literature to the electric field measurements that are often recorded by THz sensors. THz-TDS systems generally consist of only one sensor due to the complications involved in coherently combining data from multiple receivers in real time. Therefore, in this dissertation, MFP is used to exploit the temporal coherence of the $\mathrm{THz}$ spectrum from a single $\mathrm{THz}$ sensor to extract thickness information from thin film materials. However, the techniques presented here could also be extended to multiple sensor arrays (such as synthetic aperture arrays) using mathematical approaches in the MFP literature [49]. THz synthetic aperture imaging can be performed with a monostatic THz emitter/sensor pair that is raster scanned above the sample under test [50]-[53].

Chapter 4 presents several MFP results performed with $\mathrm{THz}$ measurement data recorded by the author in the NEAR-Lab at PSU. The temporal coherence of the $\mathrm{THz}$ waveform is expressed in terms of the covariance of the THz spectrum. The covariance matrix of the measured field is then compared with a family of modeled fields using two of the most popular processors (objective functions) in the MFP literature: the Bartlett processor and the MV processor. The precision of the estimates is evaluated with images of the ambiguity surfaces. In the axial dimension, superresolution is demonstrated by accurately estimating the thickness of a thin film of air embedded within a polymer (polycarbonate) background. 
A discussion of the significance of the results with suggestions for possible future extensions is provided in Chapter 5. It is expected that this research will provide an important link between THz NDE applications and some of the advanced physics-based signal processing methods that have already been developed in the MFP literature over the past several decades. 


\section{Chapter 3}

\section{Theoretical Background}

This chapter provides theoretical background information about topics that will be presented and discussed in the remainder of the dissertation.

\subsection{THz Time Domain Spectroscopy}

This section provides a brief summary of some of the THz TDS techniques needed to extract the index of refraction and absorption coefficient from sample materials. This is a critical step to provide the spectroscopic information needed for NDE of layer media. For NDE of thick layered media, the index of refraction is used to estimate the

phase speed and thus travel time between layer boundaries. Similarly, the index of refraction are necessary inputs for the transfer matrix model that is used to generate replica spectra for the MFP algorithms discussed in section 3.3.

Although the spectroscopic methods discussed in this section are already available in the $\mathrm{THz}$ literature, some aspects of the laboratory and signal processing techniques are still in development. Therefore, extensive effort was necessary to research the existing methods, develop experience preparing samples and designing experimental methods 
for making THz TDS measurements in the laboratory. This was especially true for $\mathrm{THz}$ spectroscopy for coating materials (e.g. paint films) which must be applied evenly with a known thickness to a substrate which also must have an extremely uniform thickness.

The complex index of refraction directly affects the wave velocity and attenuation of an electromagnetic wave traveling through a material and is denoted by

$$
\tilde{n}=n+j \kappa .
$$

The real part of $\tilde{n}$ is also called the index of refraction (denoted simply as $n$ ), and accounts for the time delay as the wave travels through the medium. For most polymer materials, the (real) index of refraction shows very little frequency dependence. However, for many polar materials, it does show some gradual frequency dependence, resulting in dispersion. The imaginary part of the index of refraction is called the extinction coefficient, $\kappa$. For many polar materials at $\mathrm{THz}$ frequencies the spectroscopic signature of the extinction coefficient is much more dramatic than that of the index of refraction (real part). The extinction coefficient indicates the amount of attenuation provided by the medium, and is therefore directly related to the power attenuation for a wave passing through the medium. The attenuation coefficient, $\alpha$, is given by

$$
\alpha=\frac{4 \pi \kappa}{\lambda}
$$

where $\lambda$ is the wavelength. 
The objective of THz TDS is to get the frequency-dependent complex index of refraction for a given sample that is representative of a material of interest. Therefore, $\mathrm{THz}$ TDS processing typically performed in the frequency domain. The pulsed $\mathrm{THz}$ system records the received THz waveform data as $r[t]$, where $t$ is a discretely sampled time axis. Then a discrete Fourier transform is performed to compute the received spectrum, $R[f]$, where $f$ is the discretely sampled frequency spectrum. The positive frequencies for which there is high SNR are then used to in subsequent processing. Usually, many pulsed $\mathrm{THz}$ waveforms are averaged improve the SNR and thus the useful bandwidth for frequency-domain processing.

A physical model is used to relate the measured spectrum, $R[f]$, to the transfer function of the sample. The physical model expresses the sample's transfer function in terms of the the $\mathrm{THz}$ source spectrum and the a number of other transfer functions for components within the $\mathrm{THz}$ systems including lenses, mirrors, etc. Analytic expressions for the $\mathrm{THz}$ source and other components are typically not available. Therefore, a separate reference measurement is performed without the sample present. Normalizing the sample measurement by the reference measurement removes these unknowns and isolates the material parameters in a process called deconvolution. Both the sample and reference measurements are typically performed in vacuum (or sometimes in nitrogen) to remove the spectroscopic absorption features of water vapor, etc.

Finally, a physical model is derived to express the transfer function in terms of the complex index of refraction of the sample material under test. The sample under test is usually chosen to be homogeneous with smooth surfaces and the sample is oriented between the source and receiver so that one can assume plane waves enter and leave the sample at normal incidence. Then the transfer function for the sample can be 
expressed in terms of the Fresnel reflection and transmission coefficients.

For an electromagnetic wave traveling from medium a, to medium $b$, at normal incidence the ratio of the transmitted to incident electric field is given by the Fresnel transmission coefficient,

$$
T_{a b}=\frac{2 \tilde{n}_{a}}{\tilde{n}_{a}+\tilde{n}_{b}},
$$

where the complex indices of refraction for medium a and medium $\mathrm{b}$, are given by $\tilde{n}_{a}$ and $\tilde{n}_{b}$, respectively. The Fresnel reflection coefficient for a plane wave reflecting of off medium $b$ and back into medium $a$ is given by

$$
\Gamma_{a b}=\frac{\tilde{n}_{a}-\tilde{n}_{b}}{\tilde{n}_{a}+\tilde{n}_{b}} .
$$

The time delay and attenuation accumulated for a plane wave propagating inside medium $b$ is given by

$$
P_{b}=\exp \left(-j \frac{\tilde{n}_{b} \omega d}{c}\right)
$$

where $c$ is the speed of light in vacuum and $\omega=2 \pi f$ where $f$ is frequency in $\mathrm{Hz}$. The distance traveled in the medium is given by $d$, and is often assumed to be known from a separate measurement such as a caliper.

This remainder of this section will consider methods to extract material properties 
from a single layer sample and from a film material (such as paint) on a substrate material. Additional details about $\mathrm{THz}$ spectroscopy techniques can be found in the literature [3], [54]-[58].

\subsubsection{THz Spectroscopy for a Thick Layer}

For many materials of interest it is possible to create a relatively rigid sample of uniform thickness with smooth planar layers. Then THz TDS measurements can be performed in transmission mode with the sample between the $\mathrm{THz}$ emitter and receiver as illustrated in the top panel of Figure 3.1.1. Here the index of refraction for the surrounding dry air or vacuum and the sample material are denoted $n_{0}$, and $n_{1}$, respectively, and the thickness of the sample is $d$. The bottom panel of Figure 3.1.1 shows the configuration for the reference measurement, where the sample has been replaced by an invisible box (dashed lines) of thickness $d$ that contains dry air, $n_{0}$. 
Sample

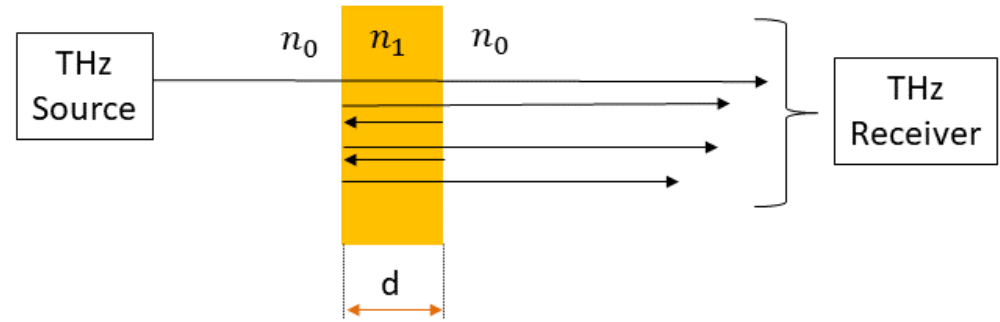

Reference

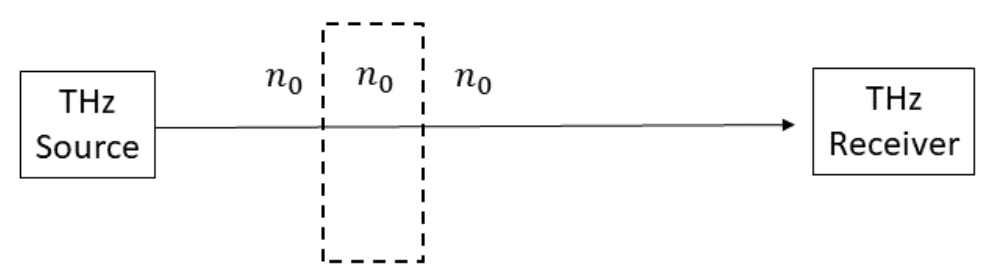

Figure 3.1.1: Sample configuration for a single layer sample in transmission mode. Top panel: A sample material with unknown $\tilde{n}_{1}$ is surrounded by an outer medium (e.g. dry air or vacuum) with $\tilde{n}_{0}$. Bottom panel: The reference measurement is made without the sample present in order to remove unknown parameters from the model.

The $\mathrm{THz}$ emitter, receiver and sample under test are carefully positioned so that plane wave propagation at normal incidence at the surfaces can be assumed. For the scenario shown in Figure 3.1.1, the physical model for the deconvolved spectrum is

$$
H=\frac{R_{\text {sample }}}{R_{\text {ref }}}=\frac{D T_{01} P_{1} T_{10} M_{1}}{D P_{0}} .
$$


The spectrum of the unknown components, $D$, can thus be removed from the model leaving the simplified expression,

$$
H=\frac{T_{01} P_{1} T_{10} M_{1}}{P_{0}}
$$

The term denoted $M_{1}$, in Eq. 3.1.6 and 3.1.7 accounts for multipath within the sample layer and is given by

$$
M=\sum_{q=0}^{Q}\left[\Gamma_{01} \Gamma_{10} P_{1}^{2}\right]^{q}
$$

It is important that the maximum number of terms, $Q$, in the summation are equivalent to the number of multipath observed in the sample waveform used for the $\mathrm{THz}$ TDS processing. If an infinite number of terms can be assumed, then $M$ may be expressed in terms of a geometric series expansion,

$$
M=\frac{1}{\left(1-\Gamma_{01} \Gamma_{10} P_{1}^{2}\right)} .
$$

Since Equation 3.1.7 is a nonlinear function of the complex index of refraction, $\tilde{n}_{1}$, it cannot be solved analytically. A numerical method for determining the index of refraction is available in the literature [55], [57], [58]. 


\subsection{2 $\mathrm{THz}$ Spectroscopy for a Film Layer on a Substrate}

For some materials of interest (e.g. paint or other coating materials) it is not possible to use the THz TDS configuration discussed in Section 3.1.1 due to the difficulty in creating a rigid sample with uniform thickness as shown in the top panel of Figure 3.1.1. The method discussed here is similar to that found in the literature [56], where the material of interest was carbon nanotube fibers. To the author's knowledge the application of this technique to extract the index of refraction from THz TDS data for a paint film on a substrate has not been published until now.

For coating materials such as paint, the sample material is applied to a substrate, thus creating a two-layer sample as shown in Figure 3.1.2. The reference measurement shown in the bottom panel of Figure 3.1.2 is performed without the sample present (i.e. substrate only) in order to remove unknown parameters from the transfer function model. 
Sample

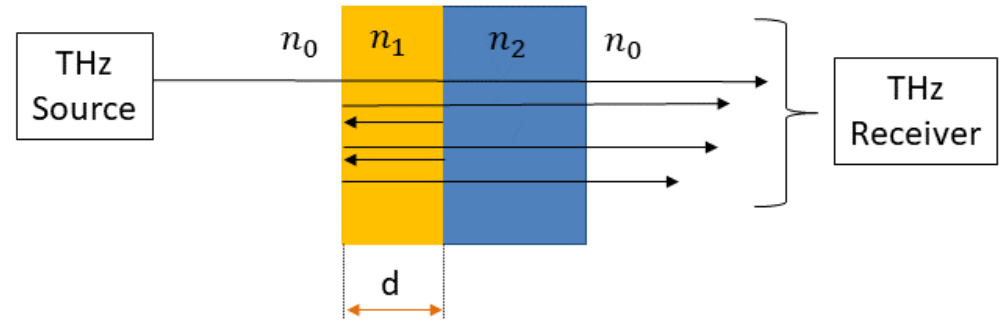

Reference

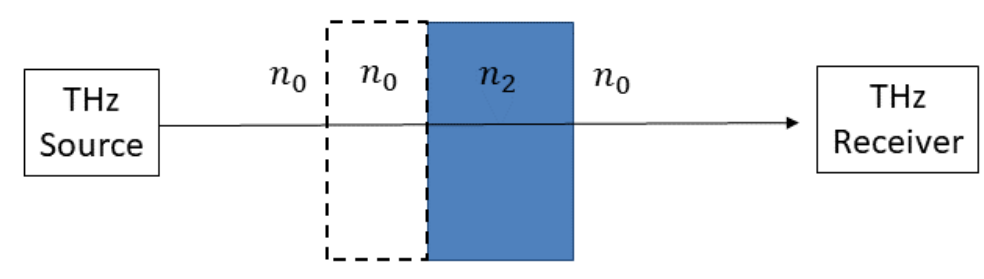

Figure 3.1.2: Sample configuration for a sample material of interest (e.g. paint) applied to a substrate (e.g. silicon wafer) that has known properties. Top panel: A sample material with unknown $\tilde{n}_{1}$ is applied to a substrate with known $\tilde{n}_{2}$. The bi-layer sample is surrounded by an outer medium (e.g. dry air or vacuum) with $\tilde{n}_{0}$. Bottom panel: The reference measurement is made without the sample present (i.e. substrate only) in order to remove unknown parameters from the model.

The transfer function for the unknown coating material in the top panel of Figure 3.1.2 is given by

$$
H=\frac{T_{01} P_{1} T_{12}}{T_{02} P_{0}\left(1-\Gamma_{10} \Gamma_{12} P_{1}\right)} .
$$

It is important to note that the expression in Equation 3.1.10 assumes infinite mul- 
tipath reflections in the coating layer, but no multipath reflections in the substrate layer. Thus, the substrate layer must be either (a) thick enough to attenuate any multipath propagation within it or (b) any multipath reflections that are due to the substrate layer must be truncated from the measured $\mathrm{THz}$ waveform for both the sample and reference measurements.

The index of refraction for the substrate, $\tilde{n}_{2}$, will be needed to compute $T_{02}$ and $T_{12}$ in Equation 3.1.10. Therefore, a separate THz TDS should be performed with the substrate alone as discussed in Section 3.1.1.

It is also important to note that $\Gamma_{a b}=-\Gamma_{b a}$. Therefore, the sign of the second term in the denominator of Equation 3.1.10 is sometimes changed in the literature [56] and Equation 3.1.10 can be expressed as

$$
H=\frac{T_{01} P_{1} T_{12}}{T_{02} P_{0}\left(1+\Gamma_{01} \Gamma_{12} P_{1}\right)} .
$$

Since Equation 3.1.10 is a nonlinear function of the complex index of refraction, $\tilde{n}_{1}$, it cannot be solved analytically. A numerical method for determining the index of refraction is available in the literature [55], [57], [58].

\subsection{THz NDE of Thick Layered Media}

In a majority of $\mathrm{THz}$ NDE applications, the refractive indices of the materials involved are known, as is the order in which the layers appear. Thus, the thickness of layers can be identified from the time delay between $\mathrm{THz}$ pulses that are reflected 
from the layer boundaries. As discussed in Section 3.2.1, some researchers have applied deconvolution in an attempt to convert the single-cycle $\mathrm{THz}$ pulse waveform to a single pulse (an approximate impulse) [8], [25], [40]-[42]. In [37], the author applied the matched filter for the detection of media boundaries in THz NDE applications. This section provides a brief derivation of the matched filtering approach. A comparison of results from Wiener deconvolution (see Section 3.2.1) and matched filtering is provided in Section 3.2 and indicates that matched filtering is more robust in measurement conditions with low SNR [37].

THz NDE research of thick layered media in the literature have often been dependent on a visual evaluation of a train of echo pulses in an A-scan to locate layer boundaries or defects [8], [12]-[14], [16], [39]. In a majority of THz NDE applications, the refractive indices of the materials involved are known, as is the order in which the layers appear. Thus, the thickness of layers can be identified from the time delay between $\mathrm{THz}$ pulses that are reflected from the layer boundaries.

Automation of the evaluation process is challenging because the $\mathrm{THz}$ pulse typically contains at least one zero-crossing between the negative and positive portions of the $\mathrm{THz}$ pulse. In addition, the reflected pulses may be inverted depending on the difference between the refractive indices (i.e. impedance mismatches) at the layer boundaries. Finally, transmission through lossy media often results in relatively low signal-to-noise ratios (SNR) making interpretation of the results a subjective process that is prone to confirmation bias. 


\subsubsection{Conventional Method: Deconvolution Processing}

This section provides a brief summary of the conventional method that often appears in the literature for THz NDE of thick layered media. Deconvolution processing (similar to THz spectroscopic analysis discussed in Section 3.1) is used to convert the single-cycle $\mathrm{THz}$ pulse waveform to a single pulse (an approximate impulse) [8], [25], [40]-[42].

For $\mathrm{THz}$ NDE of thick layered media, the transfer function, $H(f)$, of a sample is defined in the frequency domain as [3]

$$
H(f)=\frac{S(f)}{R(f)}=\left[\frac{\rho_{s}(f)}{\rho_{r}(f)}\right] e^{j\left[\phi_{s}(f)-\phi_{r}(f)\right]},
$$

where $f$ is frequency, and $S(f)$ and $R(f)$ are the Fourier transforms of the time domain sample and reference waveforms, $s(t)$ and $r(t)$, respectively. Each of the spectra in (3.2.1) are complex quantities with magnitude $\rho(f)$ and phase $\phi(f)$. The reference $\mathrm{THz}$ measurement is taken without the sample in place, to provide a transfer function of the $\mathrm{THz}$ instrumentation and the propagation channel. Thus, dividing the sample spectrum by the reference spectrum yields an estimate of the transfer function of the sample alone. In a reflection measurement the reference waveform is measured by replacing the sample with a conductive mirror.

The inverse Fourier transform of the transfer function provides an estimate of the sample's impulse response. $\mathrm{THz}$ echo pulses appear within the impulse response wherever there is an impedance mismatch within the media; potentially indicating the presence of a defect in a background material or a new layer in a sample composed of layered media. 
The layer thickness, $d$ can then be calculated from the two-way travel time, $\tau$, between impulses as

$$
d=2 v_{g} \tau,
$$

where the group velocity of the sample medium, $v_{g}=c / n_{g}$, depends on the speed of light in vacuum, $c$, and the group index of refraction,

$$
n_{g}(f)=n(f)+f \frac{d}{d f} n(f) .
$$

where $n(f)$ is the real part of the complex index of refraction.

Industrial applications of $\mathrm{THz}$ NDE methods will likely require many sample measurements to be recorded very quickly. This can be accomplished by reducing the integration time (e.g. dwell time at each image pixel) at the expense of reduced SNR in the sample measurements. However, it is well-known that dividing the sample spectrum by the reference spectrum, as in (3.2.1), results in amplification of noise wherever the SNR of the sample measurement is low [8]. This results in high intensity peaks in the impulse response, $h(t)=F^{-1}[H(f)]$. Therefore, inverse filters are sometimes used to suppress noise, especially at high frequencies. The simplest approach to inverse filtering is to use Wiener deconvolution, where the transfer function in $(3.2 .1)$ is written as [8]

$$
H(f)=S(f) W(f),
$$

and $W(f)$ is the Wiener deconvolution filter given by

$$
W(f)=\frac{R^{*}(f)}{R(f) R^{*}(f)-\left(\frac{1}{\operatorname{SNR}(f)}\right)} .
$$

In (3.2.5) the asterisk represents the complex conjugate and SNR refers to the signal 
to noise ratio of the sample spectrum.

Some THz researchers have suggested that improvements are possible with more complex filters [8], [25], [40]-[42]. These filters use more parameters that depend on the spectrum of the THz reference waveform (which is unique to each THz TDS system), and can be relatively complex to implement. While such efforts have been helpful in advancing THz NDE, the techniques can be difficult to apply in general. Designing and optimizing a filter that attenuates the anomalous peaks while preserving the main peak can be time-consuming and may not be entirely successful at removing all of the ringing artifacts [8].

In Section 3.2.2, the author proposes using a matched filter to estimate the impulse response. A comparison of results from Wiener deconvolution and matched filtering (see Section 3.2) indicates that matched filtering is more robust in measurement conditions with low SNR [37].

It is important to note that in thin layered media, the $\mathrm{THz}$ pulses that are reflected from the front and back surfaces overlap with one another, and the methods presented in this section cannot be used. A proposed approach for $\mathrm{THz}$ NDE of thin layered media is presented in Section 3.3.

\subsubsection{Proposed Approach: Matched Filter}

The objective of boundary or defect detection in THz NDE can be framed in terms of statistical signal processing, in which the goal is to use the source signal and the signal measured from a sample to estimate a parameter or vector of parameters. The

parameter(s) that maximize the output of the matched filter are then used to identify 
whether or not an echo pulse is present in the received signal.

The MLE makes a comparison of two signals: the source signal and a measured signal, which is modified from of the source signal as it passes through the propagation channel. At the receiver, the measured signal must compete with noise. In a THz TDS system the propagation channel is considered to be only the portion of the propagation path within the sample media, i.e. excluding the photo-conductive antenna and all lenses, etc.

For THz TDS, the source (or "template") signal may be estimated by the reference waveform, $r(t)$. The $\mathrm{THz}$ reference signal is itself corrupted by noise in the $\mathrm{THz}$ receiver and is therefore only an estimate of the pure source signal. However, averaging many reference waveforms can significantly reduce the noise. Unlike the sample measurements, the reference only needs to be measured once. Therefore, a longer integration time is acceptable for this measurement.

The THz TDS signal measured with the sample in place, $s(t)$, is a modified form of the reference waveform that has been delayed and attenuated by propagation through the sample media. Typically, the sample signal is collected with a lower averaging time (for example to increase the speed of data collection during raster scan imaging) and therefore the noise, $n(t)$, added at the THz TDS detector cannot be neglected.

Thus, after a THz source signal, $r(t)$, passes through a sample material (in either reflection or transmission configuration) the signal received at the THz TDS detector, $s(t)$, can be expressed as

$$
s(t)=r(t, \boldsymbol{\theta})+n(t),
$$

where $n(t)$ represents the noise at the $\mathrm{THz}$ detector and $r(t, \boldsymbol{\theta})$ represents the $\mathrm{THz}$ 
reference waveform, $r(t)$, modified with the parameter(s) contained in the vector, $\boldsymbol{\theta}$. The analytic form of (3.2.6) is given by

$$
s_{a}(t)=\alpha_{0} r_{a}(t, \boldsymbol{\theta})+n_{a}(t)
$$

where $s_{a}(t), r_{a}(t, \boldsymbol{\theta})$ and $n_{a}(t)$ are the analytic forms of $s(t), r(t, \boldsymbol{\theta})$ and $n(t)$, respectively, and $\alpha_{0}$ is a complex coefficient to account for attenuation and delay in the propagation path.

The MLE produces a measure of the likelihood, $Q$, that the specific parameter, $\boldsymbol{\theta}_{0}$, produced the signal $s_{a}(t)$ from the source signal, $r_{a}(t)$, in the presence of noise that is described by $n_{a}(t)[31]$ :

$$
Q\left(\boldsymbol{\theta}, \boldsymbol{\theta}_{0}\right)=\frac{\left|\int s_{a}(t, \boldsymbol{\theta}) r_{a}^{*}\left(t, \boldsymbol{\theta}_{0}\right) d t\right|^{2}}{\int\left|r_{a}^{*}(t, \boldsymbol{\theta})\right|^{2} d t}
$$

By examining (3.2.8) a means of assessing the performance of the MLE for a given measurement system can be obtained [31]. Assuming the denominator of (3.2.8) is unity, then (3.2.8) can be expressed as

$$
Q\left(\boldsymbol{\theta}, \boldsymbol{\theta}_{0}\right)=A_{0}\left|X\left(\boldsymbol{\theta}, \boldsymbol{\theta}_{0}\right)+Y(\boldsymbol{\theta})\right|^{2}
$$

where $A_{0}=\left|\alpha_{0}\right|^{2}$ represents the energy of the received signal and

$$
X\left(\boldsymbol{\theta}, \boldsymbol{\theta}_{0}\right)=\int r_{a}(t, \boldsymbol{\theta}) r_{a}^{*}\left(t, \boldsymbol{\theta}_{0}\right) d t
$$


and

$$
Y(\boldsymbol{\theta})=\frac{1}{\alpha_{0}} \int n_{a}(t) r_{a}^{*}\left(t, \boldsymbol{\theta}_{0}\right) d t
$$

If $n_{a}(t)$ is assumed to be a zero-mean Gaussian random variable, then the power of $Y(\boldsymbol{\theta})$ is $N_{0} / A_{0}$ where $N_{0}$ is the noise spectral density. In this case, it can be shown [31] that the expected value of the estimator in (3.2.9) is

$$
E\left[Q\left(\boldsymbol{\theta}, \boldsymbol{\theta}_{0}\right)\right]=A_{0}\left|X\left(\boldsymbol{\theta}, \boldsymbol{\theta}_{0}\right)\right|^{2}+N_{0}
$$

The performance of the estimator can be evaluated by considering the expected value of $Q\left(\boldsymbol{\theta}, \boldsymbol{\theta}_{0}\right)$ in the ideal case where $N_{0}=0$. In this case, the performance of the estimator is characterized by the ambiguity function, $\left|X\left(\boldsymbol{\theta}, \boldsymbol{\theta}_{0}\right)\right|^{2}[31],[32]$. The ambiguity function is generally composed of a peak (near the center of the parameter domain) with sidelobes that indicate the potential for false detections. The width of the main peak of the ambiguity function defines the minimum resolution of the parameter to be estimated.

For many $\mathrm{THz}$ NDE applications, the sample and measurement system are both stationary and the objective is to detect only the boundary between the layers of stratified media. In such simple cases, the parameter vector reduces to a single element, time delay, $\boldsymbol{\theta}=[\tau]$. Under this assumption, the integral inside the absolute value brackets in the numerator of (3.2.8) is simply a cross-correlation of the analytic $\mathrm{THz}$ TDS sample and reference waveforms, and the denominator is a normalization constant. Thus, the MLE reduces to a matched filter that compares the analytic 
(complex) THz sample signal with the analytic $\mathrm{THz}$ reference signal,

$$
Q\left(\tau, \tau_{0}\right)=\left|\int s_{a}(t, \tau) r_{a}^{*}\left(t, \tau_{0}\right) d t\right|^{2}=\left|m_{a}(t)\right|^{2},
$$

where $m_{a}(t)$ is the analytic matched filter output. Thus, in this special case, the ambiguity function, based on (3.2.10), becomes the squared magnitude of the autocorrelation function for the analytic $\mathrm{THz}$ reference pulse.

It is important to note that the frequency domain matched filter output, $M(f)$, from the sample and reference spectra is denoted

$$
M(f)=\mathcal{F}\left\{m_{a}(t)\right\}=\rho_{s}(f) \rho_{r}(f) e^{j\left[\phi_{s}(f)-\phi_{r}(f)\right]},
$$

where $\mathcal{F}\{\}$ indicates Fourier transform. $M(f)$ contains exactly the same phase term as in (3.2.1), which is of primary interest for most THz NDE applications. However, unlike in (3.2.1), the magnitude of $M(f)$ is not complicated by the noise amplification problem that results from taking the ratio of the magnitudes of the sample and reference spectra. Therefore, the MLE, $Q\left(\boldsymbol{\theta}, \boldsymbol{\theta}_{0}\right)$, experiences less noise corruption than the transfer function, $h(t)$, and the processing is simplified by eliminating the need for a Wiener filter or other inverse filtering techniques to suppress noise.

\subsection{THz NDE of Thin Films}

As discussed in the previous section, THz TDS systems can be used for NDE of thick layered media, but challenges arise when then the layer thickness is less than the delay spread between multipath arrivals is less than the pulse width. In thin film samples, 
$\mathrm{THz}$ pulses that are reflected from the front and back surfaces of the layer overlap on one another and the time delay between them (and thus propagation distance, thickness) cannot be determined. This problem can become even more complicated when there are multiple thin films stacked on top of one another. Examples include measuring the thickness of paint films or other thin layers in laminated media.

\subsubsection{Matched Field Processing (MFP) Approach}

The author's approach to extract thickness information from THz TDS measurement from a thin film sample is to apply the MFP algorithm, which has been primarily used for remote sensing applications in underwater ocean acoustics. In the remainder of this section each of the four components of MFP are discussed in the context of THz NDE of thin-film layered media.

\subsubsection{Measurement Data from Pulsed $\mathrm{THz}$ Sensors}

This disseration is focused on the development of new physics-based signal processing approaches and techniques, and therefore no new $\mathrm{THz}$ measurement hardware is developed for this work. Rather, experiments were performed with the Picometrix T-Ray 4000 THz system in the NEAR-Lab at PSU. Similar commercial systems are currently being used by research scientists around the world.

Pulsed THz systems generally consist of only one (1) sensor because of the complications involved in coherently combining data from multiple receivers in real time. However, one of the primary features of these systems is their ability to maintain temporal coherence over a wide spectral bandwidth. Therefore, the MFP algorithm 
introduced in section 2.2 can exploit this temporal coherence, in either time domain or (temporal) frequency domain, to match measured $\mathrm{THz}$ data with replica data that is synthesized with propagation models. The next section will discuss propagation modeling for $\mathrm{THz}$ waves in layered media, and some common objective functions that can be used to compare modeled replicas with measurement data are presented in Chapter 4 .

\subsubsection{Mathematical Model for Terahertz Measurement Data}

For a THz-TDS system, the received time-domain waveform can be expressed as a function of time, $t$, and the parameters of interest for the sample under test. In general, there can be many parameters, $p_{1}, p_{2}, \ldots$, and therefore it is convenient to denote them as a single vector, $\boldsymbol{a}=\left[p_{1}, p_{2}, p_{3}, \ldots\right]^{T}$, where the superscript $T$ represents the matrix transpose operation. The true parameters of the sample are denoted $\boldsymbol{a}_{T}$, and MFP is used to compare measured and modeled field data using an objective function to arrive at a best estimate the parameter vector, denoted $\hat{\boldsymbol{a}}$.

Therefore, the received electric field, $r\left(t, \boldsymbol{a}_{T}\right)$, can be expressed as the convolution

of the source signal, $s(t)$, and the impulse response, $h\left(t, \boldsymbol{a}_{T}\right)$, and the addition of measurement noise, $n(t)$. Thus, the received time domain waveform is modeled as

$$
r\left(t, \boldsymbol{a}_{T}\right)=s(t) * h\left(t, \boldsymbol{a}_{T}\right)+n(t)
$$

where $*$ represents convolution. In Eq. 3.3.1, the source and noise signals are independent of the sample under test and therefore independent of the parameter vector, $\boldsymbol{a}_{T}$. 
After a Fourier transform Eq. 3.3.1 can be expressed in the frequency domain as

$$
R\left(f, \boldsymbol{a}_{T}\right)=S(f) H\left(f, \boldsymbol{a}_{T}\right)+N(f),
$$

where $f$ is the frequency in $\mathrm{Hz}, R\left(f, \boldsymbol{a}_{T}\right)$ is the received spectrum, and $S(f)$ and $N(f)$ are the source and noise spectra, respectively.

The transfer function, $H\left(f, \boldsymbol{a}_{T}\right)$, can be expressed as the convolution of several other transfer functions that each describe the performance of the various components of the $\mathrm{THz}$ system and the propagation channel of the THz beam as it travels through the sample to the receiver. Thus, the received spectrum is

$$
R\left(f, \boldsymbol{a}_{T}\right)=S(f) H_{T x}(f) H_{s}\left(f, \boldsymbol{a}_{T}\right) H_{R x}(f)+N(f),
$$

where $H_{T x}(f)$ and $H_{R x}(f)$ are transfer functions that account for the components components of the $\mathrm{THz}$ transmitter and receiver, respectively. Depending on the specific hardware configuration these components may include broadband antennas that radiate and receive the electric fields and lenses that focus the $\mathrm{THz}$ beams. In Eq. 3.3.3, the transfer function, $H_{s}\left(f, \boldsymbol{a}_{T}\right)$ accounts for propagation within the sample under test.

In general, the propagation in the background of the channel can be relatively complicated because it must account for dispersion and attenuation in the air. Similarly, it is difficult to quantify the $\mathrm{THz}$ source spectrum and the other transfer functions of the pulsed THz system in Eq. 3.3.3. Therefore, it is common practice to group these unknown factors together into a single term and express the received spectrum more compactly as 


$$
R\left(f, \boldsymbol{a}_{T}\right)=D(f) H_{s}\left(f, \boldsymbol{a}_{T}\right)+N(f),
$$

where $D(p, f)$ represents the convolution of the source spectrum, $S(f)$, and the transfer functions that accounts for propagation through the various components of the $\mathrm{THz}$ measurement system and the background medium, excluding the sample under test.

The unknown system variables in $D(f)$ can be accounted for with a reference waveform that is collected with a known sample having a transfer function, $H_{s}(f)$, that does not depend on the parameter vector. Many measurements of the reference waveform are collected and the received spectrum is averaged over a long integration time to reduce the effects of measurement noise. This longer integration time is acceptable in THz NDE applications because, unlike the sample measurements, this reference only needs to be recorded once for a given measurement system. However, it should be repeated periodically to account for changes in $S(f)$ that can occur on a longer time scale.

Thus, after averaging many measurements, the mean received field can be approximated as

$$
R_{r e f}\left(f, \boldsymbol{a}_{T}\right) \approx D(f) H_{s}\left(f, \boldsymbol{a}_{T}\right),
$$

For a $\mathrm{THz}$ system configured in reflection mode, the reference signal is measured with the sample replaced by a mirror positioned as close as possible to same position as the surface of the sample. Then the transfer function of the sample is simply, $H_{s}(f) \approx-1$. Therefore, $D(f) \approx-R_{\text {ref }}(f)$, and the received spectrum for a given 
layered media sample can finally be expressed as

$$
R\left(f, \boldsymbol{a}_{T}\right)=-R_{r e f}(f) H_{s}\left(f, \boldsymbol{a}_{T}\right)+N(f) .
$$

\subsubsection{Generating THz Replica Spectra}

The MFP algorithm requires a forward propagation model to generate replicas of the $\mathrm{THz}$ spectrum that can be later compared with the measured $\mathrm{THz}$ spectrum by an objective function. Various propagation models could be used within MFP depending on the geometric configuration of the source, sample and sensor(s). A propagation model for the transfer function, $H_{s}(f)$, for a parallel stack of layered media is presented in Appendix A.

The transfer function in Eq. A.2.8 can be parameterized in terms of variables within the model. For example, $H_{s}(f, \boldsymbol{a})$, where $\boldsymbol{a}=\left[d_{1}, d_{2}, \ldots d_{Q}\right]^{T}$, with the thickness of each layer, $d_{q}$, used in Eq. A.2.4 of the model. Similarly, the refractive index in Eq. A.2.3 could be be further parameterized using the Lorentz model, Drude model, and/or various effective media models that can account for random scattering within the layer(s) using an effective index of refraction.

Thus, the model presented in this section can be used to generate a set of simulated replica fields,

$$
R_{r}(f, \boldsymbol{a})=D(f) H_{s}(f, \boldsymbol{a}),
$$

where the subscript, $r$, denotes replica field and $D(f)$ is obtained from a reference measurement as discussed above. 
In practice, the measured field is discretely sampled by the $\mathrm{THz}$ measurement system, with the total of $L$ time samples in the measured waveform. Then the Fourier transforms discussed in the previous section can be realized as a discrete Fourier transform (DFT) that results in discrete frequency bins. The discrete spectrum can then be truncated to a bandwidth of $L$ frequency bins within which there is sufficient signalto-noise ratio (SNR). Thus, the discrete spectrum of the received spectrum can be denoted as a vector, $\boldsymbol{R}\left(\boldsymbol{a}_{\boldsymbol{T}}\right)$, of length, $L$. Note, the dependence on frequency, $f$, is assumed in the vector notation.

Similarly, the replica fields that are generated with Eq. 3.3 .7 can be modeled at the same discrete frequencies as the measured field (Eq. 3.3.6) for direct comparison using an objective function. Thus, the replica fields can also be expressed as vectors, $\boldsymbol{R}_{\boldsymbol{r}}(\boldsymbol{a})$, of length L.

It is important to note that the propagation model in Appendix A.1, inherently includes an infinite number of multipath reflections in each layer [59], and undersampling the field in the frequency domain can result in aliased multipath arrivals in the modeled field data. Therefore, the modeled fields should be generated at small frequency intervals, and then an inverse transform should be performed to project the modeled fields into the time domain where they can be truncated at the same duration as the measured fields. Finally, a DFT is performed on the truncated replica waveforms to create the replica spectra, $\boldsymbol{R}_{\boldsymbol{r}}(\boldsymbol{a})$. These replica spectra are then truncated to include the same bandwidth as the the measured spectrum, $R\left(\boldsymbol{a}_{T}\right)$.

Finally, a normalized weight vector, $\boldsymbol{w}(\boldsymbol{a})$, is created from the replica field,

$$
\boldsymbol{w}(\boldsymbol{a})=\frac{\boldsymbol{R}_{r}(\boldsymbol{a})}{\left|\boldsymbol{R}_{r}(\boldsymbol{a})\right|}
$$




\subsubsection{Sample Covariance Matrix}

The weight vector could be compared with the measured field vector, but it is more common in the MFP literature to use the covariance of the measured field. The covariance accounts for relative differences between the discretely sampled field measurements, and is therefore less sensitive to mis-matches due to sensor motion and/or changes in the data collection environment [35], [45]. The covariance matrix of the measured field is approximated as

$$
\hat{\boldsymbol{K}}\left(\boldsymbol{a}_{T}\right)=\frac{1}{Z} \sum_{z=1}^{Z}\left(\boldsymbol{R}_{z}\left(\boldsymbol{a}_{\boldsymbol{T}}\right) \times \boldsymbol{R}_{\boldsymbol{z}}^{\boldsymbol{H}}\left(\boldsymbol{a}_{\boldsymbol{T}}\right)\right)
$$

where $\boldsymbol{R}_{\boldsymbol{z}}\left(\boldsymbol{a}_{\boldsymbol{T}}\right)$ is the measured field for each of the $Z$ snapshots, and the superscript $H$ indicates Hermetian transpose.

The covariance is a square $[L \times L]$ matrix composed of complex numbers. Each element of the covariance matrix contains information about the relationship of one discretely sampled bin with one of the other bins. Therefore, the elements along the diagonal of the covariance matrix are the self-terms, and the covariance matrix is

complex symmetric along the diagonal. Elements of $\hat{\boldsymbol{K}}\left(\boldsymbol{a}_{T}\right)$ with large covariance values indicate a stronger relationship between the two measurement bins than an element with a covariance that is near zero.

Ideally, each of the rows (or columns) of the covariance matrix should be linearly independent, i.e. the covariance matrix should be full rank. A rule of thumb is to ensure that the total number of snapshots is greater than the length of the measured data vector, i.e. $Z>L$. This is sometimes used as a lower limit for the total number of snapshots required. 


\subsubsection{Objective Functions}

In the MFP, an objective function, sometimes called a cost function or "processor", is used to compare each of the replicas, with the measurement data, and then a search algorithm is used to find the replica field that is the best match to the measurement. In this section two of the most popular processors in the MFP literature are the Bartlett processor and the MV processor are briefly discussed.

In addition to the Bartlett processor and MV processor, a variety of other processors have also been used in the MFP literature [35], [36], [44]. The trade-offs between various other objective functions is beyond the scope of this dissertation, but can be found in the MFP literature [35], [36], [44].

\subsubsection{Bartlett Processor}

The Bartlett processor is an perhaps the most widely used processor in the MFP literature [35], [36], [44]. It can be expressed as the average of the projection of the measured data vectors on the normalized replica vectors [35],

$$
P_{B}(\boldsymbol{a})=\frac{1}{Z} \sum_{z=1}^{Z}\left|\boldsymbol{w}^{H}(\boldsymbol{a}) \boldsymbol{R}_{\boldsymbol{z}}\left(\boldsymbol{a}_{\boldsymbol{T}}\right)\right|^{2}
$$

which can be computed in with the covariance matrix as follows:

$$
P_{B}(\boldsymbol{a})=\boldsymbol{w}^{H}(\boldsymbol{a}) \hat{\boldsymbol{K}}\left(\boldsymbol{a}_{T}\right) \boldsymbol{w}(\boldsymbol{a})
$$


The trial parameter vector, $\boldsymbol{a}$, that results in a global maximum in $P_{B}(\boldsymbol{a})$ is regarded as the best estimate for the parameter(s) and is denoted, $\hat{\boldsymbol{a}}_{\boldsymbol{B}}$.

The Bartlett processor is robust to modeling inaccuracies and is relatively straightforward to implement [36] .

\subsubsection{Minimum Variance (MV) Processor}

The MV processor (also known as Capon processor) [35], [36], [44] is an adaptive processor that suppresses ambiguities. It can be expressed as

$$
P_{M V}(\hat{\boldsymbol{a}})=\frac{1}{\boldsymbol{w}^{H}(\hat{\boldsymbol{a}}) \hat{\boldsymbol{K}}^{-1}\left(\boldsymbol{a}_{T}\right) \boldsymbol{w}(\hat{\boldsymbol{a}})} .
$$

The trial parameter vector, $\boldsymbol{a}$, that results in a global maximum in $P_{M V}(\boldsymbol{a})$ is regarded as the best estimate for the parameter(s) and is denoted, $\hat{\boldsymbol{a}}_{\boldsymbol{M} \boldsymbol{V}}$.

The MV processor is capable of providing very precise results (narrow ambiguity) when the simulation inputs are well-known, but may provide inaccurate results when they are not.

It is important to note that the MV processor requires the inverse of the sample covariance matrix, $\hat{\boldsymbol{K}}^{-1}$ to be computed. This can be problematic if the sample covariance matrix is not full rank, which can happen when there is an insuffient number of snapshots included in the averaging performed in Eq. 3.3.9. If there is insufficient time available to collect additional snapshots, then a small quantity can be added to the diagonal elements of the covariance matrix, which is often referred 
to as diagonal loading. More details about diagonal loading can be found in Chapter 3 of $[36]$.

\subsubsection{Ambiguity Surfaces}

The parameter(s) that results in the optimum output from the objective function is identified as the best estimate of the measured variable. There may be multiple local peaks in the ambiguity surface and it is therefore important that replicas are generated for the entire region of feasible values of each of the free variables in the

parameter vector, $\boldsymbol{a}$, and that the search algorithm identifies the global maximum in the resulting ambiguity data.

In addition to identifying the parameter values that created the global peak in the ambiguity surface, analyzing the ambiguity in the neighborhood of the peak value give some indication of the confidence in (or precision of) the estimated parameter values. Therefore, plots of the ambiguity surfaces often appear in the MFP literature [35], [36], [44]. If there is only one free parameter in the MFP algorithm, then the ambiguity surface is simply a line plot. If there are two free parameters the ambiguity surface is generally shown as a 2D image. For MFP with more than 2 dimensions, it is possible to explore the multi-dimensional ambiguity space by selecting the peak value in all but 2 of the dimensions and then plotting the $2 \mathrm{D}$ ambiguity surface vs. the 2 remaining dimensions. 


\section{Chapter 4}

\section{Implementation, Demonstration, and Analysis}

Note:

- Section 4.1, in part, is published: S. Schecklman, G. Kniffin, and L. M. Zurk, "Terahertz non-destructive evaluation of layered media with the maximum likelihood estimator," in 2014 International Symposium on Optomechatronic Technologies, 2014, pp. 81-85.

- Section 4.2, in part, is published: S. Schecklman and L. M. Zurk, "Terahertz imaging of thin film layers with matched field processing," MDPI Sensors Journal, vol. 18, no. 10, p. 3547, Oct. 2018.

This chapter consists of three main sections describing the work performed to demonstrate the performance of the proposed approaches that were discussed in the previous chapter. Each of the examples shown here use real experimental data recorded with the T-Ray 4000 in the THz lab at PSU for NDE samples designed and constructed by the author. All signal processing algorithms are implemented with MATLAB code. 


\subsection{THz NDE of Thick Layered Media}

One of the most fundamental applications for $\mathrm{THz} \mathrm{NDE}$ is the detection of boundaries between thick layers of planar media using a reflected $\mathrm{THz}$ beam at normal incidence. In this section, samples made of Polymethyl methacrylate (PMMA), also known as acrylic, are evaluated to directly compare results of Wiener Deconvolution (discussed in Section 3.2.1) with the matched filter approach proposed in Section 3.2. Each sample is composed of smooth parallel surfaces, making them ideal for a variety of layer configurations for $\mathrm{THz}$ laboratory measurements. An initial transmission measurement with a thick sample of acrylic was performed to extract the complex index of refraction as discussed in section 3.1. The resulting complex index of refraction and absorption coefficient are shown in Figure 4.1.1. 

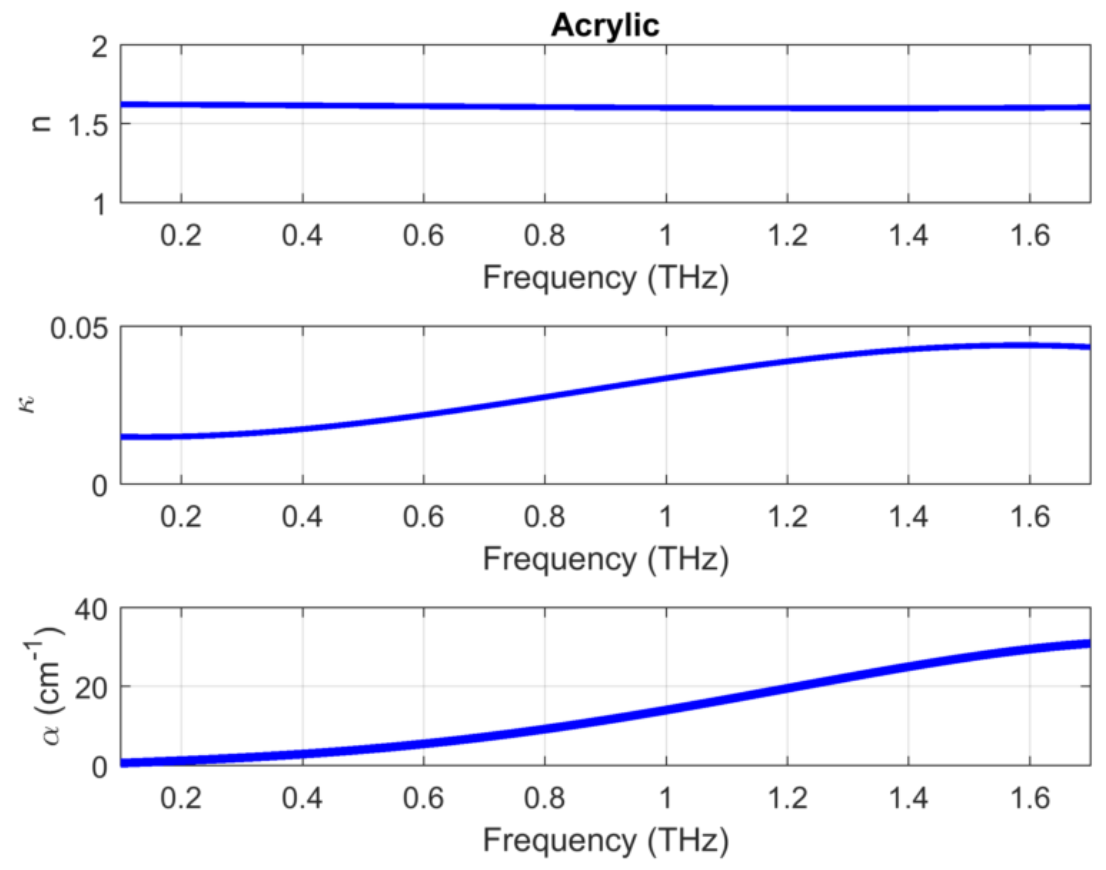

Figure 4.1.1: Complex index of refraction for Acrylic were calculated from $\mathrm{THz}$ measurement data. Top panel: real part of the refractive index, $n$. Middle Panel: Extinction coefficient, $\kappa$. Bottom Panel: Absorption coefficient $\alpha$. Results are consistent with measurements in the literature [60].

The THz reference waveform was measured once and used to process all of the sample data for both the Wiener deconvolution and matched filter methods. Figure 4.1.2 shows the reference waveform from a $\mathrm{THz}$ reflection measurement. The waveform is the result of averaging 10,000 waveforms to maximize the SNR. The resulting matched filter ambiguity function is shown in the right panel of Figure 4.1.2. Note, the ambiguity function for the $\mathrm{THz}$ pulse is a relatively narrow peak with no visible sidelobes. 

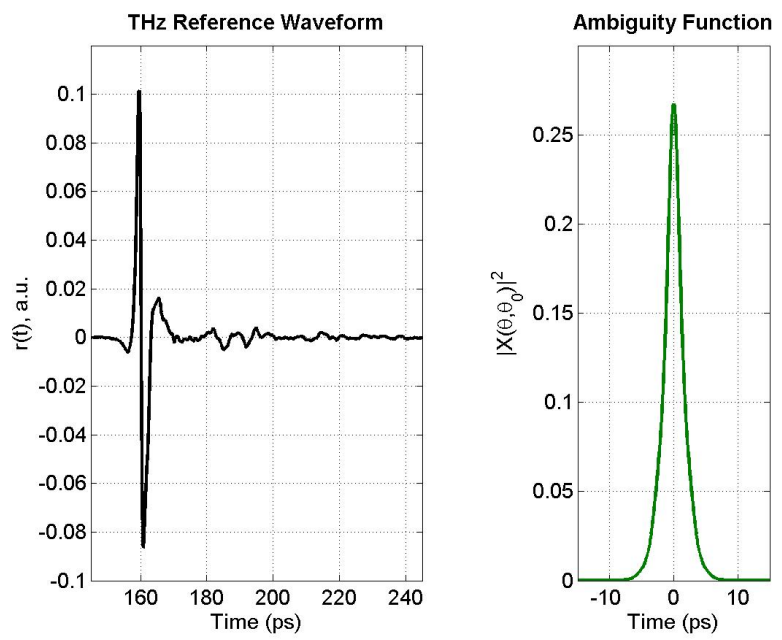

Figure 4.1.2: Left Panel. Reference waveform from $\mathrm{THz}$ reflection measurement. Right Panel. The ambiguity function (or equipment function) for the $\mathrm{THz}$ reference waveform is used to evaluate the potential performance of the maximum likelihood ratio estimator.

\subsubsection{Experiment Samples and Measurement Configuration}

The $\mathrm{THz}$ measurement system in the NEAR-Lab was used to record a $\mathrm{THz}$ waveform reflected at normal incidence from a stack of planar-layered media above a metal substrate as illustrated in Figure 4.1.3, where the numbered circles identify each of the boundaries. All of the experimental data presented here were collected using a collinear measurement head that contains transmit and receive modules joined with a duplexer into a single collocated unit for monostatic measurements. The collinear head was fit with a collimating lens and oriented to provide a $\mathrm{THz}$ beam at normal incidence on an adjustable sample stage. The stage was mounted to an optical workbench and adjusted in 3 dimensions (yaw, pitch, and roll) so that the sample media was perpendicular to the $\mathrm{THz}$ beam.

For all of the experiments the spacing of the two acrylic layers was $d_{1}=d_{3}=5.92 \mathrm{~mm}$ 
and the thickness of the lower air layer, was created by inserting shims with thickness, $d_{4}=5.62 \mathrm{~mm}$, between boundary 4 and 5 . The spacing of the air gap between the two acrylic layers (boundaries 2 and 3) was changed by inserting shims of various thickness, $d_{2}=5.62$ and $d_{2}=0.70 \mathrm{~mm}$. The thickness of the acrylic layers and each of the shims was measured with a digital Vernier caliper with $10 \mu m$ resolution and $20 \mu m$ accuracy.

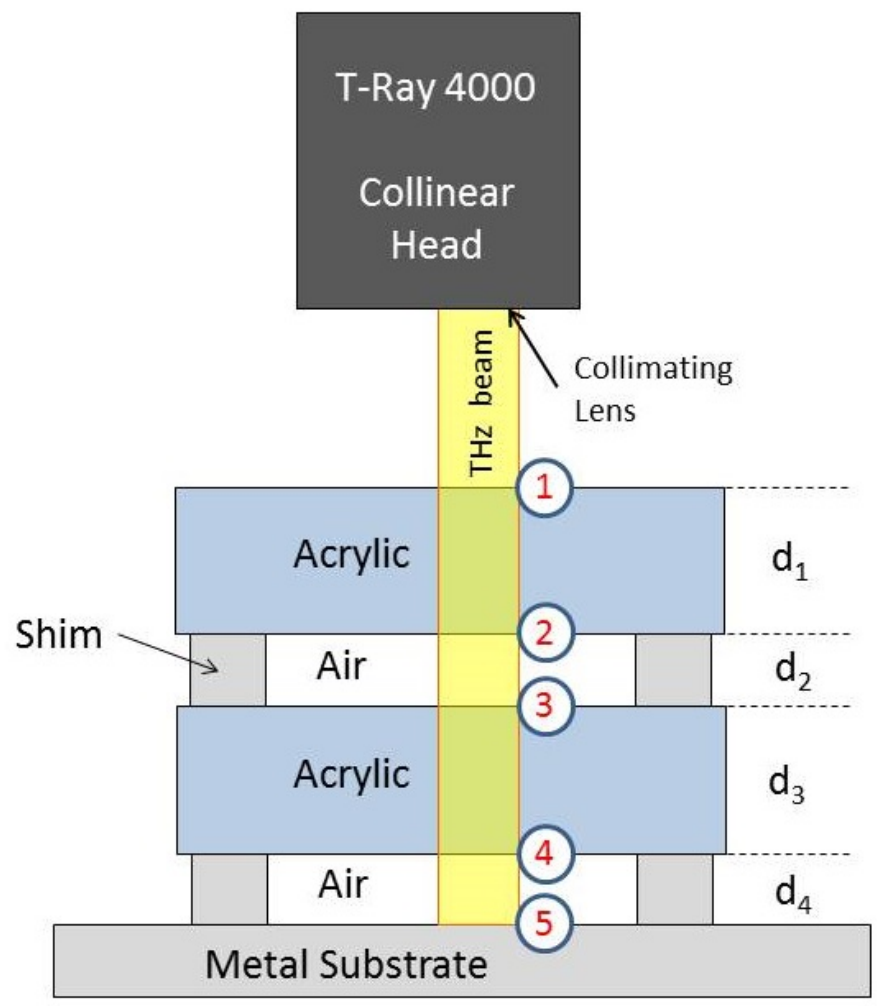

Figure 4.1.3: Illustration of the experiment configuration (not to scale). The numbered circles identify each of the boundaries. For each of the experiments, the spacing of the two acrylic layers was $d_{1}=d 3=5.89 \mathrm{~mm}$ and the thickness of the lower air layer, was $d_{4}=5.62 \mathrm{~mm}$. The spacing of the air gap between the two acrylic layers was changed by inserting shims of various thickness, $d_{2}=5.62$ and $d_{2}=0.70 \mathrm{~mm}$.

The shortest possible integration time of the T-Ray 4000 (10 ms per sample measure- 
ment) was used to collect a single sample waveform for each scenario. Note, only one set of measurements was taken for each measurement scenario so that the two signal processing methods (Weiner deconvolution and matched filtering) could be performed on the same data sets for direct comparison.

\subsubsection{THz Signal Processing Results: A-Scans}

Figure 4.1.4 shows signal processing results for $d_{2}=5.62 \mathrm{~mm}$ with the measured $\mathrm{THz}$ waveform before processing (top panel) and after processing with a conventional Wiener deconvolution filter (middle panel) and the proposed method using the matched filter (bottom panel). The layer interfaces are difficult to identify in the unprocessed waveform due to poor SNR. The time delay between the peaks is also difficult to establish due to oscillations within the envelope of each echo pulse. Arrival times are easier to identify in the waveform processed with the Wiener deconvolution method (middle panel), but peaks are still difficult to identify due to poor SNR and oscillations introduced in the baseline. The waveform resulting from the matched filter (bottom panel) show peaks occurring at delay times that are consistent with boundaries 1, 2, 3, 4 and 5, respectively (see Figure 4.1.3). Additional peaks after the fifth peak are due to multipath reflections within the layer stack.

It should be noted that the matched filter results shown in the bottom panel of Figure 4.1.4 maximize the SNR because it is based on the MLE, whereas the result of the Weiner deconvolution shows narrower peaks because the Wiener filter is based on the least square error estimator. Thus, the peaks of the matched filter output are wider than those resulting from Wiener deconvolution. 
Figure 4.1.5 shows A-scan comparisons when $d_{2}=0.70 \mathrm{~mm}$. Again, the results based on the proposed MLE approach show better SNR than the raw $\mathrm{THz}$ waveform and the conventional Wiener deconvolution approach.

THz Sample Waveforms: $d=5.62 \mathrm{~mm}$ Unprocessed Waveform
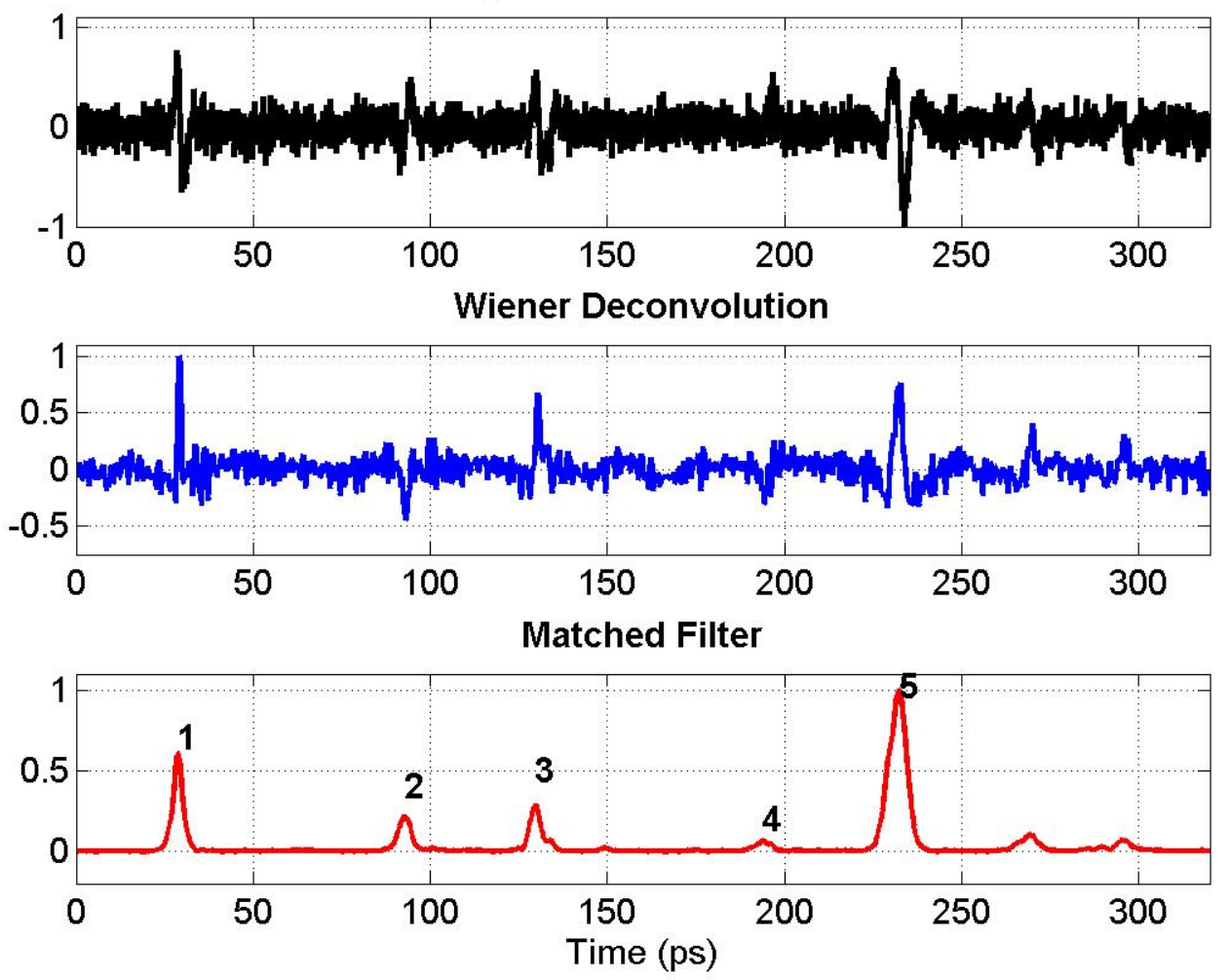

Figure 4.1.4: Comparison of results when $d_{2}=5.62 \mathrm{~mm}$. THz waveform reflected from multiple layers of planar media (top panel), the same waveform after processing with the Wiener deconvolution filter (middle panel) and after processing with the matched filter (bottom panel). The matched filter provides peaks with superior SNR. The numbers near each peak correspond to the circled boundary numbers in Figure 4.1.3. Each of the waveforms was normalized for comparison. 
THz Sample Waveforms: $d=700 \mu \mathrm{m}$ Unprocessed Waveform
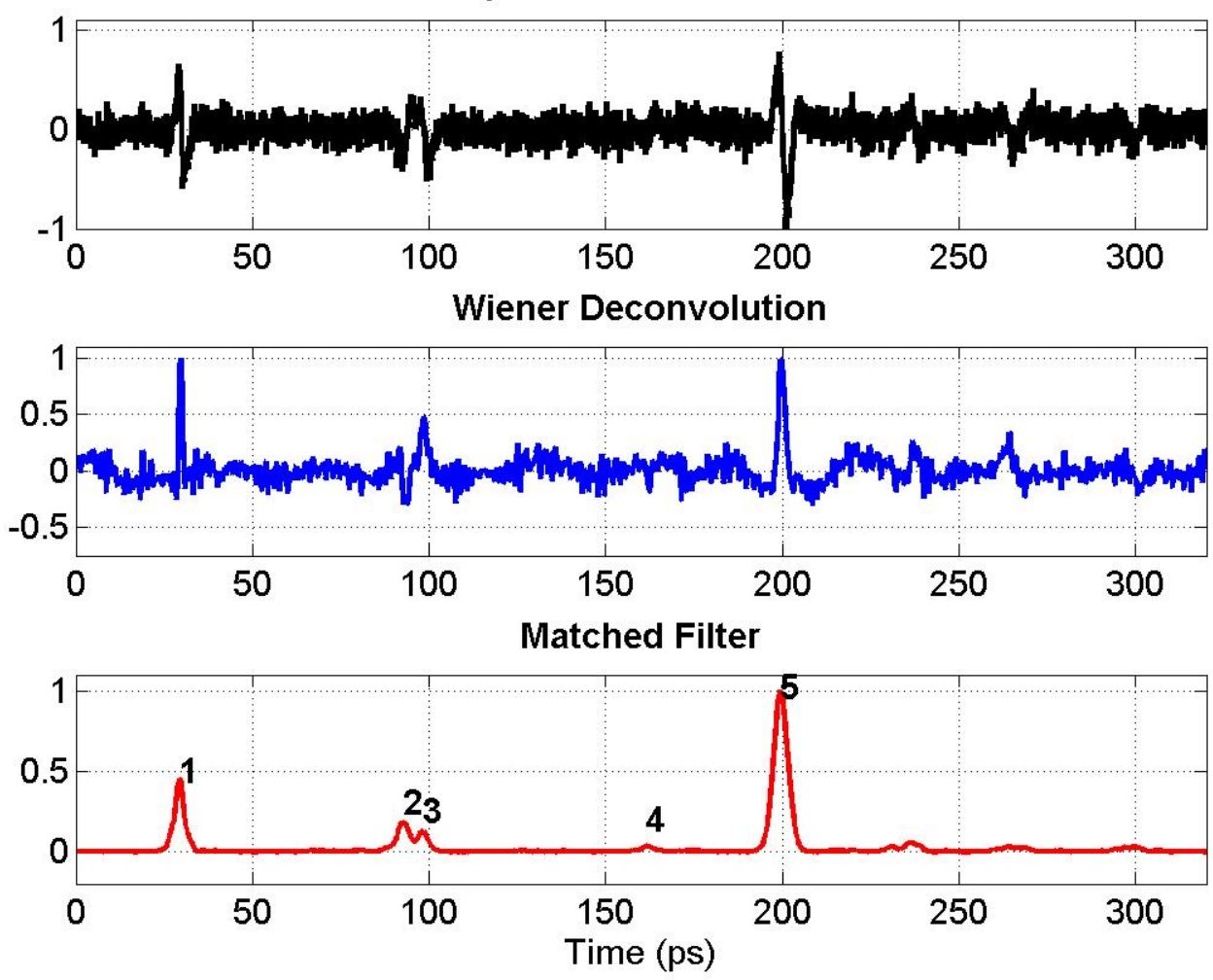

Figure 4.1.5: Comparison of results when $d_{2}=0.70 \mathrm{~mm}$. THz waveform reflected from multiple layers of planar media (top panel), the same waveform after processing with the Wiener deconvolution filter (middle panel) and after processing with the matched filter (bottom panel). The matched filter provides peaks with superior SNR. The numbers near each peak correspond to the circled boundary numbers in Figure 4.1.3. Each of the waveforms was normalized for comparison.

\subsubsection{THz Image Processing Results: B-Scans}

Image data was collected by raster scanning over the sample for the case when $d_{2}=0.70 \mathrm{~mm}$. Figure 4.1.6 shows the unprocessed $\mathrm{THz}$ B-scan for a slice through the center of the sample. The columns were sampled at $1 \mathrm{~mm}$ increments and are plotted along the horizontal axis (labeled distance). For each column, the vertical 
depth into the sample is indicated by the delay time along the vertical axis. Thus, the top of the figure is above the sample, and each vertical column in the B-scan image corresponds to an A-scan similar to the one shown in the top panel of Figure 4.1.5. Therefore, the boundaries between the layers are indicated by horizontal lines in the B-scan image. As expected, horizontal lines appear at approximately 29 ps, 92 ps, 98 ps, 162 ps, and 199 ps, corresponding to reflections from boundaries 1, 2, 3, 4 and 5, respectively, in Figure 4.1.3. The lines corresponding to boundaries 2 and 3 are very close together due to the small air gap $\left(d_{2}=0.70 \mathrm{~mm}\right)$ in this scenario. The reflected pulse at boundary 4 (appearing at $162 \mathrm{ps}$ ) is relatively faint due to the attenuation power lost in the reflection from boundaries 1,2 and 3, as well as the absorption within the two acrylic layers. Boundary 5 is relatively strong due to the high reflection coefficient between air and the metal conductor. Horizontal lines appearing with delay beyond 199 ps are due to multiple reflections from the layers above the metal substrate.

The layer boundaries are relatively difficult to distinguish in the unprocessed B-scan image (Figure 4.1.6) due to poor SNR. Figure 4.1.7 shows the B-scan for the conventional Wiener deconvolution processing, where each column in the B-scan image is similar to the middle panel of Figure 4.1.5. The boundary layers in Wiener deconvolution results are somewhat easier to identify visually in the B-scan data (as compared to the A-scan in the middle panel of Figure 4.1.5) because the eye can naturally detect the correlation as it scans horizontally across the image. This is similar to the SNR gain that would be achieved by averaging many A-scan waveforms. However, the weaker reflections (such as from boundary 4 at 162 ps) are still relatively difficult to detect due to poor SNR. 
Finally, Figure 4.1.8 shows the B-scan after matched filtering based on the MLE. The horizontal lines in the matched filter image appear as brighter lines above a dark black background due to the superior SNR after processing with this method. As expected, the lines are thicker than those in the Wiener deconvolution data for the same reason that the peaks in the A-scan data are wider in Figure 4.1.5. The horizontal line at 162 ps (due to boundary 4 in Figure 4.1.3) is faint in this image due to the large dynamic range between this pulse and the reflected pulse from the metal plate (boundary 5) at 199 ps. This peak is more visible in the A-scan data in the bottom panel of Figure 4.1.5.

The following section will use ROC curves to perform a qualitative comparison of the results of the Wiener deconvolution results and the matched filter, by attempting to detect boundary 4, which appears near 162 ps.

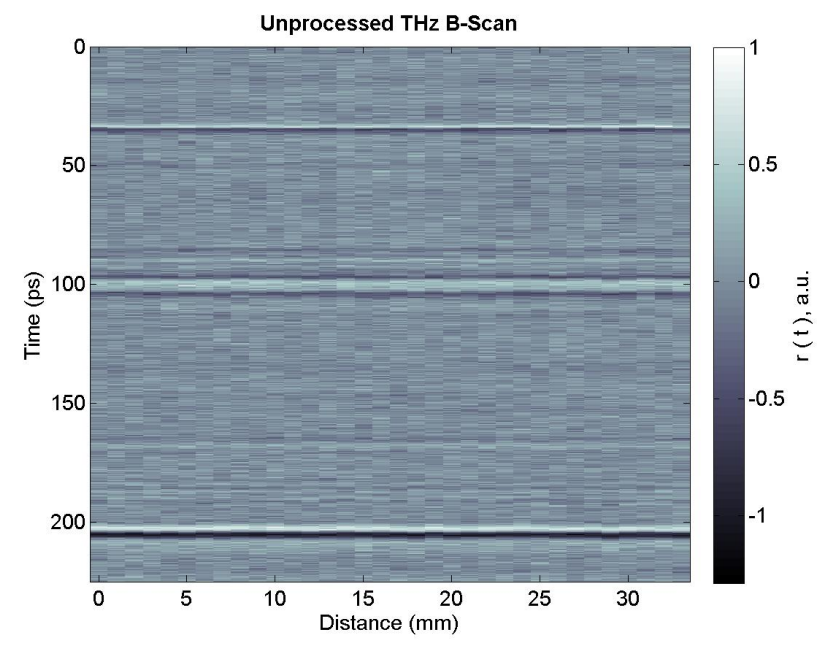

Figure 4.1.6: B-scan slice through the center of the unprocessed $\mathrm{THz}$ sample data illustrated in Figure 4.1.3 for the case when $d_{2}=0.70 \mathrm{~mm}$. Each column in the B-scan data is similar to the A-scan data shown in the top panel of Figure 4.1.5, and therefore the boundaries between the layers are indicated by horizontal lines in the B-scan images. 


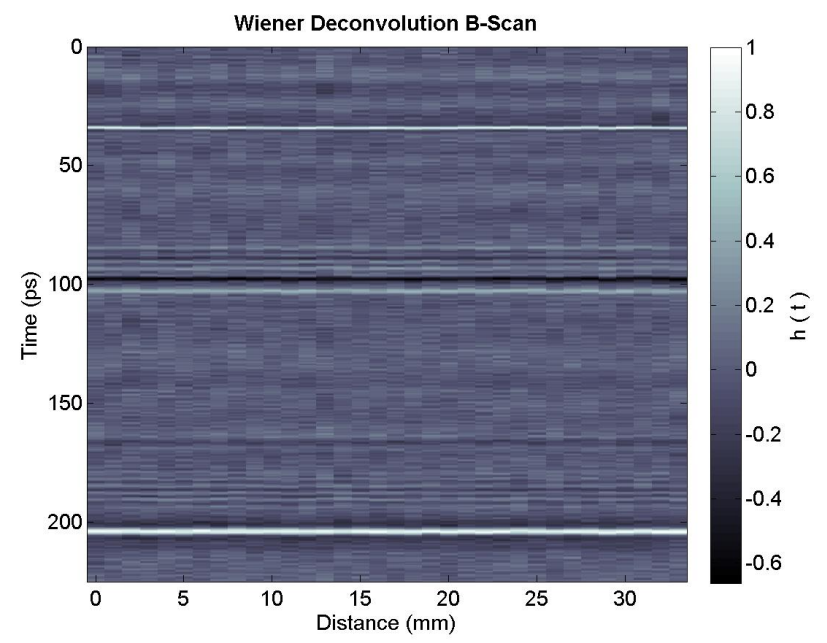

Figure 4.1.7: B-scan slice through the center of the sample illustrated in Figure 4.1.3 for the case when $d_{2}=0.70 \mathrm{~mm}$ from the $\mathrm{THz}$ data processed with Wiener deconvolution. Each column in the B-scan data is similar to the A-scan data shown in the middle panel of Figure 4.1.5, and therefore the boundaries between the layers are indicated by horizontal lines in the B-scan images.

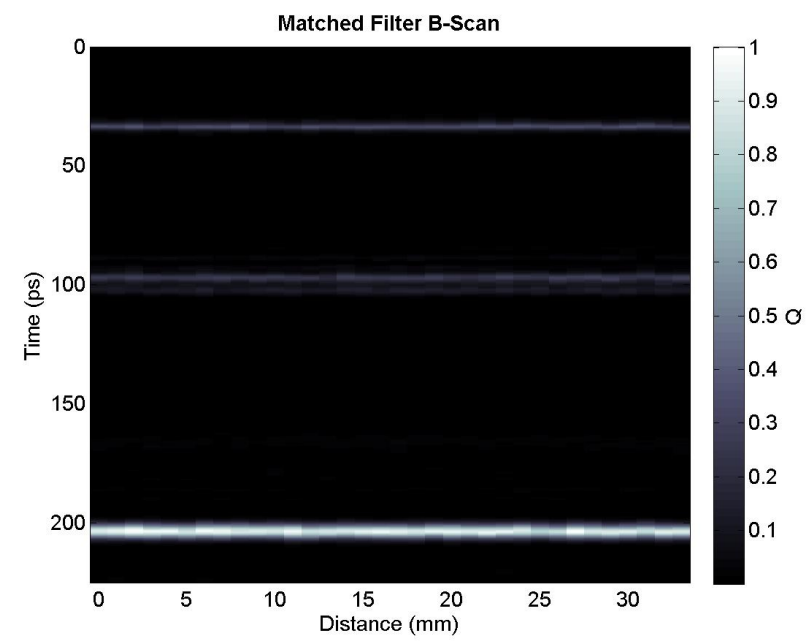

Figure 4.1.8: B-scan slice through the center of the sample illustrated in Figure 4.1.3 for the case when $d_{2}=0.70 \mathrm{~mm}$ from the $\mathrm{THz}$ data processed with a matched filter. Each column in the B-scan data is similar to the A-scan data shown in the bottom panel of Figure 4.1.5, and therefore the boundaries between the layers are indicated by horizontal lines in the B-scan images. 


\subsubsection{Receiver Operating Characteristic (ROC) Curves}

The previous section showed $\mathrm{THz}$ B-scans for the case when $d_{2}=0.70 \mathrm{~mm}$. A qualitative comparison of those images indicated that the layer boundaries were easier to distinguish in the matched filter images. In this section, a quantitative comparison of these same results is made using ROC curves. Here, the goal is to detect one of the weakly reflected pulses. In particular, the reflection from boundary 4 (see Figure 4.1.3) which appears near $162 \mathrm{ps}$ is selected due to its relatively weak signal strength compared to the background noise in each image. For the purposes of this analysis, the absolute value of the Wiener deconvolution data was used. Additional sample waveforms, similar to the one shown in Figure 4.1.5 were collected by raster scanning over the sample for the case when $d_{2}=0.70 \mathrm{~mm}$. As in the case Figure 4.1.5, the shortest possible integration time, $10 \mathrm{~ms}$ per sample measurement, was used to collect the sample waveform at each position.

Since the objective is to detect the presence of boundary 4 , an 8 ps time window centered at 162 ps was selected for each set of image data. The peak values within this time window for the Wiener deconvolution data and the matched filter data are shown in the red histograms in the left and right panels, respectively, of Figure 4.1.9. For comparison, the peak values within an 8 ps time window at the very beginning of each data set were selected. The data within this time window are due only to noise because it precedes reflections from boundary 1 of the stratified sample. The peak values within the 0 - 8 ps time window for the Wiener deconvolution data and the matched filter data are shown in the blue histograms in the left and right panels, respectively, of Figure 4.1.9. As expected, there is significantly more overlap between the histograms for the Wiener deconvolution data. 
Sweeping a threshold parameter across the horizontal axis of each histogram data set in Figure 4.1.9 produced the ROC curves in Figure 4.1.10. As expected, the curves indicate an improvement in detection with the proposed matched filter method, based on the MLE performance over the Wiener deconvolution method.

Figure 4.1.11 shows the performance improvement that is gained when increasing the averaging time to 10 waveforms per pixel during the data collection. The greater separation between the histograms results in both ROC curves showing better detection performance. This indicates that the Wiener deconvolution method can adequately detect weak reflections, if there is sufficient integration time during the data collection. However comparison of the two data sets shows that the matched filter method is capable of providing excellent detection performance with minimal integration time - only a single waveform per pixel.

The performance improvement gained by matched filter processing means that $\mathrm{THz}$ NDE imaging data could be collected much more quickly, providing a significant reduction in the imaging time needed for medical imaging in hospitals, security screening at airports or other checkpoints, and product evaluation on assembly lines in factories. Such an improvement is expected to advance the deployment of $\mathrm{THz}$ scanning equipment for practical applications. 

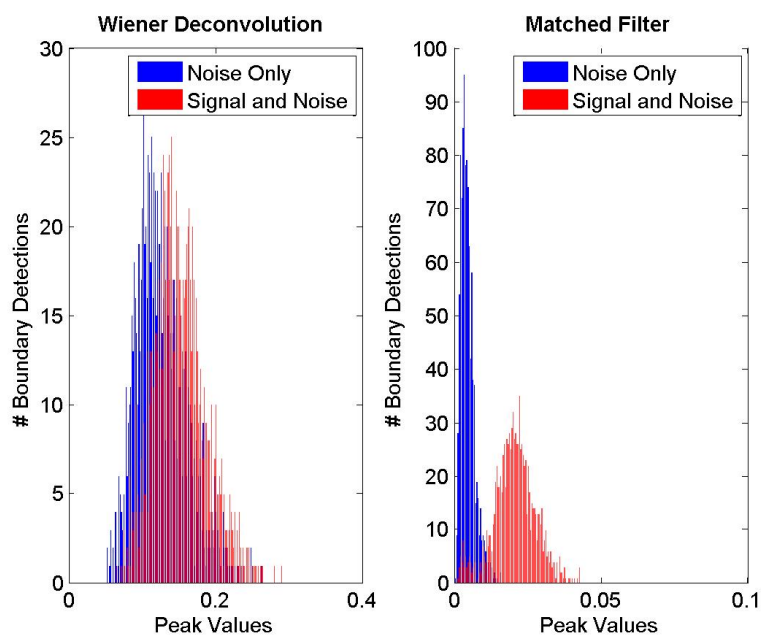

Figure 4.1.9: Histograms show the peak signal detection within a signal and noise time windows for the sample in Figure 4.1 .3 when $d_{2}=0.70 \mathrm{~mm}$. (Left panel.) The signal and noise histograms for the Weiner deconvolution data have a relatively wide region of overlap because the SNR in the signal time window is poor. (Right Panel.) The signal and noise histograms for the matched filter data show a smaller region of overlap because the SNR is better. Sweeping a threshold parameter across the horizontal axis of each data set produces the ROC curves in Figure 4.1.10.

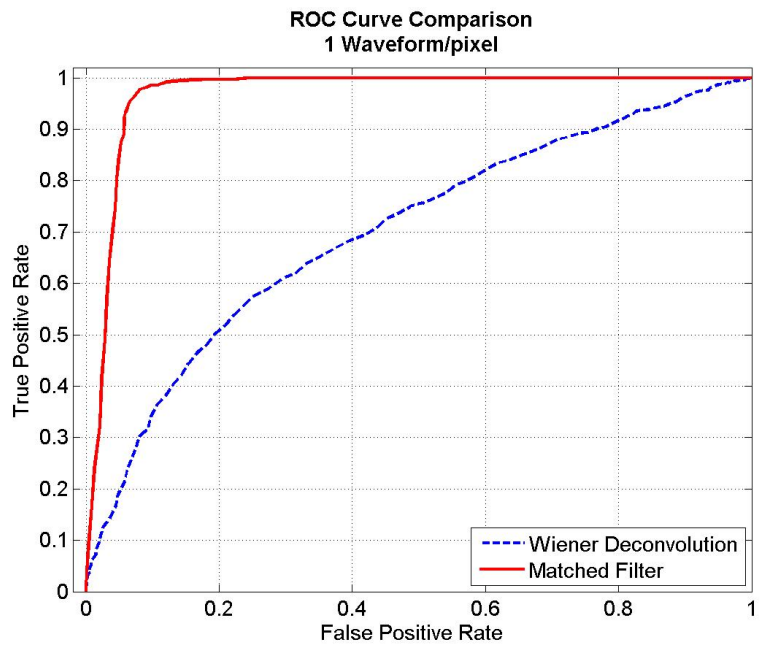

Figure 4.1.10: ROC curves for the Wiener deconvolution and matched filter data shown in Figure 4.1.9. The curves indicate detection with the proposed matched filter method, based on the MLE, is almost ideal while the detection performance of the Wiener deconvolution method is relatively poor. 


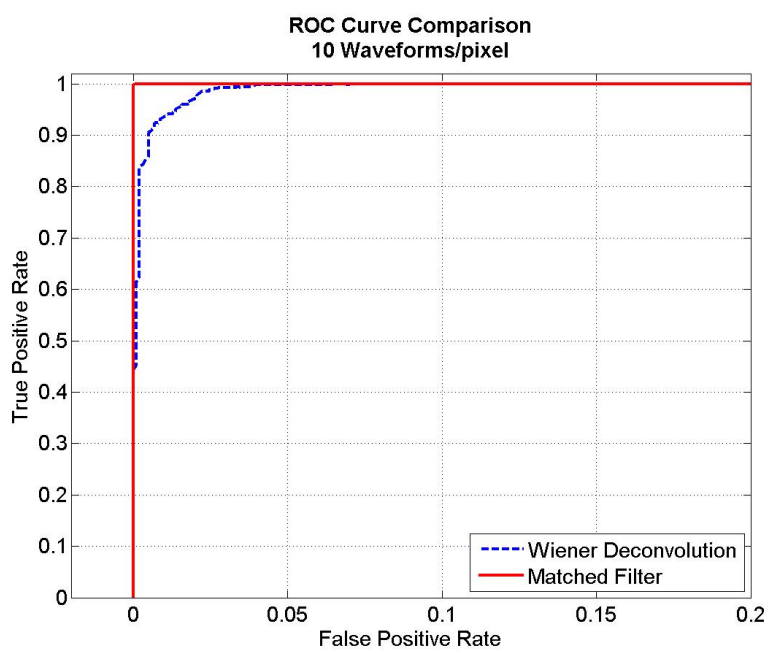

Figure 4.1.11: ROC curves similar to those in Figure 4.1.10, show detection performance increases when the averaging per pixel is increased from 1 waveform to 10 waveforms. The performance of the Wiener deconvolution method is improved significantly by averaging, but at the expense of a $10 \mathrm{X}$ increase in integration time during data collection. The performance of the matched filter detector is virtually ideal.

\subsection{THz NDE of Thin Films of Air in Polycarbonate}

Experimental results in the previous section demonstrated how THz TDS systems can be used for NDE of thick layered media, but challenges arise when then the delay spread between multipath arrivals is less than the pulse width. For example, the sample configuration shown in Figure 4.1 .3 with $d_{2}=0.70 \mathrm{~mm}$, the signal processing results shown in Figure 4.1.5 indicate that the THz echo pulses from boundaries 2 and 3 are nearly overlapping one-another. Thus, for thin layers, the Weiner Deconvolution and Matched Filtering approaches that were demonstrated in the previous section cannot be applied.

In this section, the MFP approach from Section 3.3 is used to demonstrate THz NDE for thin film samples. The thickness of thin films is extracted from $\mathrm{THz}$ measure- 
ments of thin film samples composed of polycarbonate in air background. The MFP algorithm is used with the MSE objective function comparing measured $\mathrm{THz}$ waveforms with simulated replica waveforms in the time domain. In addition, the MFP algorithm is used with the Bartlett objective function comparing the corresponding measured $\mathrm{THz}$ spectrum with replica spectra in the frequency domain. The process is repeated to measure a variety of thin film thicknesses in each case and the thickness estimates from each objective function are compared with one another and with independent measurements made with a Vernier caliper.

This section describes $\mathrm{THz}$ experiments that use the MFP approach for NDE of a polymer that contains an air film below the surface. Thus, the examples considered here could be representative of industrial NDE applications which require detection and evaluation of defects (e.g. air bubbles) below the surface of the sample under test.

In this study, the material properties (frequency-dependent index of refraction and extinction coefficient) of the polymer are available from a separate transmission measurement, and the objective is to estimate the depth of an air gap (located below the surface of the polymer) as well as the thickness of the air gap. Several possible depths for the air gap are considered here. When the air gap is at a shallow depth, the surface layer is also a thin film. Thus, the thicknesses of a stack of three layers (calibration layer, thin polymer layer and thin air gap) are all estimated simultaneously with $\mathrm{THz}$ MFP. 


\subsubsection{Terahertz Measurement System}

The experiments discussed here were performed in the Northwest Electromagnetics and Acoustics Research Laboratory (NEAR-Lab) at Portland State University (PSU) with a Picometrix T-Ray $4000 \mathrm{THz}$ time-domain spectroscopy (TDS) system from Advanced Photonics, Inc. Details about the THz-TDS system are available in [37]. The THz-TDS system was configured for monostatic measurements at normal incidence as illustrated in Figure 4.2.1.

The T-Ray 4000 measurement system consists of an enclosed unit that contains a femto-second (fs) laser and variable delay module for controlling the delay on the probe beam of the THz TDS system. The laser pulse is split into a pump and probe path which exits the main control unit via separate fiber optic cables to a photoconductive switch in $\mathrm{THz}$ transmit and receive module, respectively. In rapid scan mode, the T-Ray 4000 acquires a single 320 ps THz waveform in 10 ms. The number of $\mathrm{THz}$ waveforms averaged for each measurement can be increased to improve the SNR at the expense of increased integration time.

Three types of THz TDS measurements were performed: a background measurement, a reference measurement and the sample measurements. The background waveform was recorded with nothing in the optical path and therefore contains only the echo pulses within the collinear head, which are common to both the reference and sample measurements. Thus, the background waveform was subtracted from both the sample and reference waveforms before either processing method was employed. The reference measurement was taken with a gold mirror in place of the sample. For both background and reference measurements, 10,000 waveforms were averaged to increase 


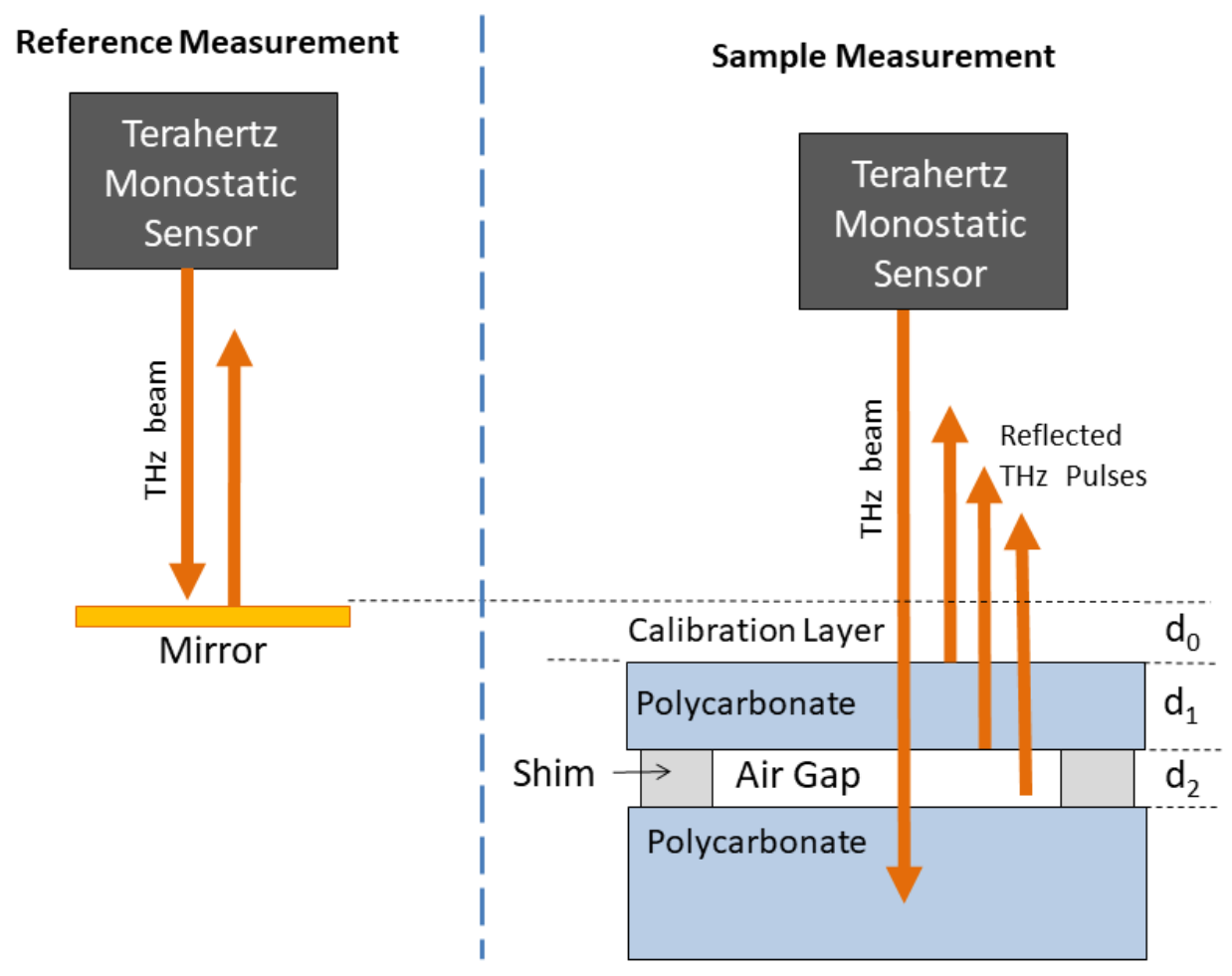

Figure 4.2.1: Illustration of the $\mathrm{THz}$ NDE experiment configuration (not to scale). Left: Measurement configuration for the $\mathrm{THz}$ reference, which is used to approximate the $\mathrm{THz}$ source signal in MFP processing. Right: Measurement configuration for the layered sample under test. A calibration layer (air) with unknown thickness, $d_{0}$, accounts for the offset distance between the reference mirror and the surface of the sample. A shim (Scotch Removable Double Sided Tape) located a distance of $d_{1}$ below the sample surface creates an air gap with thickness, $d_{2}$. THz MFP is used to estimate the thicknesses of all three layers $\left(d_{0}, d_{1}\right.$, and $\left.d_{2}\right)$, simultaneously. 
the SNR. For THz NDE applications, it is often desired to quickly record sample measurements with low integration time and process the data with a single reference measurement that is typically recorded with higher SNR (longer integration time).

As discussed in Section 3.3.3, THz MFP in reflection configuration requires the $\mathrm{THz}$ source signal to be approximated with a reference measurement from a mirror. The left side of Figure 4.2.1 shows the configuration for the $\mathrm{THz}$ reference measurement from a gold mirror. A total of 10,000 reference waveforms were averaged to maximize the SNR. After performing a DFT, the reference spectrum could be used to approximate the source spectrum, $D(f)$, needed to generate the replica spectra, $R_{r}(f, \boldsymbol{a})$, in Eq. 3.3.7.

\subsubsection{Layered Media Samples}

The right side of Figure 4.2.1 illustrates the measurement configuration for the sample under test, which consisted of a small air gap between two layers of polycarbonate. The polycarbonate layers were homogeneous with smooth parallel surfaces, making them ideal for a variety of layer configurations for $\mathrm{THz}$ laboratory measurements. In practice, there is always a small offset distance between the surface of the sample under test and the surface of the mirror during the reference measurement. This small offset can be accounted for with MFP by including an additional thin layer of air above the sample's surface when generating replica spectra with the propagation model [30]. In the remainder of this dissertation this hypothetical layer will be referred to as the calibration layer, denoted $d_{0}$. The air gap between two layers of polycarbonate was created using a shim (Scotch Removable Double Sided Tape 667, Cat. 238) with a thickness of 2.4 mils $(61 \mu m)$, per the manufacturer. 
The depth of the air gap below the polycarbonate surface is $d_{1}$. Four different samples thicknesses for layer $d_{1}$ were tested using polycarbonate film samples obtained from Tap Plastics, Inc. with thicknesses of 10,15, 20 and 30 mils $(1$ mil $=1 / 1000$ inch $=25.4 \mu \mathrm{m})$. Each of the samples were measured with a digital Vernier caliper with 10 $\mu m$ resolution and $20 \mu m$ accuracy. The average of 10 measurements for each sample film are recorded in Table 4.2.1.

Table 4.2.1: Four samples of thin polycarbonate films (layer $d_{1}$ in Figure 4.2.1) were evaluated in this study.

\begin{tabular}{|c|c|c|}
\hline Sample & $\begin{array}{c}\text { Manuf. Spec. } \\
(\mathrm{mils})\end{array}$ & $\begin{array}{c}\text { Vernier Cal. } \\
(\mu \mathrm{m})\end{array}$ \\
\hline A & $30 \mathrm{mil}$ & 740 \\
\hline B & $20 \mathrm{mil}$ & 520 \\
\hline C & $15 \mathrm{mil}$ & 390 \\
\hline D & $10 \mathrm{mil}$ & 260 \\
\hline
\end{tabular}

For the THz NDE example discussed in this dissertation, the objective is to estimate the thickness of layers within a known medium, a propagation model is used to generate replica spectra for various possible layer thicknesses, and MFP is used to compare the replicas with measurements. The propagation modeling required to make replicas for the THz NDE experiment requires a priori knowledge of the index of refraction for the sample materials.

A THz measurement was performed in transmission configuration with a single layer of polycarbonate (sample A in Table 4.2.1) to extract the material properties of polycarbonate. The THz TDS signal processing methods for extraction of material properties from solid materials are already documented in the literature [3], [8] and therefore will not be discussed in detail here. 

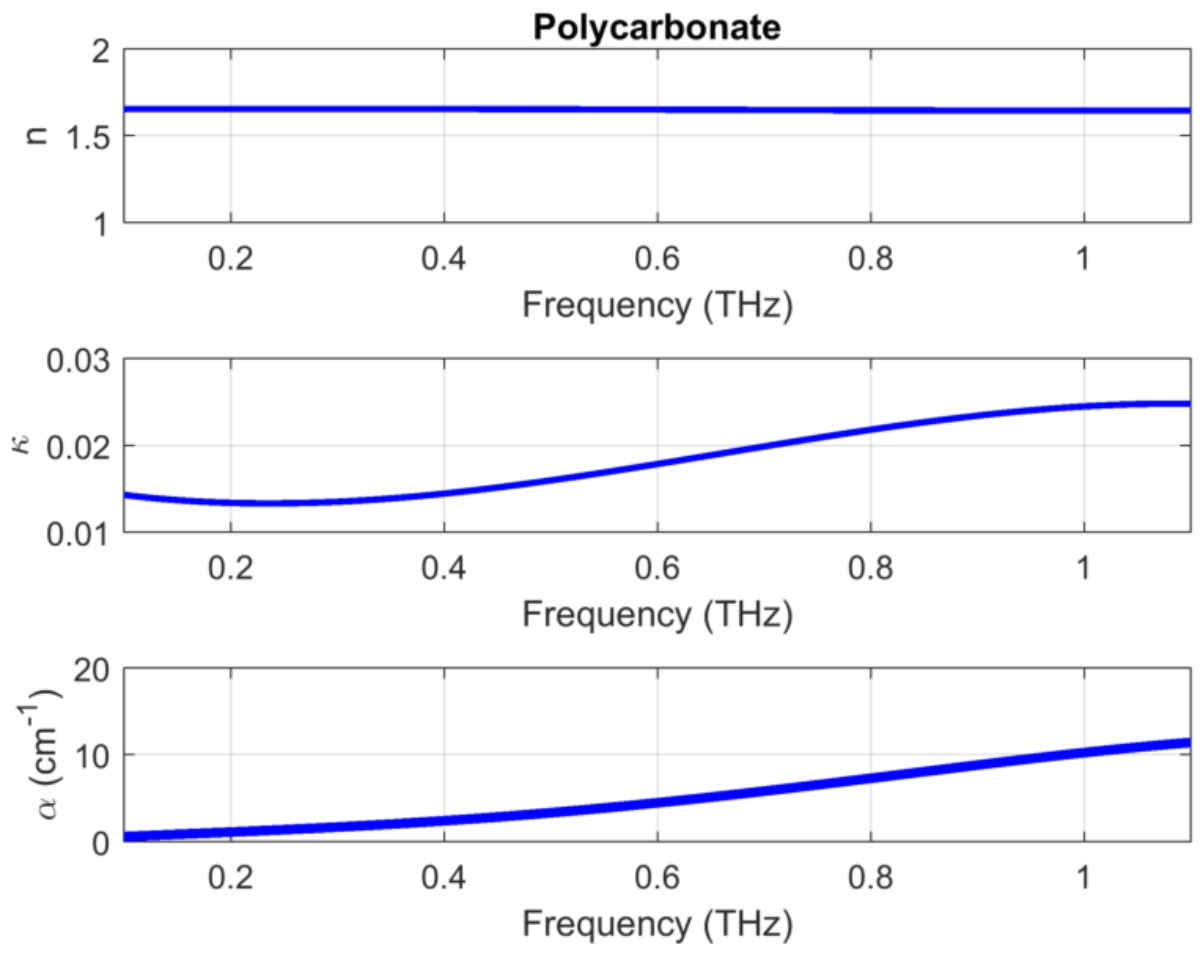

Figure 4.2.2: Complex index of refraction for Polycarbonate were calculated from $\mathrm{THz}$ measurement data. Top panel: real part of the refractive index, $n$. Middle Panel: Extinction coefficient, $\kappa$. Bottom Panel: Absorption coefficient $\alpha$. Results are consistent with data in the literature [8], [60].

The resulting complex index of refraction and absorption coefficient are shown in Figure 4.2.2, which is consistent with data in the literature [8], [60].

For the polycarbonate sample the group index is $n_{g}=1.60$. Therefore, the range resolution in polycarbonate is $467 \mu m$, and samples $C$ and $D$ in Table 4.2 .1 are thin films.

The following section will show that $\mathrm{THz}$ MFP can be used to accurately estimate the thickness of both the thick and thin layers in Table 4.2.1. 


\subsubsection{Measurement Data Processing}

The monostatic THz sensor configuration shown in Figure 4.2.1 was used to record $\mathrm{THz}$ waveforms above sample A from Table 4.2.1. A total of 300 waveforms were each recorded at a single position with minimal measurement integration time (10 $\mathrm{ms}$ (waveform). The waveforms were truncated within a time window with duration of $60 \mathrm{ps}$ surrounding the reflected pulses from the surface and the air gap below the surface.

The top panel of Figure 4.2.3 shows the mean THz waveform for sample A. Note that reflected THz pulse from the top surface of polycarbonate layer arrives at approximately 16 ps. The $\mathrm{THz}$ pulses from the upper and lower boundaries of the air gap overlap with one another resulting in a single peak at approximately $24.5 \mathrm{ps}$. Thus, thickness of $d_{2}$ layer cannot be estimated from a simple time difference of arrival (TDOA) analysis based on impulse response or matched filter analysis as discussed in Section 4.2.2.

The middle plot of Figure 4.2.3 shows the spectrum of the mean waveform in the top panel, truncated with the bandwidth of $0.1-1.3 \mathrm{THz}$. The spectrum shows peaks and nulls separated by approximately $116.5 \mathrm{GHz}$, due to the multipath delay of approximately $\left(1 /\left(116.5 \times 10^{9}\right)=8.5\right.$ ps between the $\mathrm{THz}$ pulse from the polycarbonate surface and the $\mathrm{THz}$ pulses from the air gap.

An FFT was performed on the each of the truncated THz waveforms and the 300 resulting spectra were truncated within the bandwidth of $0.1-1.3 \mathrm{THz}$ for maximum SNR. Each of the spectra were normalized to unit vectors as discussed in Sections 3.3.4 and 3.3.5. This normalization is not necessary for MFP, but provides a maximum 
possible output of unity in the Bartlett processor if the replica weight vectors are also normalized to unit vectors [45], [49]. The covariance was then estimated using Eq. 3.3 .9 with $L=145$ frequency bins and $Z=300$ snapshots. The resulting covariance matrix is a $L \times L$ square matrix of complex values. The bottom panel of Figure 4.2.3 shows the absolute value of the covariance matrix on a $\mathrm{dB}$ scale.

Several features of the covariance matrix in Figure 4.2 .3 can be observed. Higher levels of covariance appear for the covariance between low frequency bins in the upper left corner of the covariance matrix due to the higher spectral levels at low frequencies of the measured $\mathrm{THz}$ spectra. Conversely, the lower right corner of the covariance matrix shows relatively low covariance between high frequency bins because of the lower spectral levels at the high end of the $\mathrm{THz}$ spectrum. Stronger covariance is observed along the diagonal of the covariance matrix due to the high level of autocovariance for each frequency bin, relative to adjacent bins. The covariance matrix is symmetric about the diagonal because the magnitude of the covariance between each pair of frequency bins is the same. Finally, we note that the peaks and nulls observed in the THz spectrum (middle panel of Figure 4.2.3) are also evident at 116.5 $\mathrm{GHz}$ intervals in the covariance matrix due to the high level of covariance around the peaks in the spectra.

Data was processed for the other samples $(B-D)$ in a manner similar to the method discussed for Sample A above. Figure 4.2.4 shows the processing results for Sample $\mathrm{D}$, which had the thinnest surface layer, $d_{1}$, of all the samples.

The top panel of Figure 4.2.4 shows the mean THz waveform for sample D, where the reflected $\mathrm{THz}$ pulse from the top surface of polycarbonate layer arrives at approximately 16 ps. However, due to the thin layer of polycarbonate in Sample D, the THz 

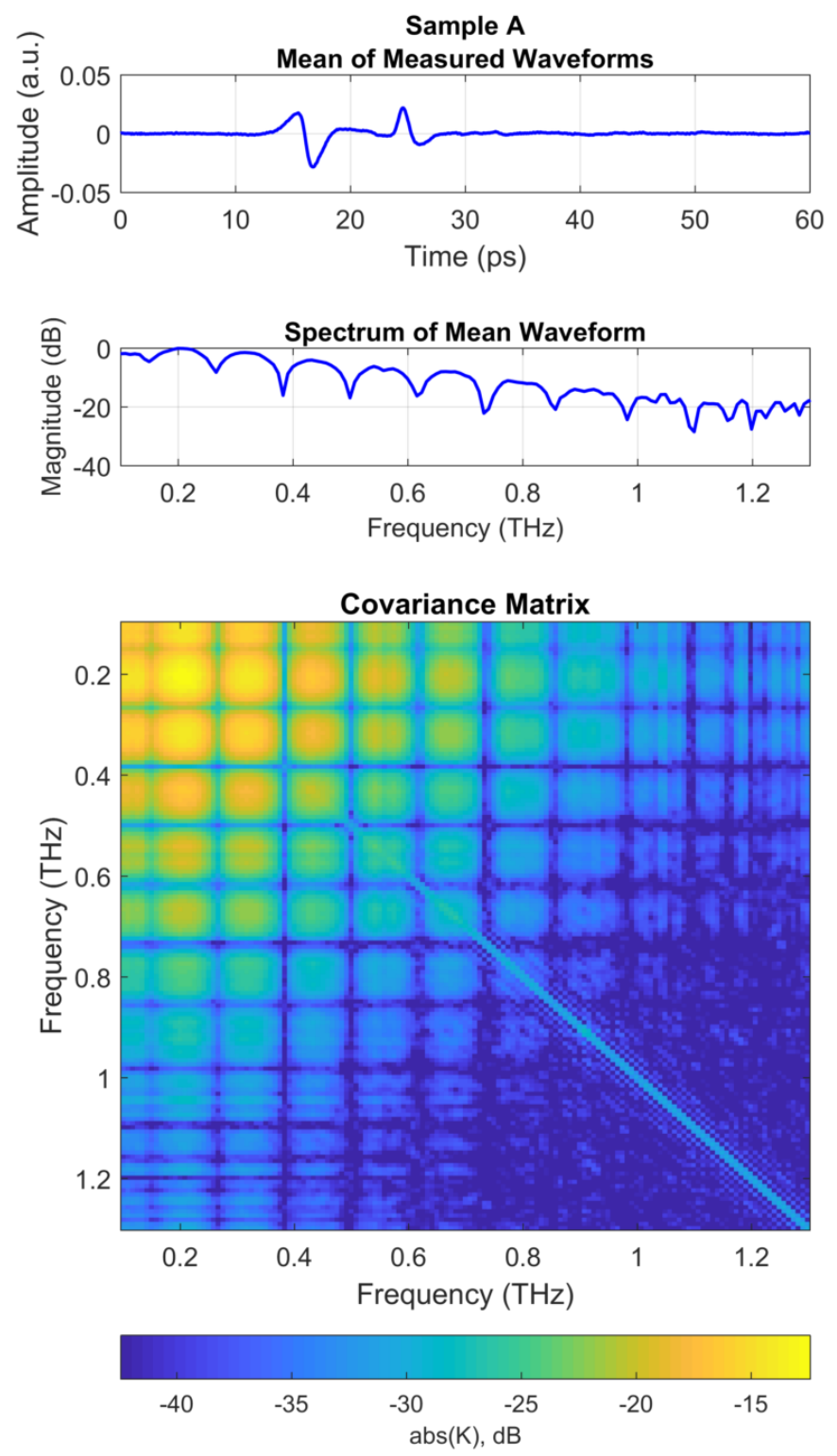

Figure 4.2.3: Measured data for Sample A. Top Panel: Mean of measured THz waveforms. Note that reflected $\mathrm{THz}$ pulse 1 (illustrated in Figure 4.2.1) arrives at approximately $15 \mathrm{ps}$, and pulses 2 and 3 overlap with one another at approximately 25 ps. Middle Panel: Spectrum of mean waveform in the top panel. Bottom Panel: Covariance matrix computed with Eq. 3.3.9 using the spectrum of each 300 measured waveform. 
pulses from the lower surface of the polycarbonate layer overlaps with the $\mathrm{THz}$ pulses reflected from the upper and lower boundaries of the air gap resulting in a single distorted waveform between $13-21$ ps. Thus, thickness of layers $d_{1}$ and $d_{2}$ cannot be estimated from a simple TDOA analysis. The following section will demonstrate that $\mathrm{THz}$ MFP approach discussed in Section 3.3.1 can be used to accurately estimate the thickness of both of these layers.

The middle panel of Figure 4.2.4 shows the spectrum of the mean waveform in the top panel, truncated with the bandwidth of $0.1-1.3 \mathrm{THz}$. The spectrum shows peaks and nulls separated by approximately $333 \mathrm{GHz}$, due to the multipath delay of approximately $\left(1 /\left(333 \times 10^{9}\right)=3\right.$ ps between the $\mathrm{THz}$ pulse from the polycarbonate surface arriving at $16 \mathrm{ps}$ and the subsequent overlapping $\mathrm{THz}$ pulses arriving at 19 ps.

The covariance matrix for Sample D was generated in a manner similar to that used for Sample A, above. The bottom panel of Figure 4.2.4 shows the absolute value of the covariance matrix for Sample D. Note, that the covariance matrix shows similar features to that of the covariance matrix for Sample A (bottom panel of Figure 4.2.3) except that peaks and nulls are observed at intervals of approximately $333 \mathrm{GHz}$, due to the shorter delay time between the arrival of multipath pulses in this sample as discussed above.

\subsubsection{Generating Replica Spectra}

As discussed in Section 3.3, the MFP approach compares the covariance matrix with a set of modeled replica fields. The transfer matrix model discussed in Appendix 

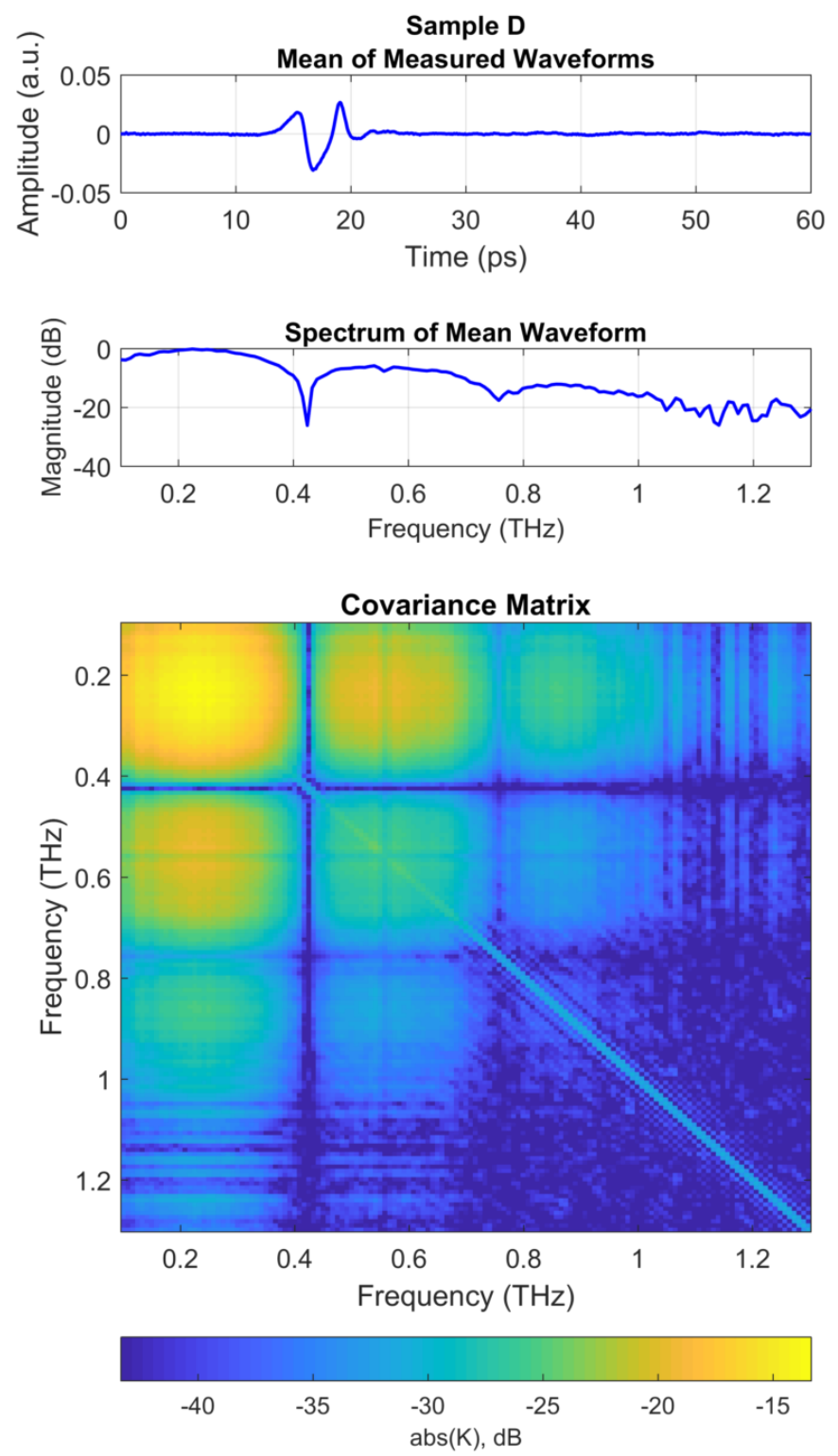

Figure 4.2.4: Measured data for Sample A. Top Panel: Mean of measured THz waveforms. Note that reflected THz pulses 1, 2 and 3 (illustrated in Figure 4.2.1) all overlap on one another. Middle Panel: Spectrum of mean waveform in the top panel. Bottom Panel: Covariance matrix computed with Eq. 3.3.9 using the spectrum of each 300 measured waveform. 
A was used to generate a set of transfer functions, $H_{s}(f, \boldsymbol{a})$, for the layered media sample illustrated in Figure 4.2.1, where the parameter vector, $\boldsymbol{a}$, contained the thicknesses of the three layers, i.e. $\boldsymbol{a}=\left[d_{0}, d_{1}, d_{2}\right]^{T}$. A range of possible thicknesses in were considered for each of the three layers. A total of 51 thicknesses from $0-$ $500 \mu \mathrm{m}$ in $10 \mu \mathrm{m}$ intervals were considered for the calibration layer, $d_{0}$, and a total of 101 thicknesses from $0-1000 \mu m$ in $10 \mu m$ intervals were considered for both the polycarbonate layer, $d_{1}$, and air gap layer, $d_{2}$.

The index of refraction (Eq. A.2.3) used for the calibration layer and the air gap between the polycarbonate layers was $n=1$ for all frequencies. Thus, the absorption spectra of the air was neglected because it is expected to have negligible impact on the measured spectra for these thin layers with thicknesses on the order of a few hundred microns. The frequency-dependent index of refraction for the polycarbonate layers was taken from a separate transmission measurement as discussed in Section 4.2 .2 .

It is important to note that the propagation model in Appendix A is a frequencydomain model that computes the transfer function for the layered stack, $H_{s}(f, \boldsymbol{a})$, at finite frequency bins, and Fourier synthesis is required to estimate the impulse response for the sample layers. This is because the model assumes an infinite number of multipath reflections within the sample layers whereas the THz measurement system truncates mutlitpath reflections outside a measurement window of finite duration (320 ps for the T-Ray 4000 used in this study). Therefore, to avoid aliasing of the multipath arrivals in the time-domain, a frequency resolution (four times smaller than the resolution of the measurement data) was used to generate the simulated spectra, $H_{s}(f, \boldsymbol{a})$. 
Several signal processing steps were required to compute replicas of the received spectra. The transfer functions were generated for positive frequencies using the propagation model and then zero-padded to create the analytic spectrum. An inverse FFT was then performed to get the analytic signal in time domain, which was converted to a real signal (impulse response). This real signal was was then interpolated onto the same time axis as the truncated measured waveforms in order to eliminate multipath arrivals in the model that would be outside the measurement time window of the THz TDS system. An FFT was then performed on the resulting time domain impulse response (similar to the FFT performed on the measured data), and the resulting spectrum was multiplied by the $\mathrm{THz}$ source spectrum $D(f)$ for each transfer

function, $H_{s}(f, \boldsymbol{a})$, as discussed in Section 3.3.4, to give the replica spectra for the received THz spectra, $R_{\text {sim }}(f, \boldsymbol{a})$. Finally, the normalized weight vectors were computed using Eq. 3.3.8. Due to the similarity of the samples $(A-D)$, the process of computing the replica spectra only needed to be computed once. The resulting set of replicas were stored in a file and loaded for comparison with each of the covariance matrix for each sample $A-D$ in this study.

\subsubsection{THz MFP Results for Sample A}

The covariance matrix in Figure 4.2.3 was compared with the replica weight vectors (discussed in section 3.3.4) using the Bartlett processor and the MV processor using Equations 3.3.11 and 3.3.12, respectively. The output, $P(\boldsymbol{a})$ of each processor is a scalar value for each possible parameter vector, $\boldsymbol{a}$. Thus, parameter vector corresponding to the maximum value of the processor output is taken to be the the best estimate, $\hat{\boldsymbol{a}}$, for the unknown parameters. 
For Sample A, the best estimate of the parameter vector from the Bartlett processor was $\hat{\boldsymbol{a}}_{\boldsymbol{B}}=[190,750,70]^{T} \mu m$, and the best estimate of the parameter vector from the MV processor was $\hat{\boldsymbol{a}}_{M V}=[180,750,70]^{T} \mu m$. Thus, the estimated thickness of the calibration layer was nearly the same for both processors and accurate to within the measurement resolution of the Vernier caliper and the replicas that were generated $(10 \mu m)$.

As discussed in Section 3.3.7, ambiguity surfaces are often used in the MFP literature to evaluate the ambiguity around the estimated parameters. For Sample A, a $2 D$ image of the ambiguity surface for the Bartlett processor output was generated for $d_{0}$ vs. $d_{1}$ by selecting the ambiguity data for which the $d_{2}=\hat{d}_{2, B}$ and organizing the remaining ambiguity data into the $2 D$ image shown in the top panel of Figure 4.2.5. Thus, each pixel in the image gives the ambiguity of the Bartlett processor corresponding to the replica spectrum that was computed with the values of $d_{0}$ and $d_{1}$ given by the horizontal and vertical axes of the image, respectively. The ambiguity surface for the Bartlett processor shows relatively high ambiguity surrounding the global maximum, located at coordinates $\hat{d}_{0, B}=190 \mu \mathrm{m}$ and $\hat{d}_{1, B}=750 \mu \mathrm{m}$.

It is important to note that the ambiguity data shown in the top panel of Figure 4.2.5 was normalized by the peak ambiguity value. Therefore, the color axis for the ambiguity image spans the range between minimum and maximum possible values of $[0-1]$. Each of the ambiguity surfaces presented in this dissertation are normalized in the same way, which allows for direct comparisons of the ambiguity data from the Bartlett and MV processors for each sample.

A $2 D$ image of the MV ambiguity surface was generated for $d_{0}$ vs. $d_{1}$ of Sample A, as shown in the bottom panel of Figure 4.2.5. As noted in Section 3.3.6.2, the MV 

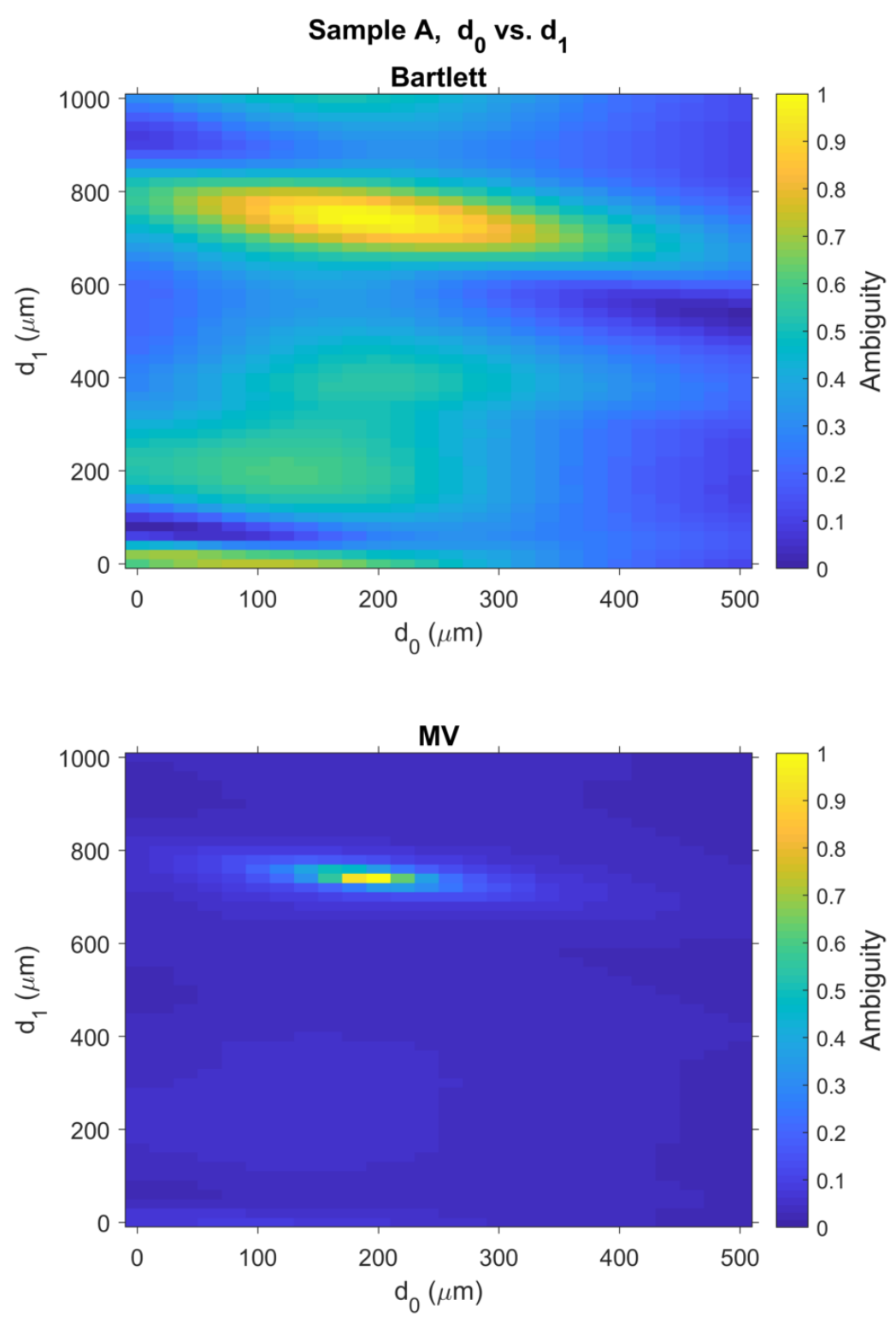

Figure 4.2.5: Matched field ambiguity surfaces provide estimates for the thickness of the calibration layer, $d_{0}$, and polycarbonate layer, $d_{1}$, as illustrated in Figure 4.2.1 with layer thickness for Sample A given in Table 4.2.1. Top panel: Bartlett processor has a global maximum $d_{0}=190 \mu \mathrm{m}$ and $d_{1}=750 \mu \mathrm{m}$. Bottom panel: MV processor has a global maximum $d_{0}=180 \mu \mathrm{m}$ and $d_{1}=750 \mu \mathrm{m}$. The results for $d_{1}$ are consistent with ground truth measurements with a Vernier caliper. Ground truth data is not available for the calibration layer, but the these results are reasonable, and consistent between both Bartlett and MV processors. See Table 4.2.2 and Figure 4.2.8 for a comparison of measurement errors for layers in all samples. 
processor uses adaptive processing to suppresses ambiguities around best estimate. As a result a very narrow peak is observed in the ambiguity surface surrounding the global maximum, located at coordinates $\hat{d}_{0, M V}=180 \mu m$ and $\hat{d}_{1, M V}=750 \mu m$.

Next, for Sample A, the ambiguity surface for $d_{1}$ vs. $d_{2}$ was created for the Bartlett processor by selecting the ambiguity data from the Bartlett processor output for which the $d_{0}=\hat{d}_{0, B}$ and then the remaining ambiguity data was organized into the $2 D$ image shown in the top panel of Figure 4.2.6. A similar process was used to create the ambiguity surface for the MV processor output shown in the bottom panel of Figure 4.2.6. The ambiguity surface for the Bartlett processor shows a relatively broad peak surrounding the global maximum located at coordinates $\hat{d}_{1, M V}=750 \mu m$ and $\hat{d}_{2, B}=70 \mu \mathrm{m}$. As noted above, the MV processor uses adaptive processing to reduce ambiguities around the global maximum, which was also located at coordinates $\hat{d}_{1, M V}=750 \mu \mathrm{m}$ and $\hat{d}_{2, M V}=70 \mu \mathrm{m}$.

\subsubsection{THz MFP Results for Sample D}

Similar to the analysis for Sample A in Section 4.2.5, above, the covariance matrix in Figure 4.2.4 was compared with the replica weight vectors using the Bartlett processor and the MV processors.

For Sample D, the best estimate of the parameter vector from the Bartlett processor was $\hat{\boldsymbol{a}}_{B}=[180,250,60]^{T} \mu \mathrm{m}$, and the best estimate of the parameter vector from the MV processor was $\hat{\boldsymbol{a}}_{\boldsymbol{M V}}=[190,240,60]^{T} \mu \mathrm{m}$. Thus, the estimated thickness for each of the layers were within only $10 \mu m$ of the thicknesses measured for these layers with a digital Vernier caliper. A detailed error analysis for all of the Samples $(A-D)$ is 

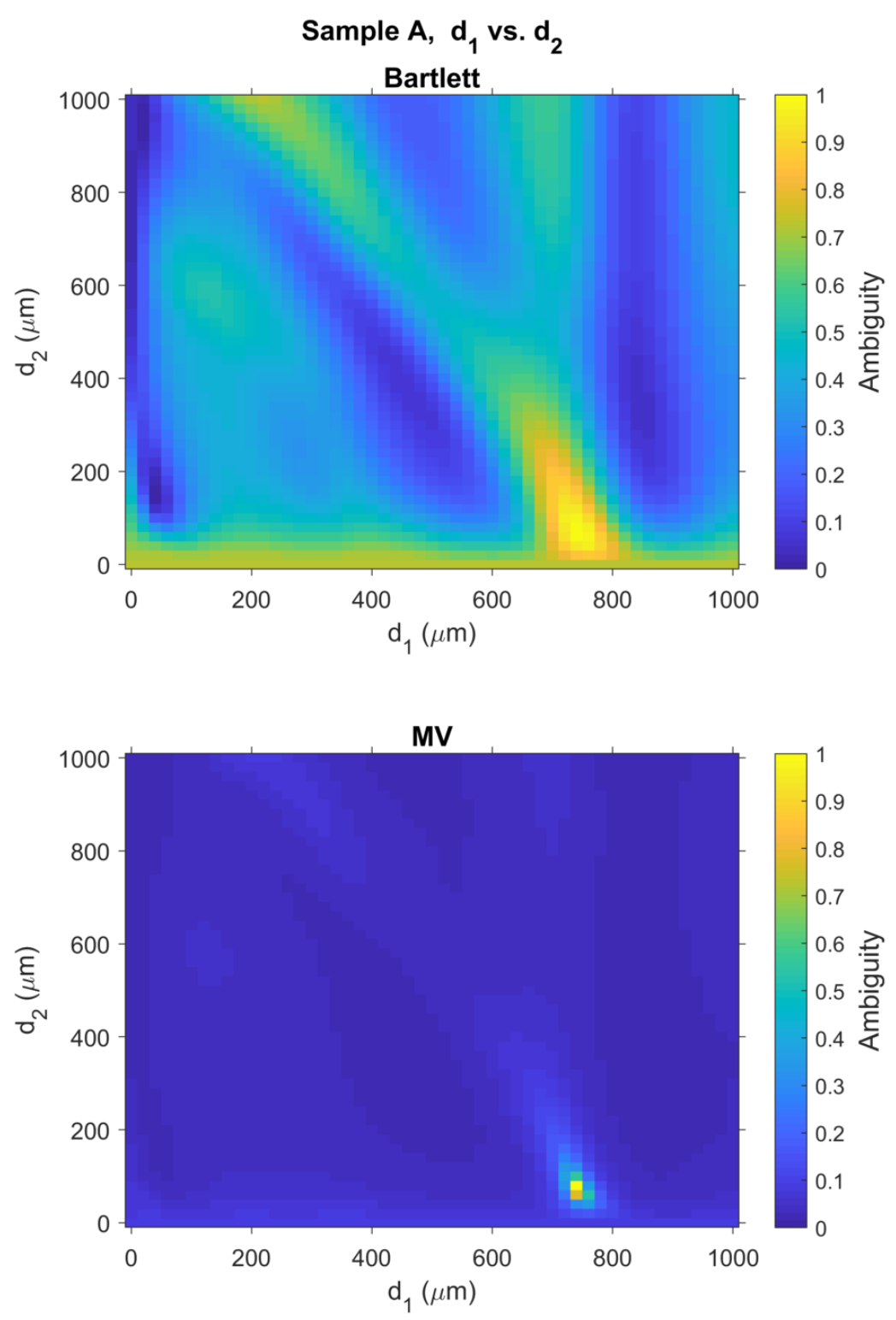

Figure 4.2.6: Matched field ambiguity surfaces provide estimates for the thickness of the polycarbonate layer, $d_{1}$ and the air gap, $d_{2}$, as illustrated in Figure 4.2 .1 with layer thickness for Sample A given in Table 4.2.1. Top panel: Bartlett processor has a global maximum $d_{1}=750 \mu \mathrm{m}$ and $d_{2}=70 \mu \mathrm{m}$. Bottom panel: MV processor has a global maximum $d_{1}=750 \mu \mathrm{m}$ and $d_{2}=70 \mu \mathrm{m}$. These results are consistent with ground truth measurements with a Vernier caliper. See Table 4.2.2 and Figure 4.2.8 for a comparison of measurement errors for layers in all samples. 
provided at the end of this section.

Finally, ambiguity surfaces for Sample D were generated using techniques identical to those used for the ambiguity surface images presented for sample A, above. The top and bottom panels of Figure 4.2.7 show the ambiguity surfaces for Bartlett and MV processors, respectively, for layers $d_{1}$ and $d_{2}$. The ambiguity surface for the Bartlett processor shows a relatively broad peak surround the global maximum located at coordinates $\hat{d}_{1, M V}=250 \mu \mathrm{m}$ and $\hat{d}_{2, B}=60 \mu \mathrm{m}$ with high ambiguity also spread along the bottom edge of the ambiguity surface, i.e. small values of $d_{2}$. As discussed for Sample A above, the MV processor also shows reduced ambiguities around the global maximum for Sample D, located at coordinates $\hat{d}_{1, M V}=240 \mu m$ and $\hat{d}_{2, M V}=60 \mu m$.

\subsubsection{Error Analysis}

The same measurement techniques and signal processing steps discussed in sections 4.2.5 and 4.2.6 were applied to estimate the thicknesses of each of layers shown in Figure 4.2.1 for each of the samples $(A-D)$ listed in Table 4.2.1, and an error analysis was performed to evaluate the performance of the $\mathrm{THz} \mathrm{MFP}$ approach with a conventional measurement.

Ambiguity surfaces (not shown here) were created for each of the layers in each of the samples. In each case the global maximum of the Bartlett and MV ambiguity surfaces appeared at approximately the same depth coordinates as discussed above, and the MV processor suppressed ambiguities around the global maximum, similar to the ambiguity surface results plotted in Figures 4.2.5 - 4.2.7, above. The best estimate of the thicknesses from the Bartlett and MV processors are recorded in 

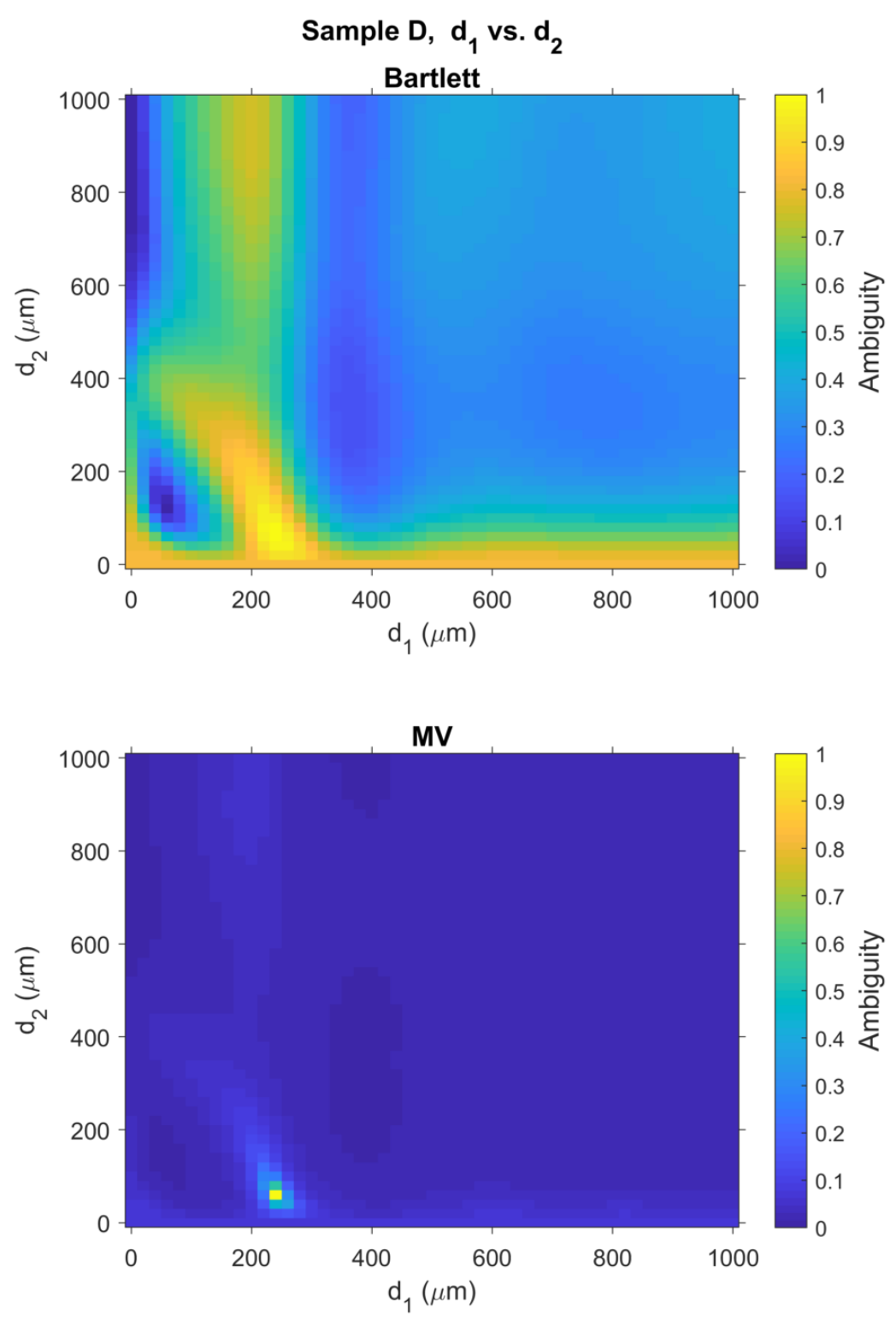

Figure 4.2.7: Matched field ambiguity surfaces provide estimates for the thickness of the polycarbonate layer, $d_{1}$ and the air gap, $d_{2}$, as illustrated in Figure 4.2.1 with layer thickness for Sample D given in Table 4.2.1. Top panel: Bartlett processor has a global maximum $d_{1}=250 \mu \mathrm{m}$ and $d_{2}=60 \mu \mathrm{m}$. Bottom panel: MV processor has a global maximum $d_{1}=240 \mu \mathrm{m}$ and $d_{2}=60 \mu \mathrm{m}$. These results are consistent with ground truth measurements with a Vernier caliper. See Table 4.2.2 and Figure 4.2.8 for a comparison of measurement errors for layers in all samples. 
Table 4.2.2, along with thickness measurements from a digital Vernier caliper. Note, Vernier caliper measurements are not available for comparison with the calibration layer.

The same measurement resolution was used for the Vernier caliper and the $\mathrm{THz} \mathrm{MFP}$ thickness estimates. The measurement resolution and accuracy of the Vernier caliper were $10 \mu m$ and $20 \mu m$, respectively. Similarly, the thickness interval between each of the trial thicknesses that were used to create the replicas was $10 \mu \mathrm{m}$. The results of the thickness estimates from THz MFP are in excellent agreement with the measurements from the Vernier caliper. All of the differences between the THz MFP results and the Vernier caliper measurements are within the measurement resolution of the Vernier caliper.

A visual comparison of the thickness data listed is Table 4.2.2 is provided in Figure 4.2.8. For each of the Vernier caliper measurements listed in the table, the corresponding thickness estimates from $\mathrm{THz}$ MFP with the Bartlett and MV processors are plotted as blue circles and red squares, respectively. The dashed line represents an ideal case of equality of the Vernier caliper measurement and the THz MFP thickness estimate. Thus, all of the measurement data from the THz MFP approach is in close agreement with the Vernier caliper data.

\subsection{THz NDE for Thin Films of Acrylic Paint}

This section describes $\mathrm{THz}$ experiments that use the MFP approach for NDE of an acrylic paint on a metal substrate. Thus, the examples considered here could be representative of industrial NDE applications which require NDE of paints or other 


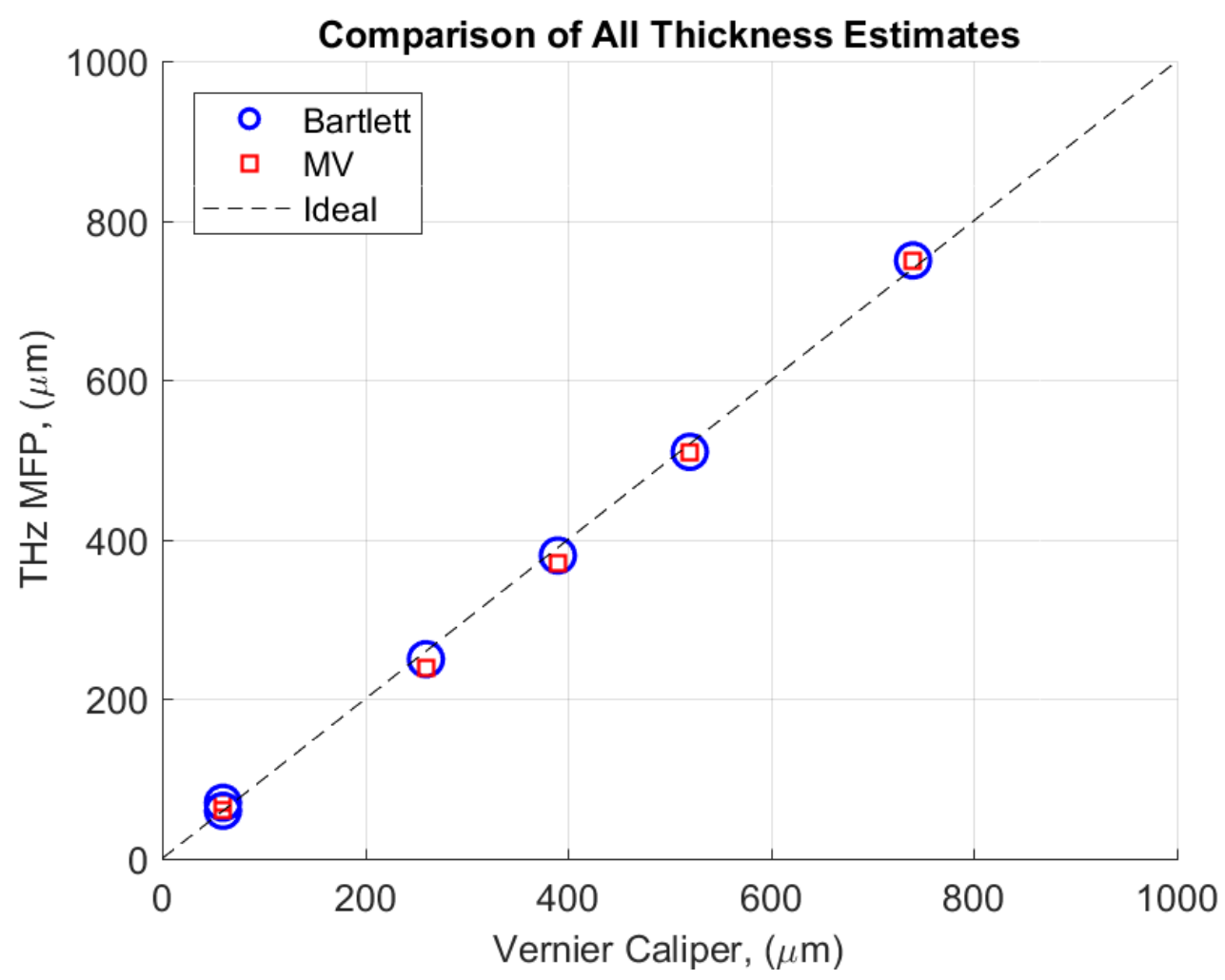

Figure 4.2.8: For each of the Vernier caliper measurements listed in Table 4.2.2, the corresponding thickness estimates from $\mathrm{THz}$ MFP with the Bartlett and MV processors are plotted as blue circles and red squares, respectively. The dashed line represents an ideal case of equality of the Vernier caliper measurement and the $\mathrm{THz}$ MFP thickness estimate. Thus, all of the measurement data from the THz MFP approach is in close agreement with the Vernier caliper data. 
Table 4.2.2: Thickness estimates obtained from THz MFP with the Bartlett and MV objective functions for the experiment configuration shown in Figure 4.2.1. All of the differences between the $\mathrm{THz}$ MFP results and the Vernier caliper measurements are within the measurement resolution of the Vernier caliper $(20 \mu \mathrm{m})$.

\begin{tabular}{|c||c|c||c|c||}
\hline $\begin{array}{c}\text { Sample } \\
\text { ID }\end{array}$ & $\begin{array}{c}\text { Layer } \\
\text { ID }\end{array}$ & $\begin{array}{c}\text { Vernier Cal. } \\
(\mu m)\end{array}$ & $\begin{array}{c}\text { THz MFP Bartlett } \\
(\mu m)\end{array}$ & $\begin{array}{c}\text { THz MFP: MV } \\
(\mu m)\end{array}$ \\
\hline \hline A & $d_{0}$ & NA & 190 & 180 \\
\hline A & $d_{1}$ & 740 & 750 & 750 \\
\hline A & $d_{2}$ & 60 & 70 & 70 \\
\hline \hline B & $d_{0}$ & NA & 160 & 160 \\
\hline B & $d_{1}$ & 520 & 510 & 510 \\
\hline B & $d_{2}$ & 60 & 70 & 160 \\
\hline \hline C & $d_{0}$ & NA & 160 & 370 \\
\hline C & $d_{1}$ & 390 & 380 & 70 \\
\hline C & $d_{2}$ & 60 & 60 & 190 \\
\hline \hline D & $d_{0}$ & NA & 180 & 60 \\
\hline D & $d_{1}$ & 260 & 250 & 60 \\
\hline D & $d_{2}$ & 60 & & \\
\hline
\end{tabular}

surface coating materials.

In this study, the material properties (frequency-dependent index of refraction and extinction coefficient) of the paint are available from a separate transmission measurement, and the objective is to estimate the thickness of the paint layer. Several possible thicknesses for the paint layer are considered here.

\subsubsection{Terahertz Measurement System}

The experiments discussed here were performed the NEAR-Lab at PSU, with the T-Ray 4000 as discussed in section 4.2.1. The THz TDS system was configured for 
monostatic measurements at normal incidence as illustrated in Figure 4.3.1.

As discussed in Section 3.3.3, THz MFP in reflection configuration requires the $\mathrm{THz}$ source signal to be approximated with a reference measurement from a mirror. The left side of Figure 4.3.1 shows the configuration for the $\mathrm{THz}$ reference measurement from a gold mirror. A total of 10,000 reference waveforms were averaged to maximize the SNR. After performing a DFT, the reference spectrum could be used to approximate the source spectrum, $D(f)$, needed to generate the replica spectra, $R_{r}(f, \boldsymbol{a})$, in Eq. 3.3.7.

\subsubsection{Paint Film Samples}

The right side of Figure 4.3.1 illustrates the measurement configuration for a singlelayer paint film on an aluminum substrate. As discussed in the previous section, there is always a small offset distance between the surface of the sample under test and the surface of the mirror during the reference measurement. This calibration distance $\left(d_{0}\right.$ in Figure 4.3.1) is accounted for with MFP by including an additional thin layer of air above the sample's surface when generating replica spectra with the propagation model [30].

The paint was a premium automotive spray paint purchased from an automotive parts store. Dupli-Color@ Perfect Matchß paint is an acrylic lacquer aerosol paint that is formulated to match the exact color of the coating that was applied at the original factory [61]. A bright red acrylic paint (manf. part number 81 WA8774) that was designed to duplicate original General Motors (GM) colors was chosen. Figure 4.3.2 shows a photo the spray paint. 


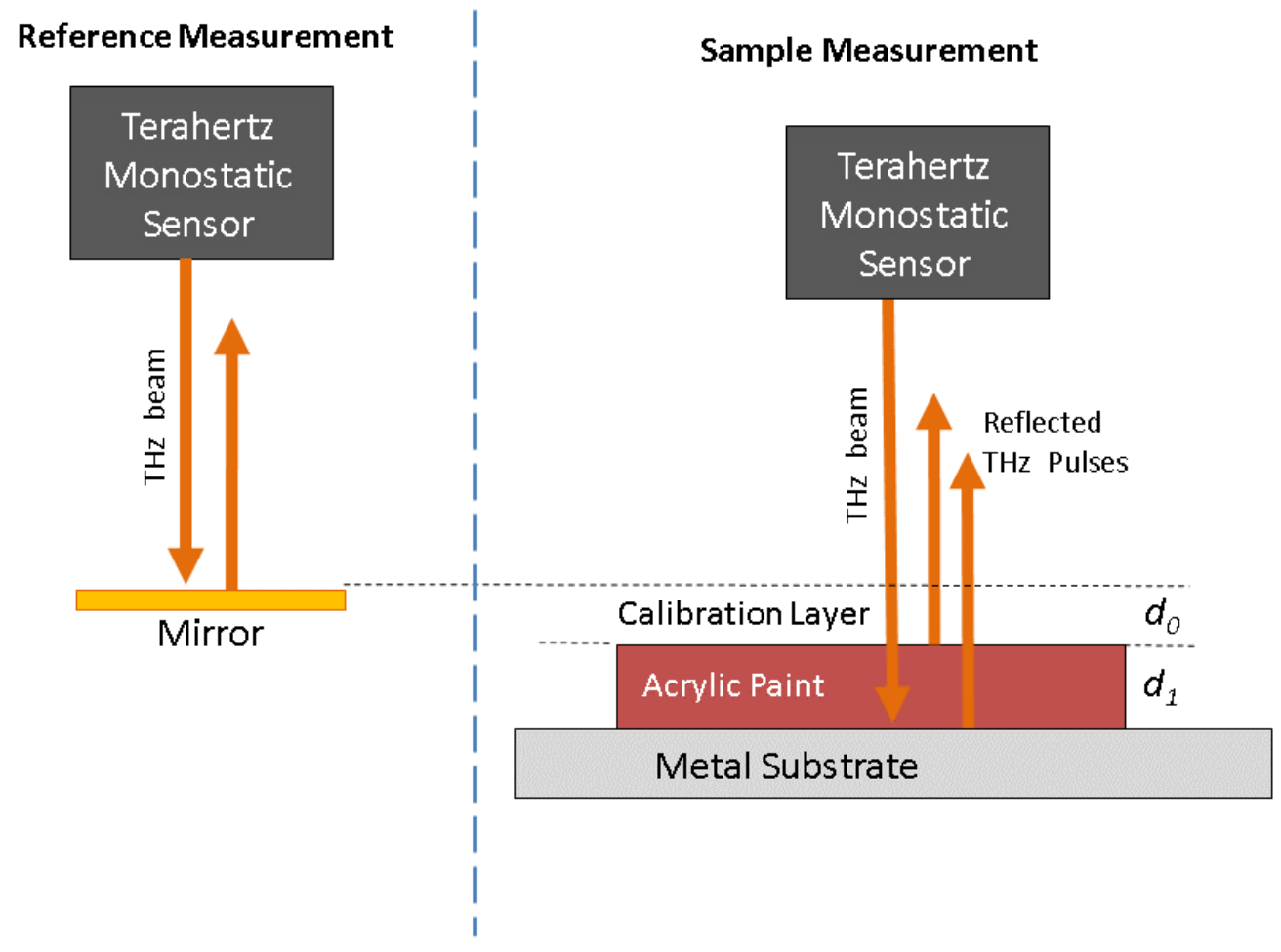

Figure 4.3.1: Illustration of the $\mathrm{THz} \mathrm{NDE}$ configuration for paint film experiments (not to scale). Left: Measurement configuration for the THz reference, which is used to approximate the $\mathrm{THz}$ source signal in MFP processing. Right: Measurement configuration for the layered sample under test. A calibration layer (air) with unknown thickness, $d_{0}$, accounts for the offset distance between the reference mirror and the surface of the sample. Here, the paint layer thickness is denoted as $d_{1}$. THz MFP is used to estimate the thicknesses of both layers $\left(d_{0}\right.$ and $\left.d_{1}\right)$, simultaneously. 


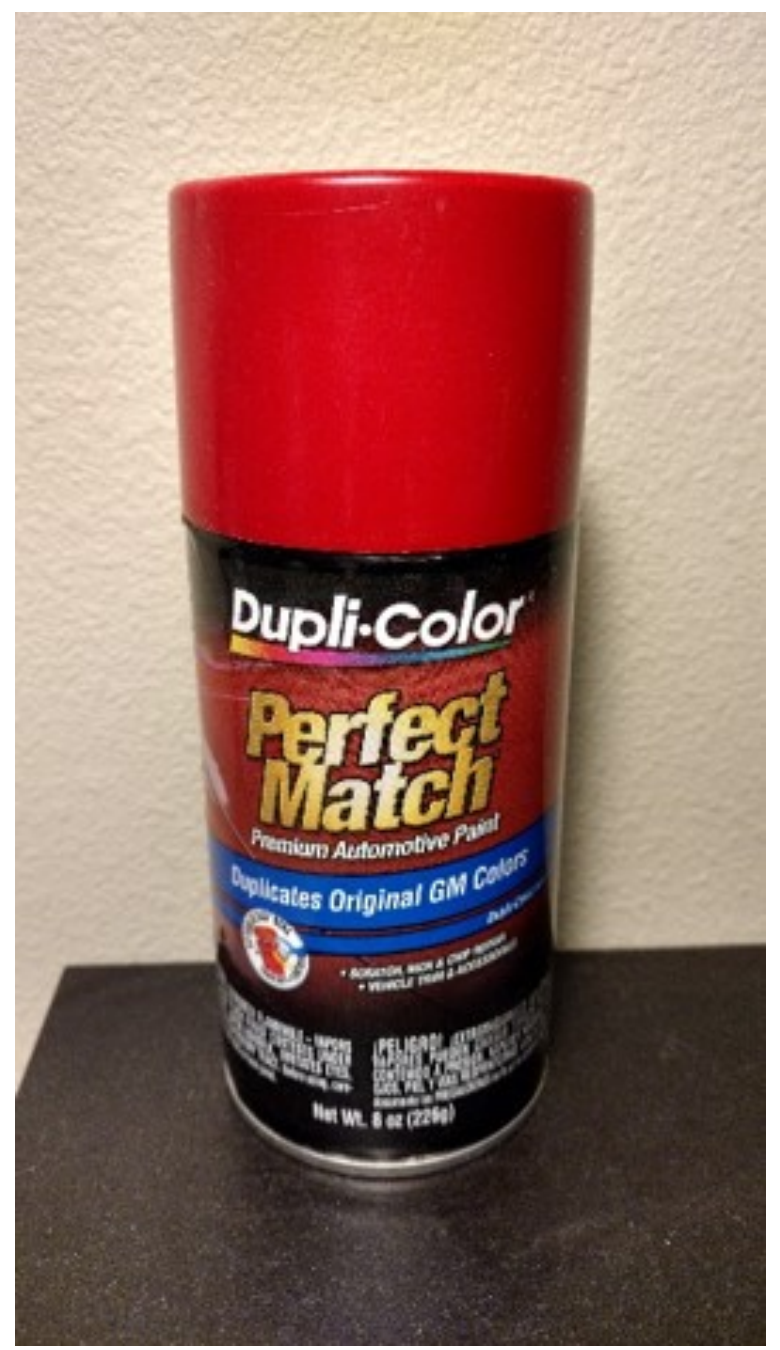

Figure 4.3.2: Dupli-Color@ Perfect Match@ paint is an acrylic lacquer aerosol paint designed to duplicate original General Motors (GM) colors [61]. 


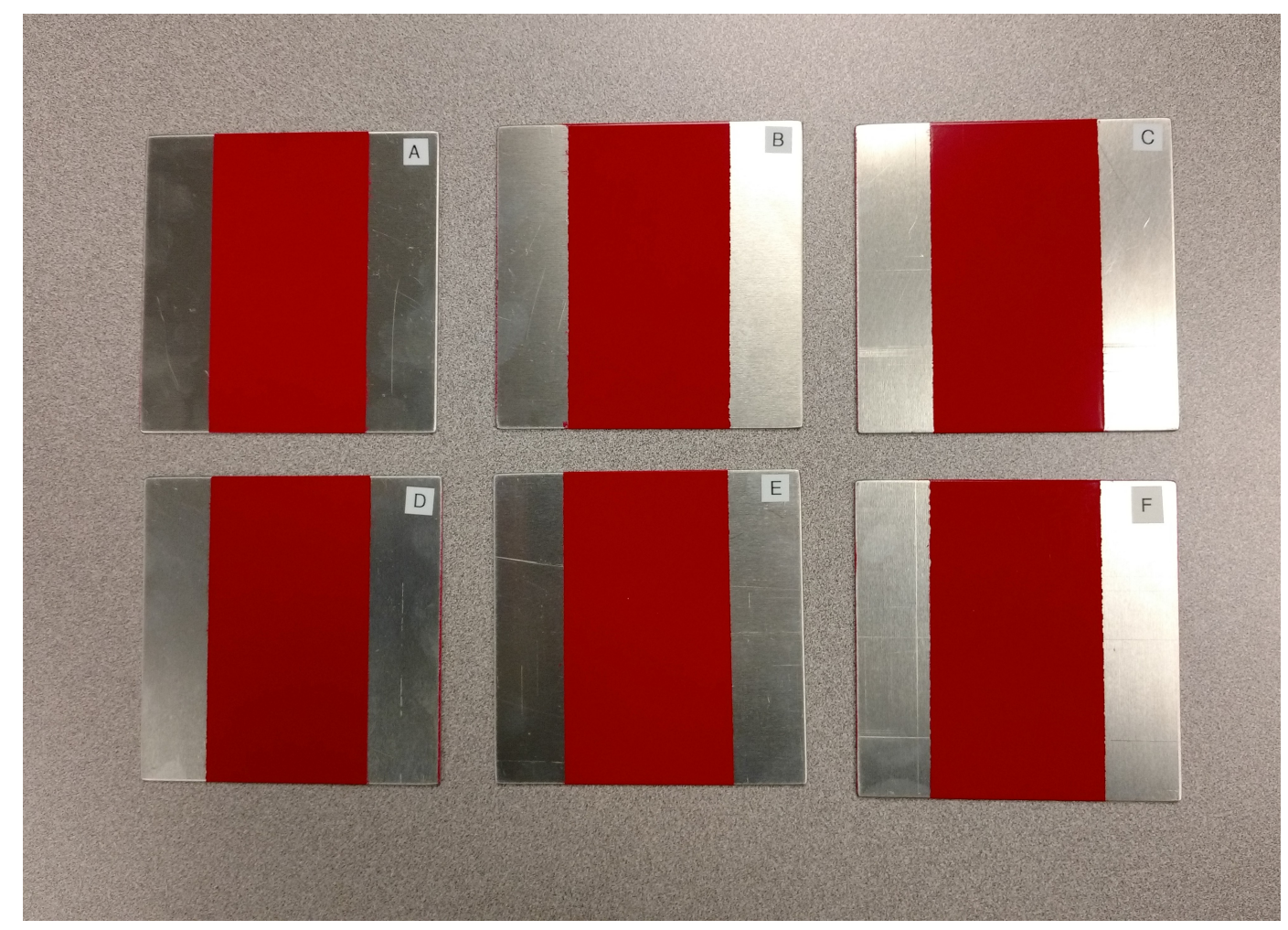

Figure 4.3.3: Six (6) different paint samples (A - F) were created by spray painting aluminum plates with Dupli-Color $\AA$ Perfect Match $\AA$ paint. Sample A was created by applying several coats of spay paint to make the sample with the thickest layer. Fewer coats were applied to the subsequent sample plates (B - F).

In this experiment, the thickness of the paint layer is denoted $d_{1}$, as shown in Figure 4.3.1. Six (6) different paint samples (A - F) were created by spray painting aluminum plates that were 4 inches $\times 4$ inches $\times 1 / 8$ inch thick. Figure 4.3 .3 shows a photo of the samples. Sample A was created by applying several coats of spay paint to make the sample with the thickest layer. Fewer coats were applied to the subsequent sample plates $(\mathrm{B}-\mathrm{F})$, so that the thinnest coat was applied to Sample F.

The thickness of the paint film on each sample plate was measured with a digital coating thickness tester manufactured by CEM [62]. The CEM DT-156 shown in Figure 4.3.4 uses eddy currents induced in the aluminum substrate to measure coating 
thicknesses. Therefore, the electromagnetic sensor on the bottom of the instrument must make direct contact with paint to make a measurement. The instrument is calibrated to account for dielectric coatings between the instrument's sensor and the aluminum substrate, and the digital output reports coating thickness measurements with a resolution of $0.1 \mu \mathrm{m}$. The output can also be saved to a file for statistical analysis on a PC. The manufacturer specifies the total measurement uncertainty as

$$
U= \pm(s+(1 \%) \bar{x}+1 \mu m)
$$

where $\bar{x}$ is the mean and $s$ is the standard deviation.

The mean and standard deviation for 30 measurements near the center of each plate is recorded in Table 4.3.1. This measurement data is used to evaluate the accuracy of the thickness estimates from $\mathrm{THz}$ MFP presented later in this section.

Table 4.3.1: Six (6) samples (A - F) of Dupli-Color@ Perfect Match® paint film on aluminum substrate were measured with the CEM DT-156 coating thickness tester [62] shown in Figure 4.3.4. Thirty (30) measurements for each sample were used to compute the statistics shown. This data is used to evaluate the accuracy of the $\mathrm{THz}$ MFP results presented later in this section.

\begin{tabular}{|c|c|c|c|c|c|}
\hline Sample & $\begin{array}{c}\text { Minimum } \\
\text { Thickness } \\
\mu m\end{array}$ & $\begin{array}{c}\text { Maximum } \\
\text { Thickness } \\
\mu m\end{array}$ & $\begin{array}{c}\text { Mean } \\
\text { Thickness } \\
\mu m\end{array}$ & $\begin{array}{c}\text { Standard } \\
\text { Deviation } \\
\mu m\end{array}$ & $\begin{array}{c}\text { Total } \\
\text { Uncertainty } \\
\mu m\end{array}$ \\
\hline A & 107 & 113 & 110 & \pm 1.8 & \pm 3.9 \\
\hline B & 68.0 & 73.0 & 72.0 & \pm 1.3 & \pm 3.0 \\
\hline C & 47.6 & 53.0 & 49.7 & \pm 1.2 & \pm 2.7 \\
\hline D & 26.1 & 32.7 & 28.4 & \pm 1.9 & \pm 3.2 \\
\hline E & 30.1 & 37.0 & 34.1 & \pm 1.8 & \pm 3.1 \\
\hline F & 13.9 & 17.2 & 16.2 & \pm 1.0 & \pm 2.1 \\
\hline
\end{tabular}




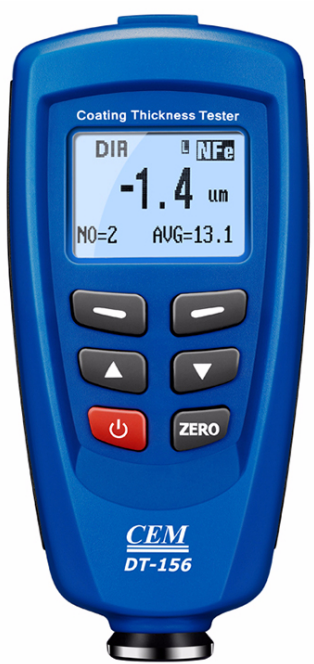

Figure 4.3.4: Photo of the CEM DT-156 coating thickness tester, which uses eddy currents induced in the aluminum substrate to measure coating thicknesses [62]. The electromagnetic sensor on the bottom of the instrument must make direct contact with paint to make a measurement. The instrument is calibrated to account for dielectric coatings between the instrument's sensor and the aluminum substrate and reports coating thickness measurements in microns.

For the THz NDE example discussed in this dissertation, the objective is to estimate the thickness of layers within a known medium, a propagation model is used to generate replica spectra for various possible layer thicknesses, and MFP is used to compare the replicas with measurements. The propagation modeling required to make replicas for the $\mathrm{THz}$ NDE experiment requires a priori knowledge of the index of refraction for the sample materials.

$\mathrm{THz}$ measurements were performed in transmission configuration with a layer of the Dupli-Color@ Perfect Match@ paint film on a silicon wafer substrate to extract the material properties of paint film. Figure 4.3.5 shows a photo of the wafer with paint coating. The uncoated half of the silicon wafer was used as the reference measurement for the $\mathrm{THz}$ spectroscopy algorithm. The resulting complex index of refraction and absorption coefficient for the paint are shown in Figure 4.3.6. 


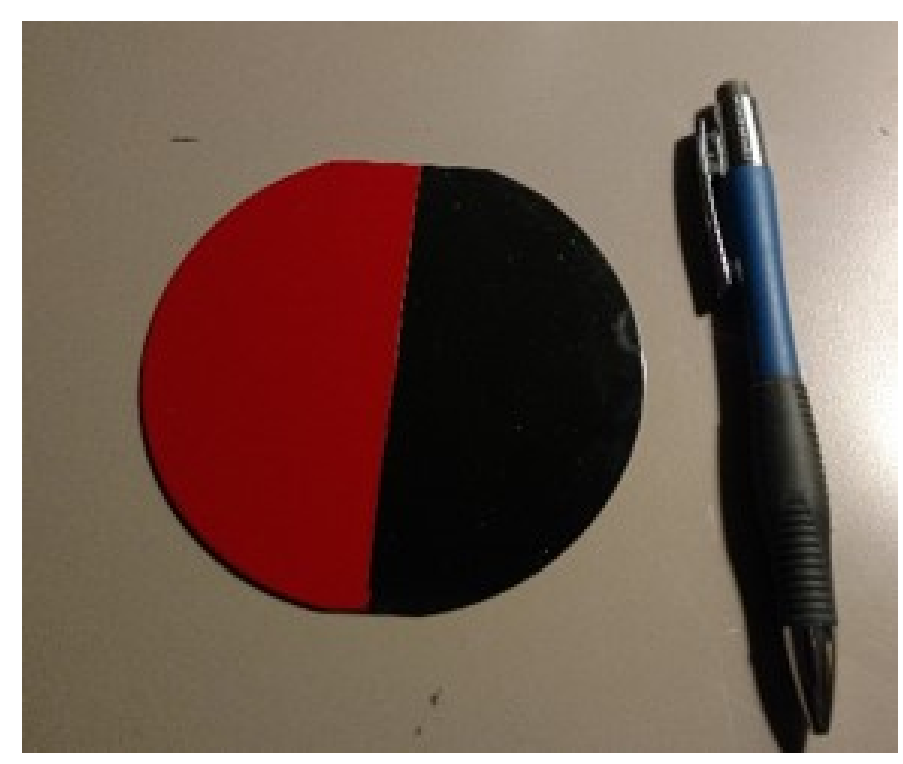

Figure 4.3.5: Part of a silicon wafer was coated with a layer of Dupli-Color $囚$ Perfect Match $\AA$ paint. This sample was used in a separate $\mathrm{THz}$ spectroscopy experiment to extract the index of refraction, which is needed as an input when generating replicas to be used for $\mathrm{THz} \mathrm{MFP}$.

\subsubsection{Measurement Data Processing}

The monostatic THz sensor configuration shown in Figure 4.3.1 was used to record THz waveforms above sample A from Table 4.3.1. A total of 300 waveforms were each recorded at a single position with minimal measurement integration time (10 ms/waveform). The waveforms were truncated within a time window with duration of 60 ps surrounding the reflected pulses from the surface and the air gap below the surface.

The top panel of Figure 4.3.7 shows the mean THz waveform for Sample A. The middle plot of Figure 4.3.7 shows the spectrum of the mean waveform in the top panel, truncated with the bandwidth of $0.1-1.3 \mathrm{THz}$. Note, that no multipath are observed in the $\mathrm{THz}$ waveform or spectrum because the pulses reflected from the 

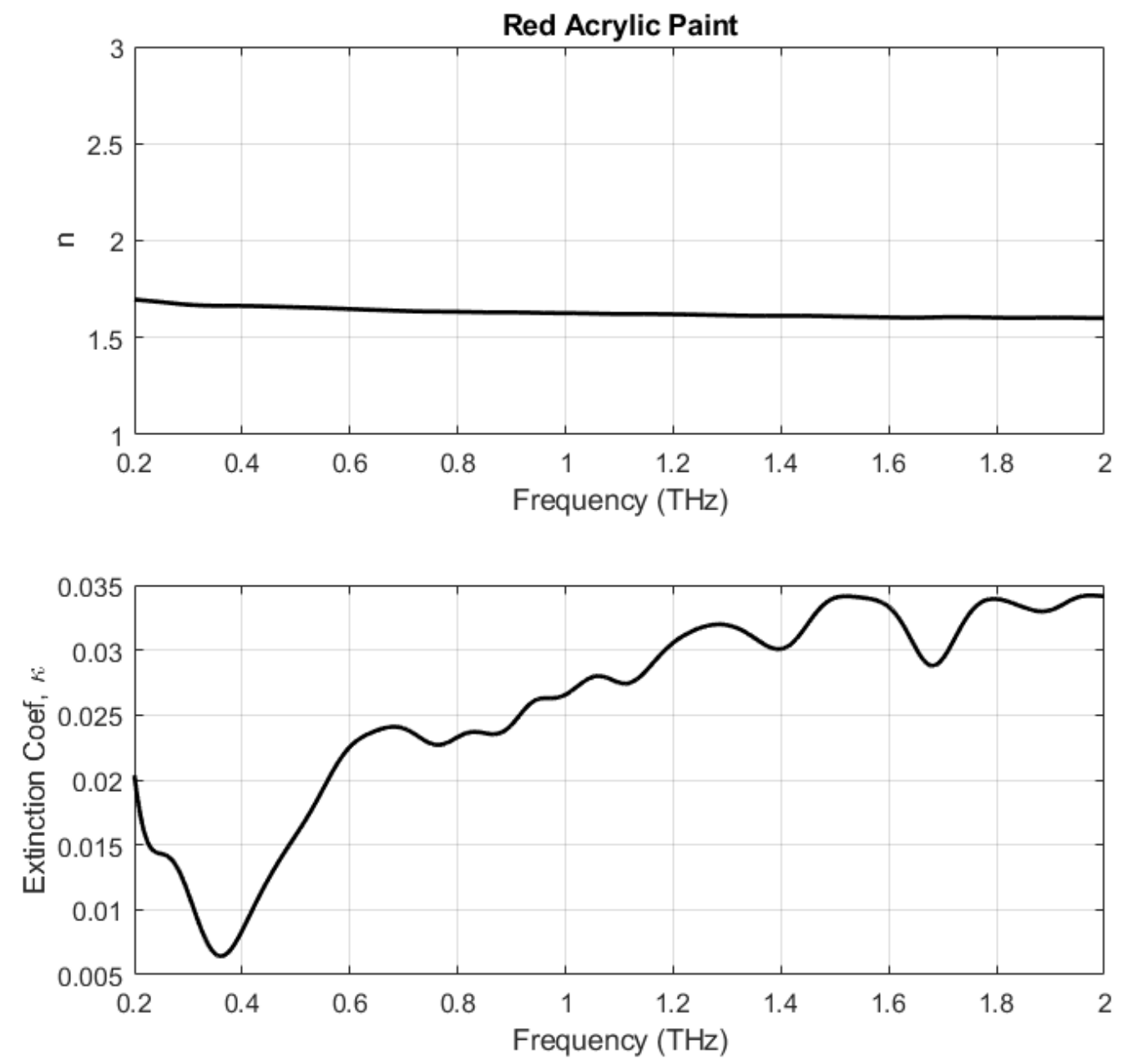

Figure 4.3.6: Complex index of refraction for the red acrylic paint were calculated from $\mathrm{THz}$ transmission measurements with the silicon wafer sample shown in Figure 4.3.5. Top panel: real part of the refractive index, $n$. Bottom Panel: Extinction coefficient, $\kappa$. 
upper and lower surfaces of the paint film overlap on one another.

An FFT was performed on the each of the truncated $\mathrm{THz}$ waveforms and the 300 resulting spectra were truncated within the bandwidth of $0.1-1.3 \mathrm{THz}$ for maximum SNR. Each of the spectra were normalized to unit vectors as discussed in Sections 3.3.4 and 3.3.5. This normalization is not necessary for MFP, but provides a maximum possible output of unity in the Bartlett processor if the replica weight vectors are also normalized to unit vectors [45], [49]. The covariance was then estimated using Eq. 3.3.9 with $L=145$ frequency bins and $Z=300$ snapshots. The resulting covariance matrix is a $L \times L$ square matrix of complex values. The bottom panel of Figure 4.2.3 shows the absolute value of the covariance matrix on a dB scale.

Data was processed for the other Samples $(B-F)$ in a manner similar to the method discussed for Sample A above. Each of the covariance matrix plots are similar to the one shown in Figure 4.3.7, and therefore are not shown here.

\subsubsection{Generating Replica Spectra}

As discussed in Section 3.3, the MFP approach compares the covariance matrix with a set of modeled replica fields. The transfer matrix model discussed in Appendix A was used to generate a set of transfer functions, $H_{s}(f, \boldsymbol{a})$, for the layered media sample illustrated in Figure 4.3.1, where the parameter vector, $\boldsymbol{a}$, contained the thicknesses of the two layers, i.e. $\boldsymbol{a}=\left[d_{0}, d_{1}\right]^{T}$.

A range of possible thicknesses were considered for each of the two layers. A total of 351 thicknesses from $0-700 \mu m$ in $2 \mu m$ intervals were considered for the calibration layer, $d_{0}$, and a total of 101 thicknesses from $0-200 \mu m$ in $2 \mu m$ intervals were con- 

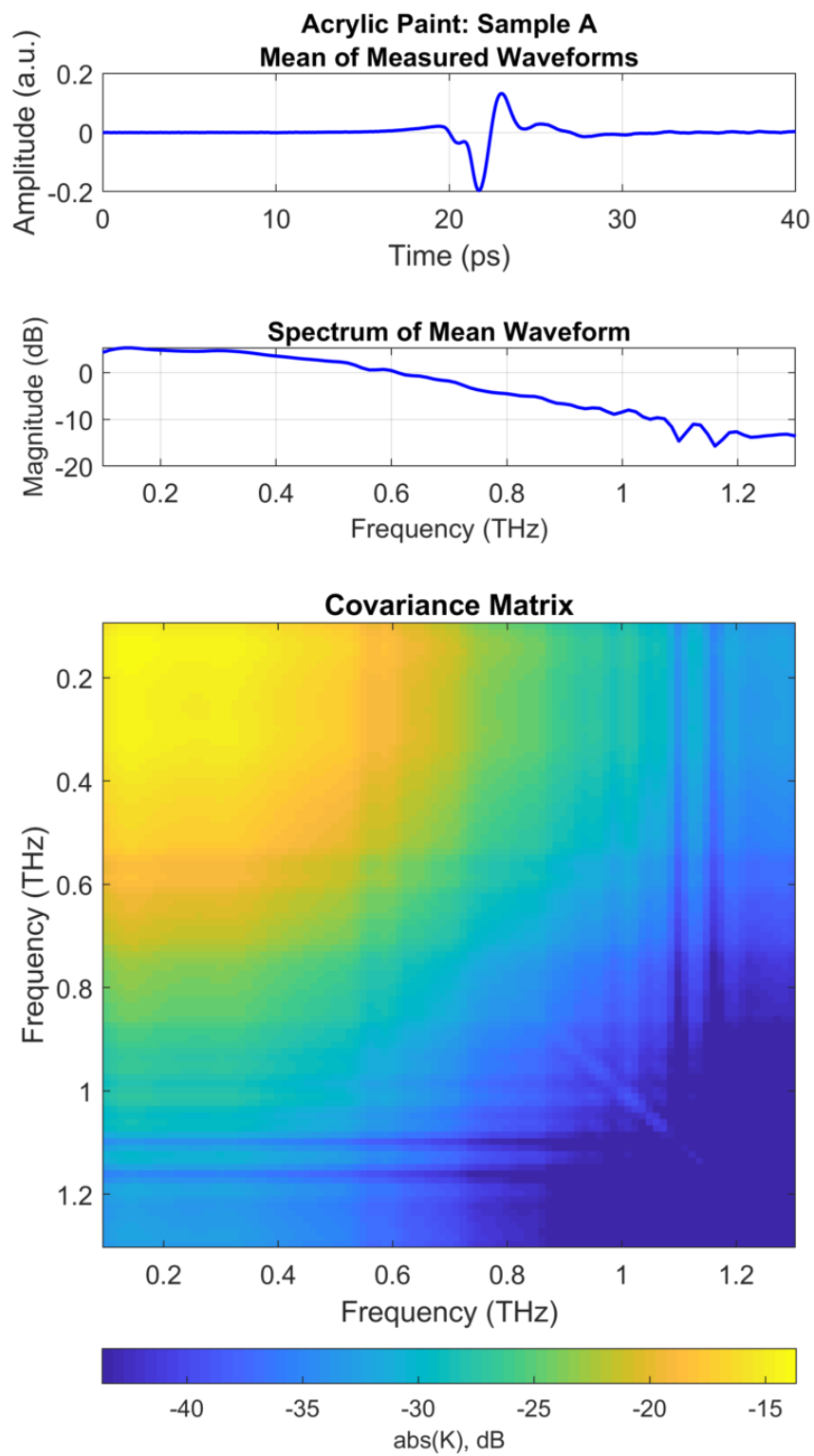

Figure 4.3.7: Sample A Measurement data Top Panel: Mean of measured THz waveforms. Middle Panel: Spectrum of mean waveform in the top panel. Bottom Panel: Covariance matrix computed with Eq. 3.3.9 using the spectrum of each of the 300 measured waveforms. 
sidered for the acrylic paint layer, $d_{1}$. Thus, there were a total of $351 \times 101=35,451$ replica spectra created for this experiment.

The index of refraction (Eq. A.2.3) used for the calibration layer was $n=1$ for all frequencies. Thus, the absorption spectra of the air was neglected because it is expected to have negligible impact on the measured spectra for these thin layers with thicknesses on the order of a few hundred microns. The frequency-dependent index of refraction for the acrylic layer was taken from a separate transmission measurement as discussed in Section 4.3.2 and shown in Figure 4.3.6.

As noted in Section 4.2.4, the propagation model in Appendix A is a frequency-domain model that computes the transfer function for the layered stack, $H_{s}(f, \boldsymbol{a})$, at finite frequency bins, and Fourier synthesis is required to estimate the impulse response for the sample layers. This is because the model assumes an infinite number of multipath reflections within the sample layers whereas the $\mathrm{THz}$ measurement system truncates mutlitpath reflections outside a measurement window of finite duration (320 ps for the T-Ray 4000 used in this study). Therefore, to avoid aliasing of the multipath arrivals in the time-domain, a frequency resolution (four times smaller than the resolution of the measurement data) was used to generate the simulated spectra, $H_{s}(f, \boldsymbol{a})$.

Several signal processing steps were required to compute replicas of the received spectra. The transfer functions were generated for positive frequencies using the propagation model and then zero-padded to create the analytic spectrum. An inverse FFT was then performed to get the analytic signal in time domain, which was converted to a real signal (impulse response). This real signal was then interpolated onto the same time axis as the truncated measured waveforms in order to eliminate multipath arrivals in the model that would be outside the measurement time win- 
dow of the THz-TDS system. An FFT was then performed on the resulting time domain impulse response (similar to the FFT performed on the measured data), and the resulting spectrum was multiplied by the $\mathrm{THz}$ source spectrum $D(f)$ for each transfer function, $H_{s}(f, \boldsymbol{a})$, as discussed in Section 3.3.4, to give the replica spectra for the received $\mathrm{THz}$ spectra, $R_{\text {sim }}(f, \boldsymbol{a})$. Finally, the normalized weight vectors were computed using Eq. 3.3.8.

Due to the similarity of the samples $(A-D)$, the process of computing the replica spectra only needed to be computed once. The resulting set of replicas were stored in a file and loaded for comparison with each of the covariance matrix for each sample $A-F$ in this study.

\subsubsection{THz MFP Results for Paint Film Samples}

Similar to the analysis discussed in Section 4.2.5, the covariance matrix in Figure 4.3.7 was compared with the replica weight vectors using the Bartlett processor and the MV processor using Equations 3.3.11 and 3.3.12, respectively. The output, $P(\boldsymbol{a})$ of each processor is a scalar value for each possible parameter vector, $\boldsymbol{a}$. Thus, parameter vector corresponding to the maximum value of the processor output is taken to be the the best estimate, $\hat{\boldsymbol{a}}$, for the unknown parameters.

For Sample A, the best estimate of the parameter vector from the Bartlett processor was $\hat{\boldsymbol{a}}_{\boldsymbol{B}}=[198,112]^{T} \mu \mathrm{m}$, and the best estimate of the parameter vector from the MV

processor was $\hat{\boldsymbol{a}}_{\boldsymbol{M V}}=[196,112]^{T} \mu \mathrm{m}$. Thus, the estimated thicknesses were nearly the same for both processors. The exact thickness of the calibration layer is unknown, but the results obtained here are reasonable for this experiment configuration. The 
estimated thickness from both the Bartlett and MV processors was $112 \mu m$, which is in excellent agreement with the thickness that was measured with the CEM DT-156 Coating Thickness Tester, which showed a thickness of $110 \pm 3.9 \mu m$ for the Sample A paint layer (See Table 4.3.1). Note, an error analysis for all the paint film samples $(\mathrm{A}-\mathrm{F})$ is shown in Section 4.3.6.

As discussed in Section 3.3.7, ambiguity surfaces are often used in the MFP literature to evaluate the ambiguity around the estimated parameters. Similar to the results shown in Sections 4.2.5 and 4.2.6, a $2 D$ image of the ambiguity surface for the Bartlett processor output for Sample A was generated for $d_{0}$ vs. $d_{1}$. The $2 D$ ambiguity data for paint Sample A is shown in the top panel of Figure Figure 4.3.8.

Thus, each pixel in the image gives the ambiguity of the Bartlett processor corresponding to the replica spectrum that was computed with the values of $d_{0}$ and $d_{1}$ given by the horizontal and vertical axes of the image, respectively. The ambiguity surface for the Bartlett processor in the top panel of Figure 4.3.8 shows relatively high ambiguity surrounding the global maximum, located at coordinates $\hat{d}_{0, B}=198 \mu \mathrm{m}$ and $\hat{d}_{1, B}=112 \mu m$.

Similarly, for Sample A, a $2 D$ image of the ambiguity surface for the MV processor output was generated for $d_{0}$ vs. $d_{1}$, as shown in the bottom panel of Figure 4.3.8. As noted in Section 3.3.6.2, the MV processor uses adaptive processing to suppresses ambiguities around best estimate. As a result a very narrow peak is observed in the ambiguity surface surrounding the global maximum, located at coordinates $\hat{d}_{0, M V}=$ $196 \mu m$ and $\hat{d}_{1, M V}=112 \mu m$.

Similar to the analysis for paint Sample A, above, the covariance matrices for paint 
samples B - F were compared with the replica weight vectors using the Bartlett processor and the MV processors. The ambiguity surfaces for samples B - F are shown in Figures 4.3.8 through 4.3.13. The best estimate from each processor (global maximum in the surface images) is listed in the caption of each figure.

\subsubsection{Error Analysis}

The best estimate of the thicknesses from the Bartlett and MV processors for each of the 6 paint samples $(\mathrm{A}-\mathrm{F}$ ) are recorded in Table 4.3.2, along with mean thickness measurements from a digital CET DT-156 Coating Thickness Tester. Note, measurements are not available for the calibration layer.

The data in Table 4.3.2 indicates that the estimated paint layer thickness $d_{1}$ for both the Bartlett and MV processors was in excellent agreement with the thickness that was measured with the CEM DT-156 Coating Thickness Tester for samples A - E. The estimated thickness for paint sample F differed slightly from the CEM DT-156 measurements. Sample F was the thinnest sample in this study with a thickness of only $16.2 \pm 2.1 \mu \mathrm{m}$. It is possible that this small thickness may be just beyond the capability of the THz MFP method. Analysis of the measurement resolution limitations remains as a future work, as discussed in Section 5.1.

It is interesting to note that the estimates for the calibration layer thickness (layer $d_{0}$ ) are thicker for the samples that have a thinner paint layer (layer $d_{1}$ ). For example, the Bartlett processor estimates a calibration layer thickness for sample B that is $248-198=50 \mu m$ thicker than the calibration layer for sample A. This is consistent with expectations because the paint layer for sample B is approximately $110-72=$ 

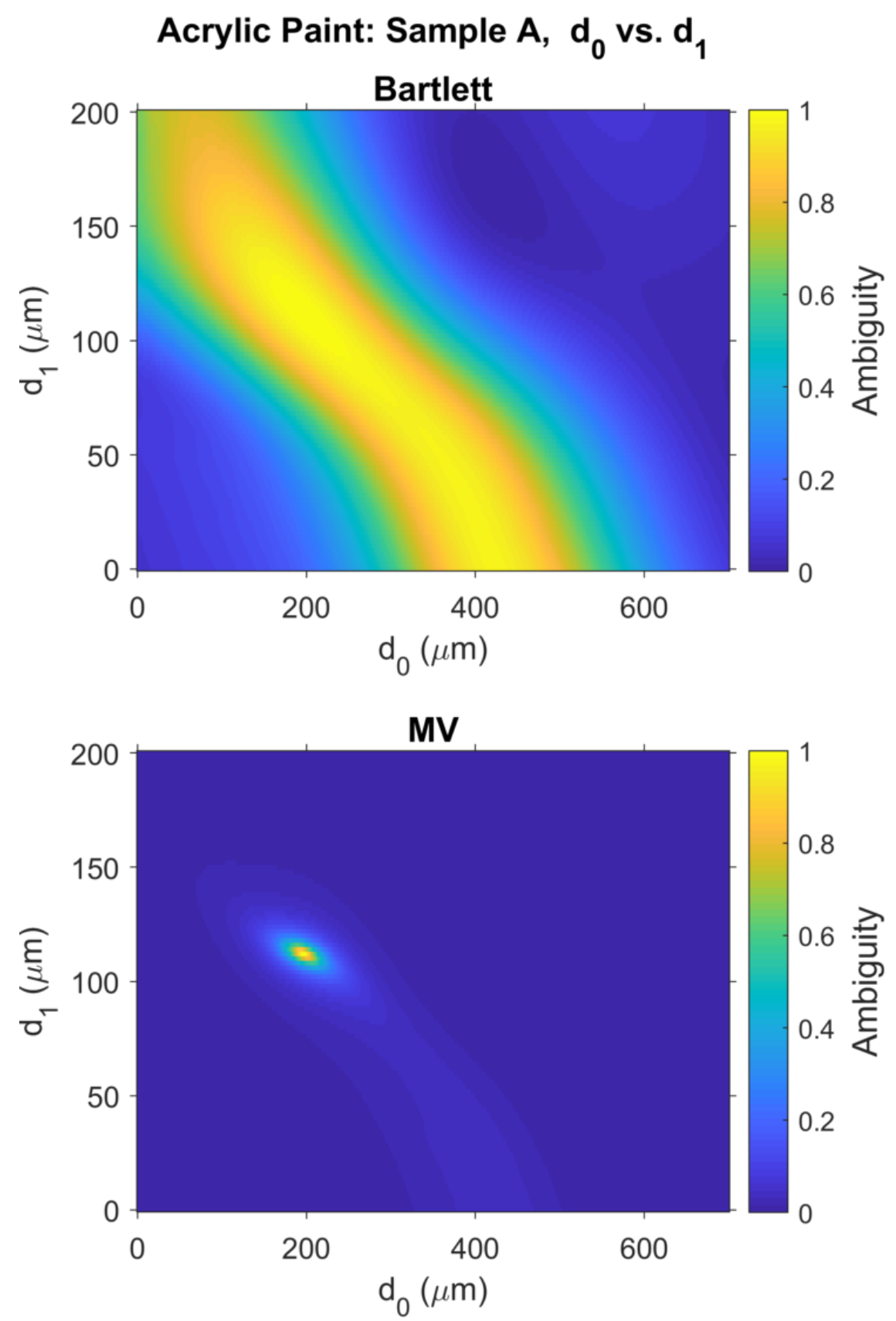

Figure 4.3.8: Sample A: Matched field ambiguity surfaces provide estimates for the thickness of the calibration layer, $d_{0}$, and acrylic paint layer, $d_{1}$.

Top panel: Bartlett processor global maximum at $d_{0}=198 \mu \mathrm{m}$ and $d_{1}=112 \mu \mathrm{m}$. Bottom panel: MV processor global maximum at $d_{0}=196 \mu \mathrm{m}$ and $d_{1}=112 \mu \mathrm{m}$. 

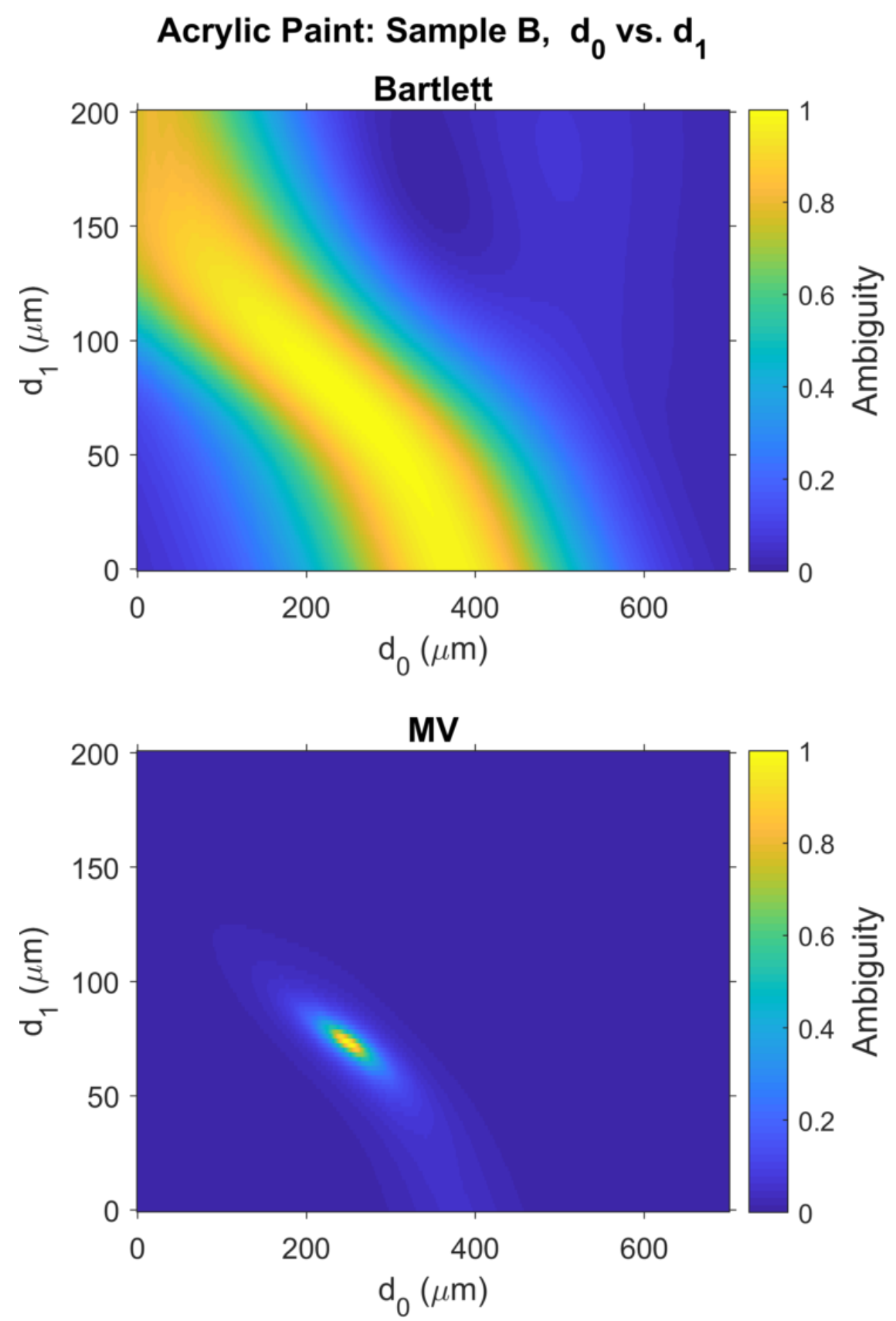

Figure 4.3.9: Sample B: Matched field ambiguity surfaces provide estimates for the thickness of the calibration layer, $d_{0}$, and acrylic paint layer, $d_{1}$.

Top panel: Bartlett processor global maximum at $d_{0}=248 \mu \mathrm{m}$ and $d_{1}=74 \mu \mathrm{m}$. Bottom panel: MV processor global maximum at $d_{0}=246 \mu \mathrm{m}$ and $d_{1}=74 \mu \mathrm{m}$. 

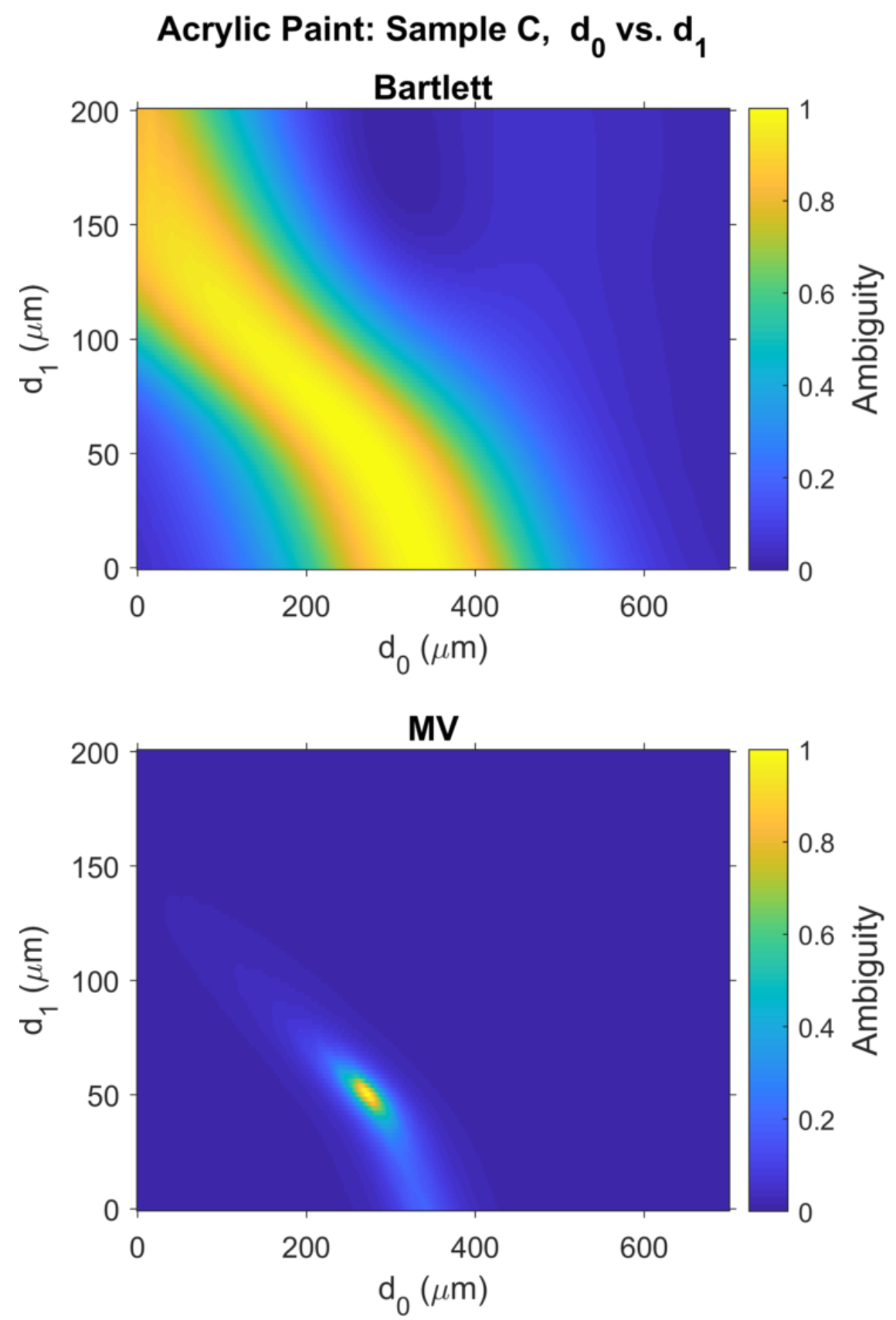

Figure 4.3.10: Sample C: Matched field ambiguity surfaces provide estimates for the thickness of the calibration layer, $d_{0}$, and acrylic paint layer, $d_{1}$.

Top panel: Bartlett processor global maximum at $d_{0}=272 \mu \mathrm{m}$ and $d_{1}=50 \mu \mathrm{m}$. Bottom panel: MV processor global maximum at $d_{0}=272 \mu \mathrm{m}$ and $d_{1}=50 \mu \mathrm{m}$. 

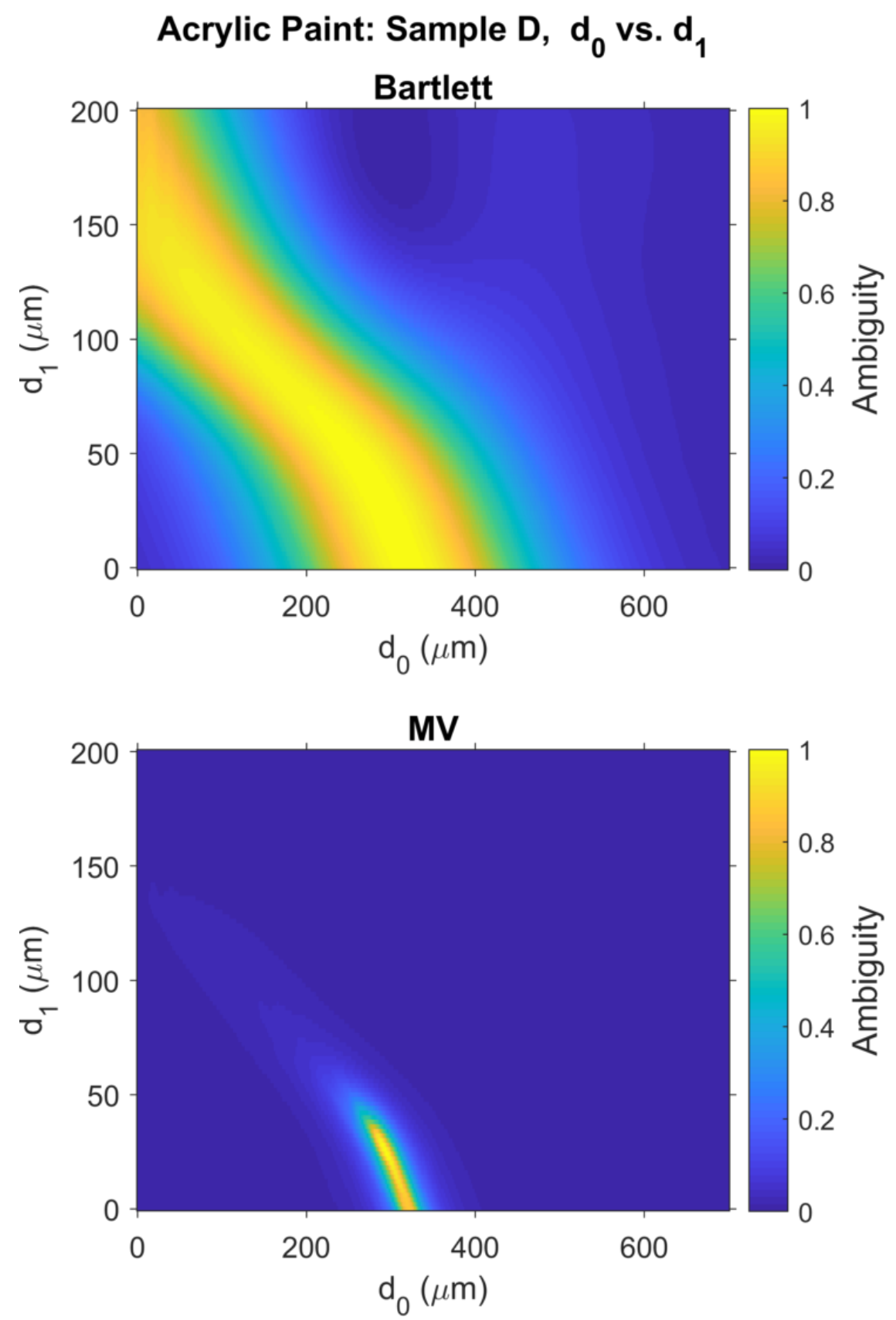

Figure 4.3.11: Sample D: Matched field ambiguity surfaces provide estimates for the thickness of the calibration layer, $d_{0}$, and acrylic paint layer, $d_{1}$.

Top panel: Bartlett processor global maximum at $d_{0}=292 \mu \mathrm{m}$ and $d_{1}=26 \mu \mathrm{m}$. Bottom panel: MV processor global maximum at $d_{0}=292 \mu \mathrm{m}$ and $d_{1}=26 \mu \mathrm{m}$. 

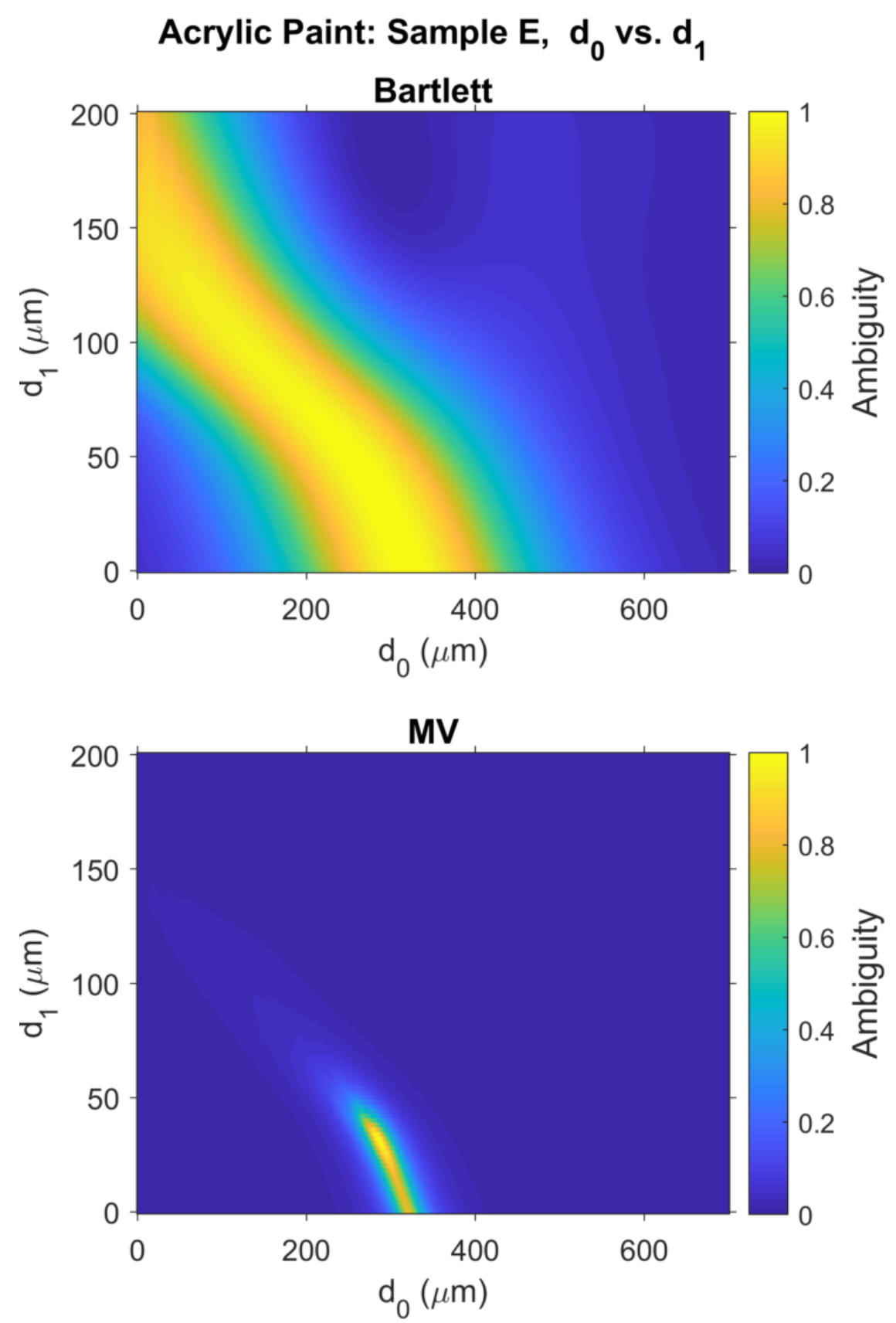

Figure 4.3.12: Sample E: Matched field ambiguity surfaces provide estimates for the thickness of the calibration layer, $d_{0}$, and acrylic paint layer, $d_{1}$.

Top panel: Bartlett processor global maximum at $d_{0}=288 \mu \mathrm{m}$ and $d_{1}=30 \mu \mathrm{m}$. Bottom panel: MV processor global maximum at $d_{0}=286 \mu \mathrm{m}$ and $d_{1}=32 \mu \mathrm{m}$. 

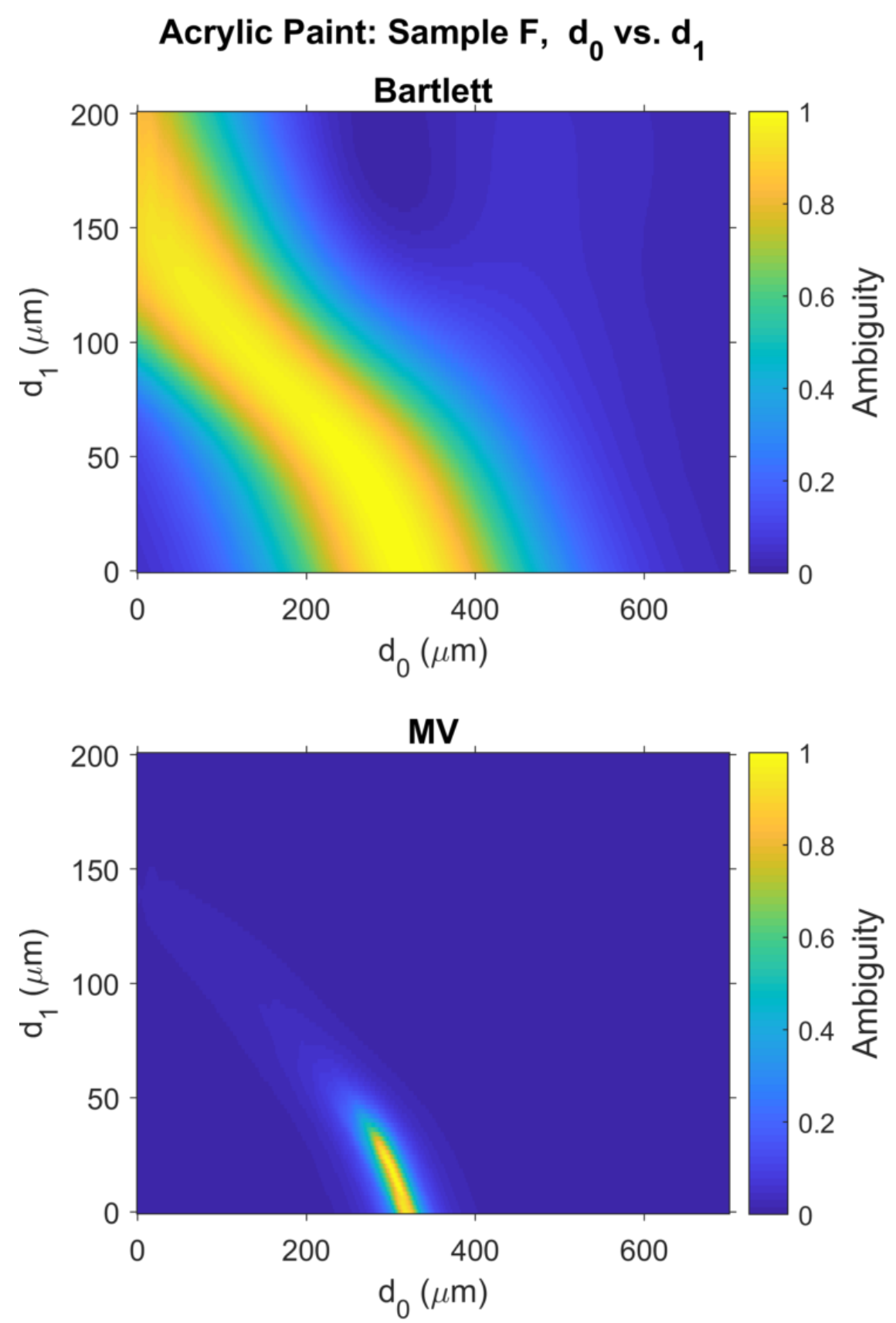

Figure 4.3.13: Sample F: Matched field ambiguity surfaces provide estimates for the thickness of the calibration layer, $d_{0}$, and acrylic paint layer, $d_{1}$.

Top panel: Bartlett processor global maximum at $d_{0}=298 \mu \mathrm{m}$ and $d_{1}=20 \mu \mathrm{m}$. Bottom panel: MV processor global maximum at $d_{0}=308 \mu \mathrm{m}$ and $d_{1}=12 \mu \mathrm{m}$. 
Table 4.3.2: Thickness estimates obtained from THz MFP with the Bartlett and MV objective functions for the experiment configuration shown in Figure 4.3.1. All of the differences between the THz MFP results and the CEM DT-156 Coating Thickness Tester measurements are within the uncertainty values for the Coating Thickness Tester.

\begin{tabular}{|c||c|c||c|c||}
\hline $\begin{array}{c}\text { Sample } \\
\text { ID }\end{array}$ & $\begin{array}{c}\text { Layer } \\
\text { ID }\end{array}$ & $\begin{array}{c}\text { CEM DT-156 } \\
(\mu m)\end{array}$ & $\begin{array}{c}\text { THz MFP Bartlett } \\
(\mu m)\end{array}$ & $\begin{array}{c}\text { THz MFP: MV } \\
(\mu m)\end{array}$ \\
\hline \hline A & $d_{0}$ & NA & 198 & 196 \\
\hline A & $d_{1}$ & $110 \pm 3.9$ & 112 & 112 \\
\hline \hline B & $d_{0}$ & NA & 248 & 246 \\
\hline B & $d_{1}$ & $72.0 \pm 3.0$ & 74 & 74 \\
\hline \hline C & $d_{0}$ & NA & 272 & 272 \\
\hline C & $d_{1}$ & $49.7 \pm 2.7$ & 50 & 292 \\
\hline \hline D & $d_{0}$ & NA & 292 & 266 \\
\hline D & $d_{1}$ & $28.4 \pm 3.2$ & 26 & 32 \\
\hline \hline E & $d_{0}$ & NA & 288 & 308 \\
\hline E & $d_{1}$ & $34.1 \pm 3.1$ & 30 & 12 \\
\hline \hline F & $d_{0}$ & NA & 298 & 20 \\
\hline F & $d_{1}$ & $16.2 \pm 2.1$ & & \\
\hline
\end{tabular}

$38 \mu m$ thinner than the paint layer for sample A. The difference of $50-38=12 \mu \mathrm{m}$ is likely due to minor differences in the thicknesses of the aluminum plates and/or minor positioning errors in the experiment configuration illustrated in Figure 4.3.1.

A visual comparison of the thickness data listed is Table 4.3.2 is provided in Figure 4.3.14. For each of the CEM DT-156 Coating Thickness Tester measurements listed in the table, the corresponding thickness estimates from THz MFP with the Bartlett and MV processors are plotted as blue circles and red squares, respectively. The dashed line represents an ideal case of equality of the Coating Thickness Tester measurement and the THz MFP thickness estimate. Thus, all of the measurement data from the 
THz MFP approach is in close agreement with the results from the CEM DT-156

Coating Thickness Tester. A small error is shown for the thinnest sample (sample F) as discussed above. 


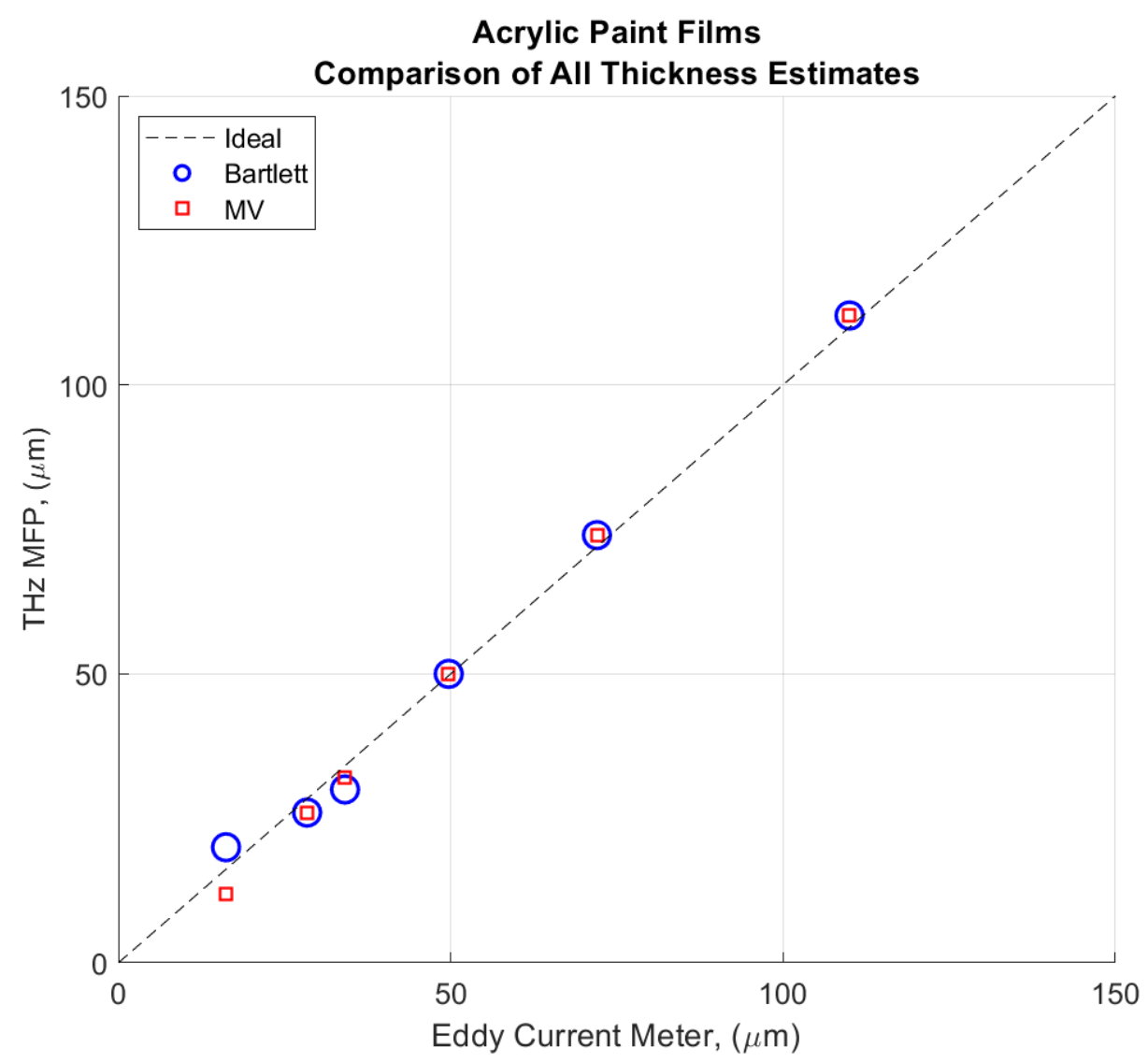

Figure 4.3.14: For each of the CEM DT-156 Coating Thickness Tester measurements listed in Table 4.3.2, the corresponding thickness estimates from $\mathrm{THz} \mathrm{MFP}$ with the Bartlett and MV processors are plotted as blue circles and red squares, respectively. The dashed line represents an ideal case of equality of the Coating Thickness Tester measurement and the $\mathrm{THz}$ MFP thickness estimate. Thus, most of the measurement data from the THz MFP approach is in close agreement conventional measurements, except for small errors for the thinnest sample (sample F). 


\section{Chapter 5}

\section{Conclusions and Future Work}

In recent years, THz sensing has emerged as an exciting new modality for NDE with potential for many applications in industrial quality control, bio-medical imaging and security screening. Deconvolution processing has often been used in the laboratory results reported in the $\mathrm{THz}$ scientific literature as a method to estimate the impulse response for layered materials. The TDOA between impulses can then be used with the group speed to calculate the layer thicknesses. Pulsed THz systems are commercially available with wide bandwidths that allow for resolution on the order of 100's of microns.

Two challenges for THz NDE of layered media were discussed in this dissertation. First, the practical implementation of $\mathrm{THz}$ systems for NDE in many applications will require fast scanning rates to make A-scans over large surfaces and/or many samples. The conventional deconvolution processing method for estimating the im-

pulse response is not well-suited to low SNR conditions that occur when measuring $\mathrm{THz}$ waveforms with short integration time. This method tends to amplify the noise and often results in artifacts that can create false positives when attempting to detect layer boundaries in the impulse response. Second, there is a desire to stretch the reso- 
lution limits for $\mathrm{THz}$ systems to perform NDE of surface coating layers that typically have thicknesses on the order of 10's of microns. In such thin film applications, the $\mathrm{THz}$ pulses reflected from the layer boundaries overlap on top of one another and the conventional TDOA cannot be used to calculate the thickness.

This work addresses these challenges by adapting physics-based signal processing methods that have traditionally been used for radar/sonar signal processing applications. A signal model was developed and used in the MLE to derive the matched filter for $\mathrm{THz}$ NDE applications, which was shown to be superior to the conventional deconvolution processing method in low SNR conditions. This was demonstrated with ROC curves that were created from $\mathrm{THz}$ measurements of layered polymer samples. Results were presented at an IEEE conference and documented in a 4-page conference paper [37]. Furthermore, the MFP methodology that has often appeared in the underwater acoustics literature was adapted to THz NDE of thin film layered media. This technique was applied to THz laboratory measurements of a thin air film embedded in thin films of polycarbonate, as well as with paint films of various thickness on an aluminum substrate. The accuracy of the measurements were validated with other measurement modalities, such as a Vernier caliper and eddy-current meter. Results were published in a peer-reviewed journal paper [38].

\subsection{Broader Impacts and Future Work}

To the author's knowledge this is the first time that MFP methods using the covariance matrix, the Bartlett processor, the Minimum variance processor and ambiguity plots have been applied to $\mathrm{THz}$ NDE. Thus, new and novel methods for $\mathrm{THz} \mathrm{NDE}$ have been introduced and proof of the potential of these concepts has been demon- 
strated using laboratory measurements in the NEAR-Lab at PSU. Building on the foundation of this research, future work could be done to further develop and expand MFP for THz NDE applications.

Additional work could be done to compute the theoretical resolution limit for MFP in $\mathrm{THz} \mathrm{NDE}$ of layered media. It is sometimes desirable to also determine the minimum accuracy that could be expected for a given measurement system and/or sample. The limitations on the accuracy of the parameter estimation can be evaluated using the Cramer-Rao lower bounds, which are expressed in terms of the elements of the inverse of the Fischer information matrix [33], [35], [63]. The elements of the Fischer information matrix are functions of the true values of the parameters, and therefore the minimum achievable accuracy depends on the particular sample under test. Formulation of the Cramer-Rao lower bounds for THz NDE applications is beyond the scope of this dissertation. Resources for further study of accuracy limitations in parameter estimation can be found in the literature [33], [35], [63].

It is important to note that the potential of MFP for THz NDE applications is not limited to the estimation of thin film thickness. For 3D chemical mapping of layered random media the parameter space may be expanded to include many more variables. $\mathrm{THz}$ scattering from a random rough surface can be characterized by the surface statistics, including the root mean square (rms) height of the surface, $h_{r m s}$ and the correlation length, $l_{c}[8],[64]-[68]$. THz scattering due to particles randomly distributed within a volume may be characterized by the permittivity of the background media, $\epsilon$, the permittivity of the scatterer, $\epsilon_{s}$. the scattering cross section of the scatterer, $\sigma_{s}$, the radius, $a$, of a hypothetical sphere surrounding each scatterer, and the fractional volume, $f_{v}$, of the scatterers within the background 
material [8], [69]-[73]. Thus, in non-dispersive random media the parameter vector could be written as

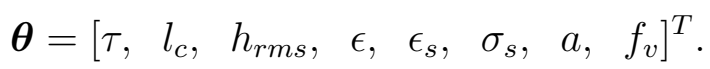

$\mathrm{THz}$ NDE of random media was performed by the author and was presented at the ISOT Conference in Seattle, WA in 2014 [74] and the IEEE International Symposium on Antennas and Propagation and North American Radio Science Meeting in Vancouver, BC Canada in 2015 [75]. In the future, the THz MFP methods that were applied to thin films in this dissertation could be applied to estimate the particle radius and/or fractional volume of scatterers embedded in a background medium.

For many $\mathrm{THz}$ applications the background media and/or the scattering media may be dispersive, in which case the Lorentz model is often used to describe the frequencydependent relative permittivity [58], [76]-[79] as

$$
\epsilon(f)=\epsilon_{\infty}+\sum_{p=1}^{P} \frac{\Delta \epsilon_{p} \omega_{p}^{2}}{\omega_{p}^{2}+2 j \omega \delta_{p}-\omega^{2}}
$$

where $\epsilon_{\infty}$ is the relative dielectric constant at the upper frequency limit, $\omega_{p}$ is the frequency of the $p$ th pole, $\Delta \epsilon_{p}$ is the change in the relative permittivity due to the $p$ th pole pair and $\delta_{p}$ is the damping coefficient of the pole. For example, to detect the propagation delay to a dispersive media with $P$ poles, the parameter vector may be written as follows,

$$
\boldsymbol{\theta}=\left[\tau, \Delta \epsilon_{1}, \ldots, \Delta \epsilon_{P}, \omega_{1}, \ldots, \omega_{P}, \quad \delta_{1}, \ldots, \delta_{P}\right]^{T}
$$


Finally, the techniques presented here could also be extended to $\mathrm{THz}$ synthetic aperture arrays using mathematical approaches in the MFP literature [49]. THz synthetic aperture imaging can be performed by raster scanning a monostatic THz emitter/sensor pair above the sample under test [50]-[53].

Thus, it is expected that this research will provide an important link for $\mathrm{THz}$ researchers to access and apply the robust methods that have been developed over several decades for other applications.

\subsection{Publications and Conference Presentations}

Below is a list of $\mathrm{THz}$ publications and conference presentations that have been authored or co-authored during the course of conducting this research.

- S. Schecklman and L. M. Zurk,Terahertz Imaging of Thin Film Layers with Matched Field Processing, Sensors 18, no. 10: 3547, October 2018.

- S. Schecklman, L. M. Zurk, and G. Kniffin, Terahertz Scattering from Contaminants Embedded in Textile Rope and Sling Materials, IEEE Symposium on Antennas and Propagation and North America Science Meeting, Session: Subsurface Remote Sensing, Vancouver, B.C., Canada, July 2015.

- S. Schecklman, G. P. Kniffin and L. M. Zurk, Terahertz Non-Destructive Evaluation of Textile Ropes and Slings, ISOT Converence, Seattle, WA, Nov. 2014 .

- S. Schecklman, G. P. Kniffin and L. M. Zurk, Terahertz Non-Destructive Evaluation of Layered Media with the Maximum Likelihood Estimator, ISOT 
Converence, Seattle, WA, Nov. 2014.

Prior to beginning this work the author performed $\mathrm{THz}$ research that resulted in a Master's Thesis [80], a peer-reviewed journal article [68], a book chapter [81] and several other THz publications [50]-[52], [57], [66], [67], [73], [82], [83]. In the field of underwater acoustics, the author has been lead author for 3 peer-reviewed journal papers [84]-[86] and authored or co-author of several other publications [87]-[90]. 


\section{Bibliography}

[1] D. Woolard, R. Brown, M. Pepper, and M. Kemp, "Terahertz frequency sensing and imaging: A time of reckoning future applications?" English, Proceedings of the IEEE, vol. 93, no. 10, pp. $1722-43,2005 / 10 /$.

[2] D. Dragoman and M. Dragoman, "Terahertz fields and applications," Progress in Quantum Electronics, vol. 28, no. 1, pp. 66 -, 2004.

[3] S. Mickan and X.-C. Zhang, "T-ray sensing and imaging," English, International Journal of High Speed Electronics and Systems, vol. 13, no. 2, pp. 601 -676, 2003.

[4] X.-C. Zhang, "Terahertz wave imaging: Horizons and hurdles," English, 21, vol. 47, UK, 2002, pp. $3667-77$.

[5] M. Kemp, P. Taday, B. Cole, J. Cluff, A. Fitzgerald, and W. Tribe, "Security applications of terahertz technology," English, Proceedings of SPIE - The International Society for Optical Engineering, vol. 5070, pp. 44 -52, 2003.

[6] A. G. Davies, A. D. Burnett, W. Fan, E. H. Linfield, and J. E. Cunningham, "Terahertz spectroscopy of explosives and drugs," Materials Today, vol. 11, no. 3, pp. 18-26, Mar. 2008.

[7] K. Kawase, Y. Ogawa, Y. Watanabe, and H. Inoue, "Non-destructive terahertz imaging of illicit drugs using spectral fingerprints," English, Optics Express, vol. 11, no. 20, pp. $2549-2554,2003$. 
[8] K.-E. Peiponen, A. Zeitler, and M. Kuwata-Gonokami, Terahertz Spectroscopy and Imaging. Springer, 2013.

[9] Y.-C. Shen, P. F. Taday, D. A. Newnham, M. C. Kemp, and M. Pepper, "3d chemical mapping using terahertz pulsed imaging," English, vol. 5727, San Jose, CA, United states, 2005, pp. $24-31$.

[10] J. A. Zeitler, Y. Shen, C. Baker, P. F. Taday, M. Pepper, and T. Rades, "Analysis of coating structures and interfaces in solid oral dosage forms by three dimensional terahertz pulsed imaging," Journal of Pharmaceutical Sciences, vol. 96 , no. 2 , pp. 330-340, 2007.

[11] J. A. Zeitler and L. F. Gladden, "In-vitro tomography and non-destructive imaging at depth of pharmaceutical solid dosage forms," European Journal of Pharmaceutics and Biopharmaceutics, vol. 71, no. 1, pp. 2-22, 2009.

[12] T. Yasui, T. Yasuda, T. Iwata, and T. Araki, "Simultaneous measurement of thickness and drying process of paint film by terahertz electromagnetic pulse," English, vol. vol.2, Piscataway, NJ, USA, 2003, 480 vol.2 -.

[13] T. Yasui, T. Yasuda, K.-I. Sawanaka, and T. Araki, "Terahertz paintmeter for noncontact monitoring of thickness and drying progress in paint film," English, Applied Optics, vol. 44, no. 32, pp. 6849 -6856, 2005.

[14] T. Yasuda, T. Iwata, T. Araki, and T. Yasui, "Improvement of minimum paint film thickness for thz paint meters by multiple-regression analysis," English, Applied Optics, vol. 46, no. 30, pp. 7518 -26, 2007.

[15] T. Kurabayashi, S. Sakai, and K. Fujino, "Sub-terahertz imaging of a painted steel," English, Piscataway, NJ, USA, 2010, 2 pp.-- 
[16] I. TeraView, "Using 3-dimensional terahertz pulsed imaging to analyse automotive coatings," TeraView Limited, Tech. Rep., 2013.

[17] M. Yamashita, T. Kiwa, M. Tonouchi, and K. Kawase, "Development of laserterahertz emission microscope for inspecting the electrical faults in semiconductor devices," in Conference on Lasers and Electro-Optics, Optical Society of America, 2004, CThN5.

[18] C. Jansen, S. Wietzke, O. Peters, M. Scheller, N. Vieweg, M. Salhi, N. Krumbholz, C. Jordens, T. Hochrein, and M. Koch, "Terahertz imaging: Applications and perspectives," Applied Optics, vol. 49, no. 19, E48 -E57, 2010.

[19] J. Jackson, M Mourou, J. Whitaker, I. Duling III, S. Williamson, M Menu, and G. Mourou, "Terahertz imaging for non-destructive evaluation of mural paintings," Optics Communications, vol. 281, no. 4, pp. 527-532, 2008.

[20] J. Jackson, J. Labaune, G. Mourou, L. D'Alessandro, A. Whyte, and M. Menu, "Pulsed terahertz investigation of corroded and mineralized copper alloy historical artifacts," English, Houston, TX, United states, 2011.

[21] K. Fukunaga, Y. Ogawa, S. Hayashi, and I. Hosako, "Application of terahertz spectroscopy for character recognition in a medieval manuscript," IEICE Electronics Express, vol. 5, no. 7, pp. $223-228,2008$.

[22] K. Fukunaga, Y. Ogawa, S. Hayashi, and I. Hosako, "Terahertz imaging for analysis of historic paintings and manuscripts," in 200833 rd International Conference on Infrared, Millimeter and Terahertz Waves, Sep. 2008, pp. 1-3.

[23] K. Fukunaga and M. Picollo, "Terahertz spectroscopy applied to the analysis of artists materials," Applied Physics A, vol. 100, no. 3, pp. 591-597, 2010. 
[24] J. Labaune, J. Jackson, S. Pages-Camagna, M. Menu, and G. Mourou, "Terahertz investigation of egyptian artifacts," English, Rome, Italy, 2010.

[25] R. M. Woodward, B. E. Cole, V. P. Wallace, R. J. Pye, D. D. Arnone, E. H. Linfield, and M. Pepper, "Terahertz pulse imaging in reflection geometry of human skin cancer and skin tissue," Physics in Medicine and Biology, vol. 47, no. 21 , pp. $3853-3863,2002$.

[26] V. P. Wallace, A. J. Fitzgerald, E. Pickwell, R. J. Pye, P. F. Taday, N. Flanagan, and H. Thomas, "Terahertz pulsed spectroscopy of human basal cell carcinoma," Applied Spectroscopy, vol. 60, no. 10, pp. 1127 -1133, 2006.

[27] J. Zuo, Z.-W. Zhang, J. He, L.-L. Zhang, K.-J. Mu, and C.-L. Zhang, "The experimental research of leaf water content using terahertz time-domain spectroscopy," English, vol. 8195, Beijing, China, 2011, Chinese Society of Astronautics (CAS) - .

[28] T. Iwata, S. Yoshioka, S. Nakamura, and et al., "Prediction of the thickness of a thin paint film by applying a modified partial-least-squares-1 method to data obtained in terahertz reflectometry," JOURNAL OF INFRARED MILLIMETER AND TERAHERTZ WAVES, vol. 34, pp. 646-659, 2013.

[29] K. Su, Y.-C. Shen, and J. Zeitler, "Terahertz sensor for non-contact thickness and quality measurement of automobile paints of varying complexity," English, IEEE Transactions on Terahertz Science and Technology, vol. 4, no. 4, pp. 432 $-9,2014$.

[30] J. L. M. van Mechelen, A. B. Kuzmenko, and H. Merbold, "Stratified dispersive model for material characterization using terahertz time-domain spectroscopy," OPTICS LETTERS, vol. 39, pp. 3853-3856, 2014. 
[31] F. Le Chevalier, Principles of Radar and Sonar Signal Processing. Artech House, Inc. Boston, MA, 2002.

[32] M. I. Skolnik, Introduction to Radar Systems, 3rd Ed. 3rd. McGraw-Hill Inc., New York, NY, 2001.

[33] S. Kay, Fundamentals of Statistical Signal Processing: : Vol. 1 Estimation Theory. Upper Saddle River, NJ: Prentice-Hall, Inc., 1993.

[34] L. N. F. A. Tolstoy O. Diachok, "Acoustic tomography via matched field processing," J. Acoust. Soc. Am., vol. 89, no. 3, Mar. 1991.

[35] A. Baggeroer, W. Kuperman, and P. Mikhalevsky, "An overview of matched field methods in ocean acoustics," English, IEEE Journal of Oceanic Engineering, vol. 18, no. 4, pp. $401-24,1993$.

[36] A. Tolstoy, Matched Field Processing for Underwater Acoustics. World Scientific Publishing Company, 1993.

[37] S. Schecklman, G. Kniffin, and L. Zurk, "Terahertz non-destructive evaluation of layered media with the maximum likelihood estimator," in 2014 International Symposium on Optomechatronic Technologies, 2014, pp. 81 -85.

[38] S. Schecklman and L. M. Zurk, "Terahertz imaging of thin film layers with matched field processing," MDPI Sensors Journal, vol. 18, no. 10, p. 3547, Oct. 2018.

[39] T. Yasuda, T. Yasui, T. Araki, and E. Abraham, "Real-time two-dimensional terahertz tomography of moving objects," Optics Communications, vol. 267, no. 1, pp. 128-136, Nov. 2006. 
[40] E. Pickwell, B. Cole, A. Fitzgerald, M. Pepper, and V. Wallace, "In vivo study of human skin using pulsed terahertz radiation," Phys. Med. Biol., vol. 49, no. 9, Apr. 2004.

[41] Y. Chen, S. Huang, and E. Pickwell-Macpherson, "Frequency-wavelet domain deconvolution for terahertz reflection imaging and spectroscopy," Opt. Express, vol. 18, no. 2, pp. 1177-1190, 2010.

[42] E. Parrott, S. Sy, T. Blu, B. Wallace, and E. Pickwell-Macpherson, "Terahertz pulsed imaging in vivo: Measurements and processing methods," Journal of Biomedical Optics, vol. 16, no. 10, Oct. 2011.

[43] D. Gingras, P. Gerstoft, and N. Gerr, "Electromagnetic matched-field processing: Basic concepts and tropospheric simulations," English, IEEE Transactions on Antennas and Propagation, vol. 45, no. 10, pp. 1536 -45, 1997.

[44] F. B. Jensen, W. A. Kuperman, M. B. Porter, and H. Schmidt, Computational Ocean Acoustics (Modern Acoustics and Signal Processing), 2nd ed. Springer, 2011.

[45] H. Bucker, "Use of calculated sound fields and matched-field detection to locate sound sources in shallow water," English, Journal of the Acoustical Society of America, vol. 59, no. 2, pp. $368-73,1976$.

[46] Z.-H. Michalopoulou and M. Porter, "Matched-field processing for broad-band source localization," English, IEEE Journal of Oceanic Engineering, vol. 21, no. 4, pp. $384-92,1996$.

[47] Z.-H. Michalopoulou, "Robust multi-tonal matched-field inversion: A coherent approach," English, Journal of the Acoustical Society of America, vol. 104, no. 1, pp. $163-70,1998$. 
[48] P. N. Martin Siderius Peter Gerstoft, "Broadband geoacoustic inversion from sparse data using genetic algorithms," Journal of Computational Acoustics, vol. 06, no. 1, pp. 117-134, Mar. 1998.

[49] S. E. Dosso and M. J. Wilmut, "Maximum-likelihood and other processors for incoherent and coherent matched-field localization," The Journal of the Acoustical Society of America, vol. 132, no. 4, pp. 2273 -2285, Oct. 2012.

[50] L. Zurk, S. Henry, and S. Schecklman, "Terahertz spectral imaging using correlation processing," English, Piscataway, NJ, USA, 2012, 2 pp. - .

[51] S. C. Henry, L. M. Zurk, S. Schecklman, and D. D. Duncan, "Three-dimensional broadband terahertz synthetic aperture imaging," Optical Engineering, vol. 51, no. 9, pp. $091603-1,2012$.

[52] S. Henry, L. Zurk, and S. Schecklman, "Terahertz spectral imaging using correlation processing," English, IEEE Transactions on Terahertz Science and Technology, vol. 3, no. 4, pp. $486-93,2013$.

[53] G. Kniffin and L. M. Zurk, "Parabolic equation methods for terahertz 3-d synthetic aperture imaging," IEEE Transactions on Terahertz Science and Technology, vol. PP, no. 99, pp. 1-9, 2016.

[54] L. Duvillaret, F. Garet, and J.-L. Coutaz, "A reliable method for extraction of material parameters in terahertz time-domain spectroscopy," English, IEEE Journal of Selected Topics in Quantum Electronics, vol. 2, no. 3, pp. 739 -46, 1996.

[55] T. D. Dorney, J. L. Johnson, J Van Rudd, R. G. Baraniuk, W. W. Symes, and D. M. Mittleman, "Terahertz reflection imaging using kirchhoff migration," Optics letters, vol. 26, no. 19, pp. 1513-1515, 2001. 
[56] E. Parrott, J. Zeitler, J. McGregor, S. Oei, H. Unalan, S. Tan, W. I. Milne, J. Tessonnier, R. Schlogl, and L. Gladden, "Understanding the dielectric properties of heat-treated carbon nanofibers at terahertz frequencies: A new perspective on the catalytic activity of structured carbonaceous materials," J. Phys. Chem, 2009.

[57] G. Kniffin, S. Schecklman, J. Chen, S. Henry, L. Zurk, B. Pejcinovic, and A. Timchenko, "Measurement and modeling of terahertz spectral signatures from layered material," English, vol. 7687, USA, 2010, 768708 (11 pp.) -.

[58] G. Kniffin and L. Zurk, "Model-based material parameter estimation for terahertz reflection spectroscopy," IEEE Transactions on THz, 2012.

[59] E. Hecht, Optics, 4th Edition. Addison-Wesley, 2001.

[60] Y.-S. Jin, G.-J. Kim, and S.-G. Jeon, "Terahertz dielectric properties of polymers," English, Journal of the Korean Physical Society, vol. 49, no. 2, pp. 513 $-17,2006$.

[61] Dupli-Color, https://www.duplicolor.com/product/perfect-match-premium-automotivepaint, 2019.

[62] C. D.-.D. P. Meter, http://cem-instruments.com/en/Product/type2/id/788, 2019.

[63] H. L. Van Trees, Detection, Estimation, and Modulation Theory, Optimum Array Processing. John Wiley \& Sons, 2004.

[64] L. M. Zurk, B. Orlowski, D. P. Winebrenner, E. I. Thorsos, M Leahy-Hoppa, and M. R. Hayden, "Terahertz scattering from granular material," Journal of the Optical Society of America B: Optical Physics, vol. 29, no. 9, 2238 2243, 2007. 
[65] S. Schecklman and L. M Zurk, "Terahertz scattering from random rough surfaces," in Progress in Electromagnetics Research Symposium (PIERS), Boston, MA, Jul. 2008.

[66] S. Henry, S. Schecklman, G. Kniffin, L. Zurk, and A. Chen, "Measurement and modeling of rough surface effects on terahertz spectroscopy," English, vol. 7601, San Francisco, CA, United states, 2010, The Society of Photo-Optical Instrumentation Engineers (SPIE) - .

[67] G. Sundberg, L. Zurk, S. Schecklman, and S. Henry, "Modeling rough-surface and granular scattering at terahertz frequencies using the finite-difference timedomain method," English, IEEE Transactions on Geoscience and Remote Sensing, vol. 48, no. 10, pp. $3709-19,2010$.

[68] S. Schecklman, L. M. Zurk, S. Henry, and G. P. Kniffin, "Terahertz material detection from diffuse surface scattering," Journal of Applied Physics, vol. 109, no. 9, 094902, p. 094902, 2011.

[69] L. M. Zurk, B. Jouni, F. Farahbakhshian, D. Winebrenner, E. Thorsos, M. Leahy-Hoppa, and L. Hayden, "Calculation of scattering from polyethylene particles compared with terahertz measurements," Progress in Electromagnetic Research Sym, Boston, MA, 2006.

[70] L. M. Zurk, B. Orlowski, G. Sundberg, Z. Zhou, and A. Chen, "Terahertz scattering from a rough granular surface," English, IEEE Antennas and Propagation Society, APS International Symposium (Digest), pp. 4929 -4932, 2007.

[71] L. Zurk, B. Orlowski, G. Sundberg, Z. Zhou, and A. Chen, "Terahertz scattering from a rough granular surface," English, Piscataway, NJ, USA, 2008, pp. 4929 -32 . 
[72] M. H. Arbab, A. Chen, E. I. Thorsos, D. P. Winebrenner, and L. M. Zurk, "Effect of surface scattering on terahertz time domain spectroscopy of chemicals," Proceedings of SPIE The International Society for Optical Engineering, vol. 6893, pp. $68930-, 2008$.

[73] K. Nam, L. Zurk, and S. Schecklman, "Modeling terahertz diffuse scattering from granular media using radiative transfer theory," English, Progress In Electromagnetics Research B, vol. 38, pp. 205 -23, 2012.

[74] S. Schecklman, G. Kniffin, and L. Zurk, "Terahertz non-destructive evaluation of textile ropes and slings," English, Los Alamitos, CA, USA, 2014, pp. 86 -90.

[75] S. Schecklman, L. M. Zurk, and G. Kniffin, "Terahertz scattering from contaminants embedded in textile rope and sling materials," in IEEE International Symposium on Antennas and Propagation and North American Radio Science Meeting in Vancouver BC Canada, Jul. 2015.

[76] K. Yamamoto, M. Yamaguchi, F. Miyamaru, M. Tani, M. Hangyo, T. Ikeda, A. Matsushita, K. Koide, M. Tatsuno, and Y. Minami, "Noninvasive inspection of c-4 explosive in mails by terahertz time-domain spectroscopy," English, Japanese Journal of Applied Physics, Part 2 (Letters), vol. 43, no. 3B, pp. 414 $-17,2004$.

[77] J. Bjarnason, E. Brown, and T. Korter, "Comparison of the thz absorption feature in lactose to related saccharides," English, 1, vol. 6549, USA, 2007, pp. $65490-1$.

[78] O. Ahmed, M. Swillam, M. Bakr, and X. Li, "Efficient optimization approach for accurate parameter extraction with terahertz time-domain spectroscopy," English, Journal of Lightwave Technology, vol. 28, no. 11, pp. 1685 -92, 2010. 
[79] A. Roggenbuck, H. Schmitz, A. Deninger, I. Mayorga, J. Hemberger, R. Gusten, and M. Gruninger, "Coherent broadband continuous-wave terahertz spectroscopy on solid-state samples," English, New Journal of Physics, vol. 12, no. 4, 043017 (13 pp.) -, 2010.

[80] S. Schecklman, "Terahertz scattering from random rough surfaces," Master's thesis, Portland State University, 2009.

[81] L. M. Zurk and S. Schecklman, "Terahertz spectroscopy and imaging," in, K. E. Peiponen, J. A. Zeitler, and M. Kuwata-Gonokami, Eds. Springer-Verlag Berlin Heidelberg, 2013, ch. Chp. 5, Terahertz Scattering.

[82] L. Zurk, G. Sundberg, S. Schecklman, Z. Zhou, A. Chen, and E. Thorsos, "Scattering effects in terahertz reflection spectroscopy," English, vol. 6949, Orlando, FL, United states, 2008.

[83] L. Zurk, S. Henry, S. Schecklman, and D. Duncan, "Physics-based processing for terahertz reflection spectroscopy and imaging," English, vol. 7854, Beijing, China, 2010, The Society of Photo-Optical Instrumentation Engineers (SPIE); Chinese Optical Society (COS).

[84] S. Schecklman, M. Siderius, and D. Tornquist, "Computing the effect of sound on the marine environment by the adaptive mesh refinement method," English, Piscataway, NJ, USA, 2010, 6 pp. - .

[85] S. Schecklman, N. Laws, L. Zurk, and M. Siderius, "A computational method to predict and study underwater noise due to pile driving," English, Journal of the Acoustical Society of America, vol. 138, no. 1, pp. 258 -66, 2015. 
[86] S. Schecklman and L. M. Zurk, "Striation processing of data from the 2013 target and reverberation experiment (trex13)," IEEE Journal of Oceanic Engineering, 2016.

[87] L. M. Zurk, H. H. Ou, S. Schecklman, and A. Lutwak, "Acoustic monitoring of marine conservation areas," Marine Technology Society Journal, vol. 48, no. 6, pp. 21-32, 2014.

[88] S. Schecklman and L. Zurk, "Extraction of striations from continuous active sonar (cas) data," English, Piscataway, NJ, USA, 2015, 7 pp. - .

[89] S. Schecklman and L. M. Zurk, "Striation processing of continuously active sonar data," English, Journal of the Acoustical Society of America, vol. 137, no. 4, 2438 (1 pp.) -, 2015.

[90] L. M. Zurk, D. Rouseff, and S. Schecklman, "Exploitation of frequency information in continuous active sonar," PROCEEDINGS of the 22nd International Congress on Acoustics, Buenos Aires, Argentina, September 5-9, 2016, 2016.

[91] C. A. Balanis, Advanced Engineering Electromagnetics. John Wiley and Sons, 1989. 


\section{Appendix A}

\section{Propagation Model for Plane Waves in Layered Media}

\section{A.1 Introduction}

The MFP algorithm requires a forward propagation model to generate replicas of the $\mathrm{THz}$ spectrum that can be later compared with the measured THz spectrum by an objective function. This appendix describes the propagation model used to simulate the sample's transfer function, $H_{s}(f)$. Parameterization of the model in terms of the parameter vector, $\boldsymbol{a}$, will be discussed at the end of this section.

For many samples of interest (e.g. laminated composites, painted surfaces, etc.) can be approximated as a stack of plane-parallel layers. If the sample is within the focal depth of both the transmit and receive lenses of the THz-TDS system, then the propagation of the $\mathrm{THz}$ waves may be modeled as plane waves propagating within the stack of layers.

The composite reflection coefficient shown here is often available in advanced electromagnetics text books [91], but is provided here because of its relevance to understanding the frequency-dependent interference pattern that can be exploited for $\mathrm{THz}$ 
NDE of layered media. It is important to note, that for the pulsed $\mathrm{THz}$ systems that were introduced in Section 1.2, the assumption of plane wave propagation is only valid within the focal depth of the $\mathrm{THz}$ lens.

Here, it is assumed that $\mathrm{THz}$ NDE system is configured for bistatic measurement from a smooth planar layer sample, that is oriented to be at normal incidence. In this configuration, the reflection coefficient is independent of the polarization of the plane waves. For more complicated (bi-static) measurement geometries, the polarization of the $\mathrm{THz}$ beam would be relevant and the additional information provided by measurements made with two polarizations could possibly be exploited with MFP to provide more accuracy in the thickness estimate. In this dissertation, monostatic plane wave interaction with planar layered samples will be assumed. The more complicated cases of bistatic measurements with multiple polarizations remains a topic for future work.

\section{A.2 Transfer Matrix Model}

In this section, the basic components of a matrix model for multiple reflections with a parallel stack of an arbitrary number layers will be presented. A complete derivation is available in the literature [59].

A single layer of thickness, $d$, with smooth parallel boundaries is assumed. The top

boundary is denoted I and bottom boundary is denoted II. Figure 9.49 in [59] shows a detailed diagram of the layer geometry.

For a plane wave incident on the layer, the electric an magnetic fields at the top 
boundary, $E_{I}$ and $H_{I}$, are related to the fields at the bottom side of the layer, $E_{I I}$ and $H_{I I}$, by the following matrix equation,

$$
\left[\begin{array}{c}
E_{I} \\
H_{I}
\end{array}\right]=M \times\left[\begin{array}{c}
E_{I I} \\
H_{I I}
\end{array}\right]
$$

where the matrix, $M$ is

$$
M=\left[\begin{array}{cc}
\cos \left(k_{0} \tilde{n}_{1} h_{1}\right) & j \sin \left(k_{0} \tilde{n}_{1} h_{1}\right) / Y_{1} \\
j Y_{1} \sin \left(k_{0} \tilde{n}_{1} h_{1}\right) & \cos \left(k_{0} \tilde{n}_{1} h_{1}\right)
\end{array}\right]
$$

where $j=\sqrt{-1}, Y_{1}$ is the admittance of the medium in layer 1 , and $k_{0}=(2 \pi f) / c$ is the wave number in free space and $\tilde{n}_{1}$ is the complex index of refraction for layer 1. The complex index of refraction for each layer, $\tilde{n}_{q}$, is frequency-dependent and can be expressed as

$$
\tilde{n}_{q}(f)=n_{q}(f)+j \kappa_{q}(f)
$$

The real part of the refractive index, $n_{q}(f)$, accounts for dispersion and the extinction coefficient, $\kappa_{q}(f)$, accounts for attenuation withing the layer, $q$. The signal processing methods for determining the complex index of refraction of a sample material from an independent THz TDS measurement are already documented in the literature [3], [8] and therefore will not be discussed here.

In Eq. A.2.2, $h_{1}$ is the propagation distance inside layer 1 given by,

$$
h_{1}=d_{1} \cos \left(\phi_{i I I}\right) \text {. }
$$


where $\phi_{i I I}$ is the angle of propagation within the layer, 1, relative to the surface normal, due to Snell's law, and $d_{1}$ is the thickness of layer 1. Thus, for normal incidence, Eq. A.2.4 simplifies to $h_{1}=d_{1}$.

In general, if the sample consists of $Q$ layers, then the fields at the first and last boundaries are given by

$$
\left[\begin{array}{c}
E_{I} \\
H_{I}
\end{array}\right]=M_{I} \times M_{I I} \times M_{I I I} \times \ldots \times M_{q} \times\left[\begin{array}{c}
E_{q+1} \\
H_{q+1}
\end{array}\right],
$$

or

$$
\left[\begin{array}{c}
E_{I} \\
H_{I}
\end{array}\right]=\mathscr{M} \times\left[\begin{array}{c}
E_{q+1} \\
H_{q+1}
\end{array}\right]
$$

where the characteristic matrix of the entire layer stack, $\mathscr{M}$ is computed as the matrix product (in proper sequence) of each of the $2 \times 2$ matrices for each layer. Thus, the final characteristic matrix is also a $2 \times 2$ matrix given by

$$
\mathscr{M}=M_{1} \times M_{2} \times M_{3} \times \ldots \times M_{q}=\left[\begin{array}{cc}
m_{11} & m_{12} \\
m_{21} & m_{22}
\end{array}\right] .
$$

It can be shown that the sample's transfer function, $H_{s}(f)$ for the $\mathrm{THz}$ measurement with the sample composed of plane parallel layers can be expressed as [59] 


$$
H_{s}(f)=\frac{Y_{0} m_{11}+Y_{0} Y_{s} m_{12}-m_{21}-Y_{s} m_{22}}{Y_{0} m_{11}+Y_{0} Y_{s} m_{12}+m_{12}+Y_{s} m_{22}} .
$$

In Equation A.2.8, $Y_{0}$ and $Y_{s}$ are the admittance of the infinite half-space media above and below the layer stack. The specific details and formulas for computing the admittance are available in [59]. 


\section{Appendix B}

\section{MFP Results for Air Film in Polycarbonate}

This appendix contains all the results of the experiments that were described in Section 4.2 as illustrated in Figure 4.2.1.

Covariance Matrices for each sample (A-D) are shown in Section B.1.

Ambiguity surfaces for each sample are shown in Section B.2.

\section{B.1 Sample Covariance Matrices}



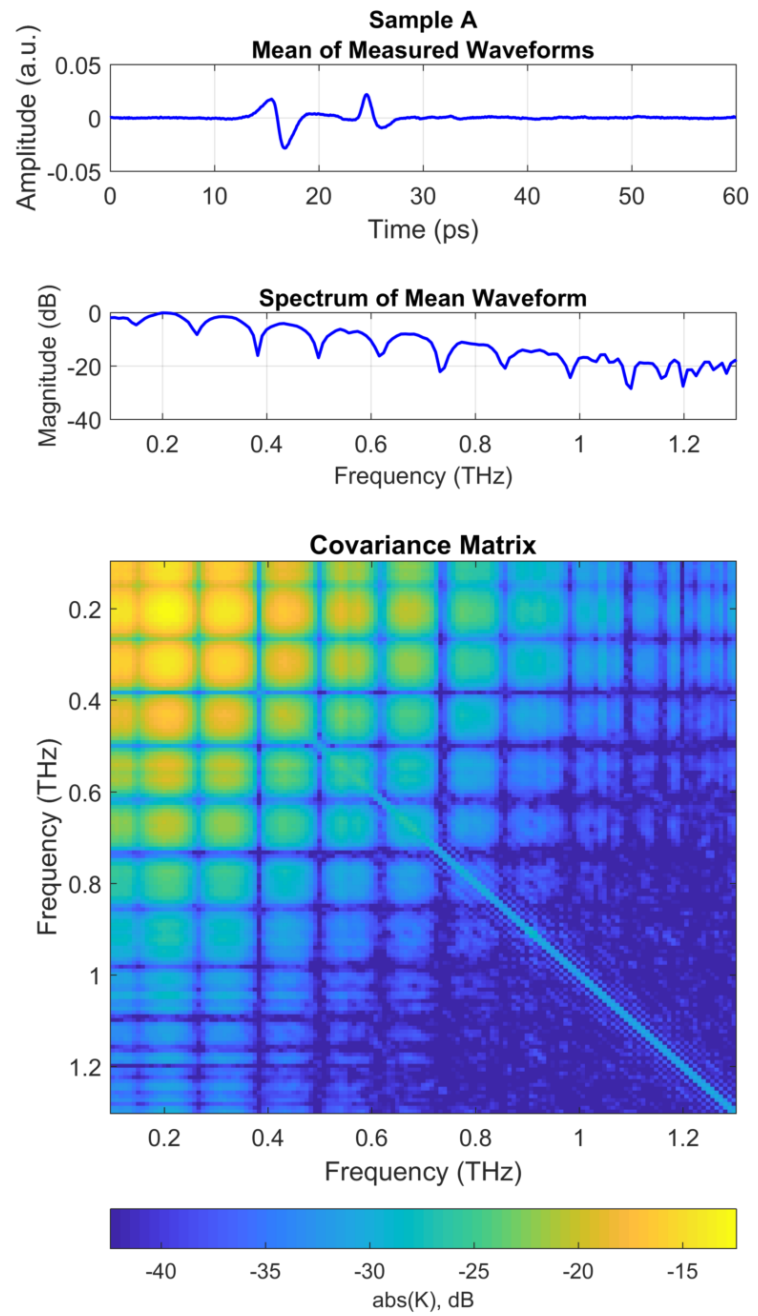

Figure B.1.1: Measured data for Sample A. Top Panel: Mean of measured THz waveforms. Note that reflected $\mathrm{THz}$ pulse 1 (illustrated in Figure 4.2.1) arrives at approximately $15 \mathrm{ps}$, and pulses 2 and 3 overlap with one another at approximately 25 ps. Middle Panel: Spectrum of mean waveform in the top panel. Bottom Panel: Covariance matrix computed with Eq. 3.3.9 using the spectrum of each of the 300 measured waveforms. 

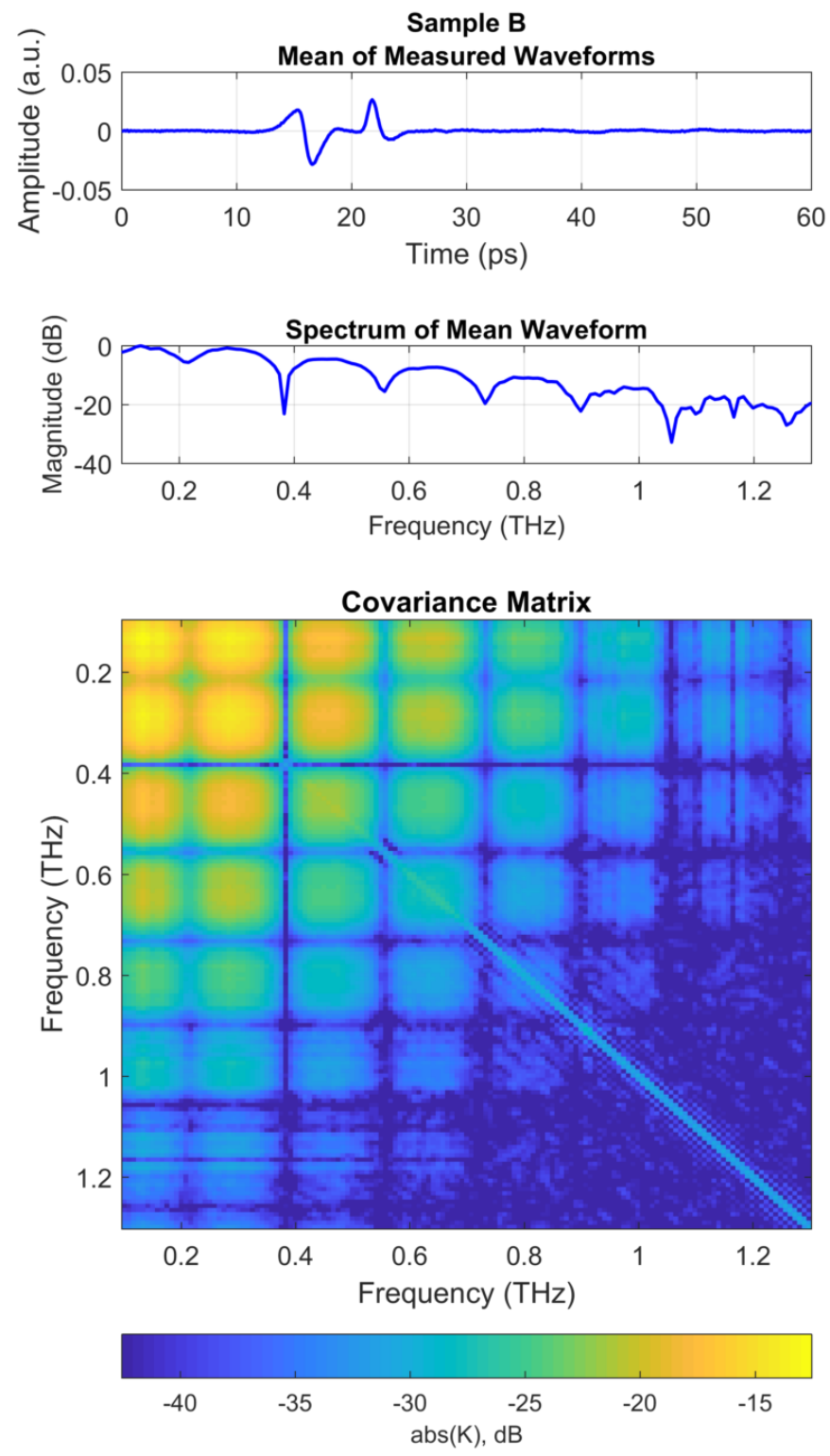

Figure B.1.2: Measured data for Sample B. Top Panel: Mean of measured THz waveforms. Middle Panel: Spectrum of mean waveform in the top panel. Bottom Panel: Covariance matrix computed with Eq. 3.3.9 using the spectrum of each of the 300 measured waveforms. 

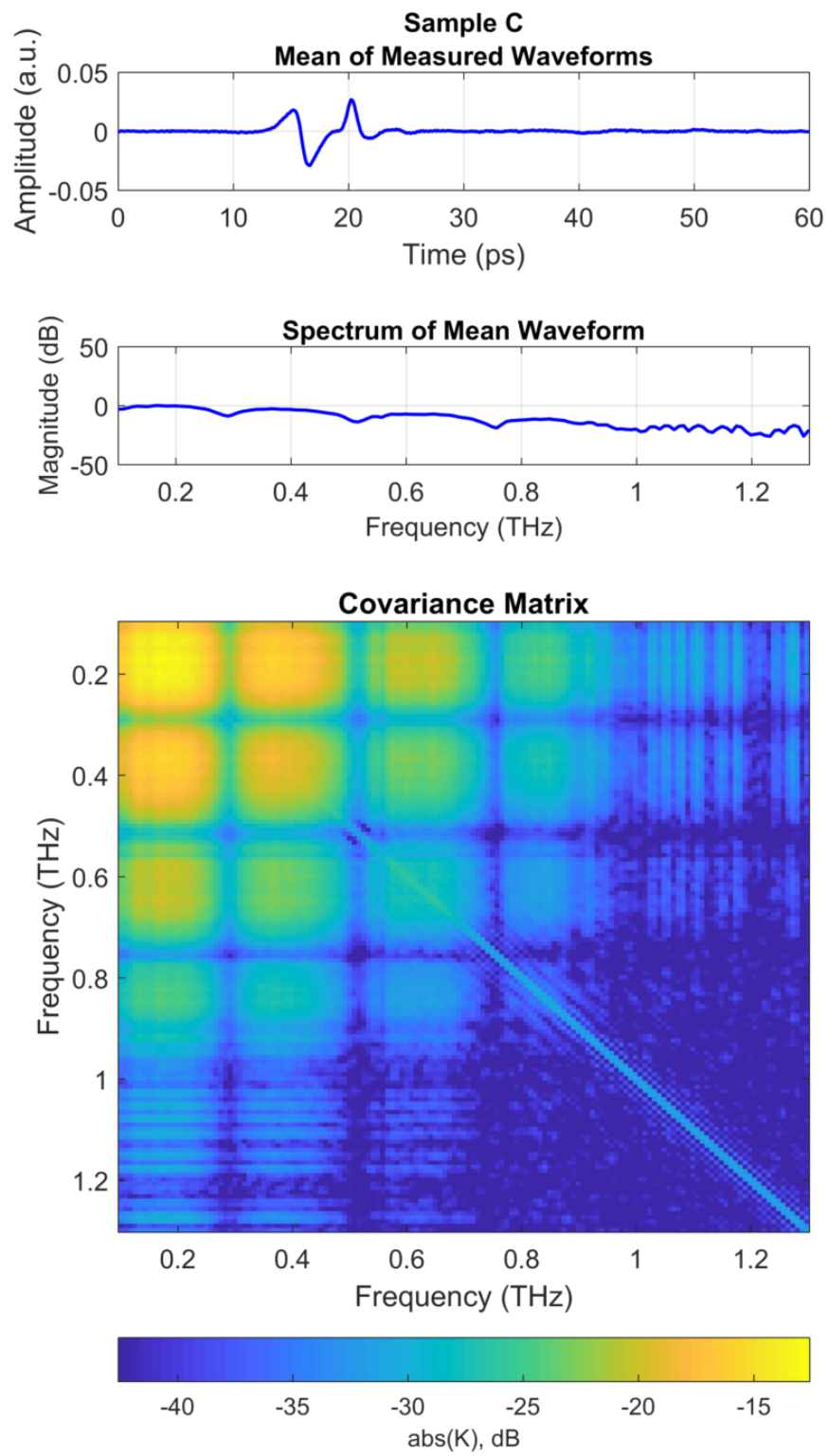

Figure B.1.3: Measured data for Sample C. Top Panel: Mean of measured THz waveforms. Middle Panel: Spectrum of mean waveform in the top panel. Bottom Panel: Covariance matrix computed with Eq. 3.3.9 using the spectrum of each of the 300 measured waveforms. 

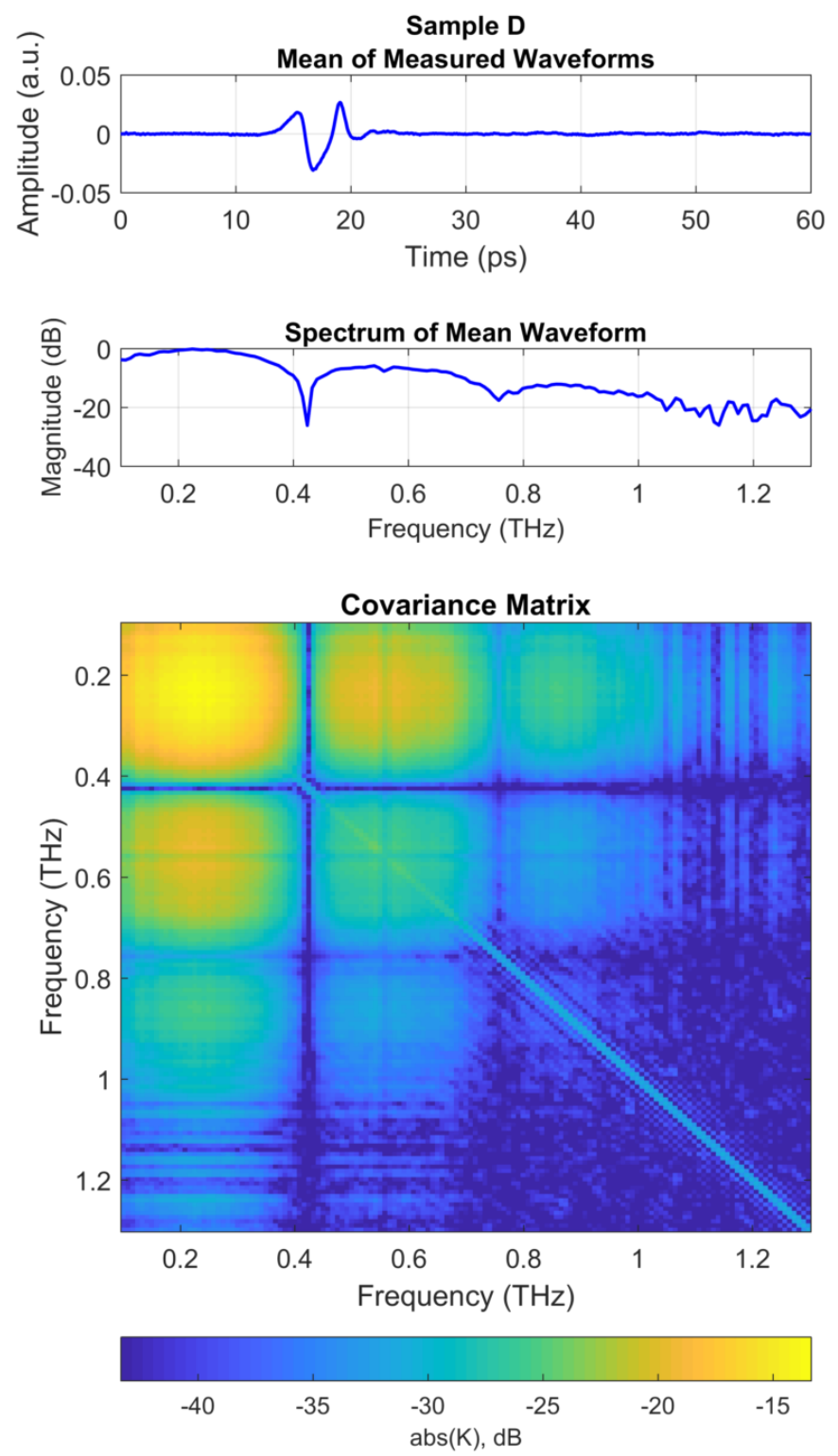

Figure B.1.4: Measured data for Sample D. Top Panel: Mean of measured THz waveforms. Note that reflected THz pulses 1, 2 and 3 (illustrated in Figure 4.2.1) all overlap on one another. Middle Panel: Spectrum of mean waveform in the top panel. Bottom Panel: Covariance matrix computed with Eq. 3.3.9 using the spectrum of each of the 300 measured waveforms. 


\section{B.2 Ambiguity Surfaces}

This section shows the ambiguity surfaces for each sample that was discussed in Section 4.2. Note, the ambiguity data for each image was normalized by its peak value. Therefore, the color axis for the ambiguity images always span the range between minimum and maximum possible values of $[0-1]$. Each of the ambiguity surfaces presented in this dissertation are normalized in the same way, which allows for direct comparisons of the ambiguity data from the Bartlett and MV processors for each sample. 

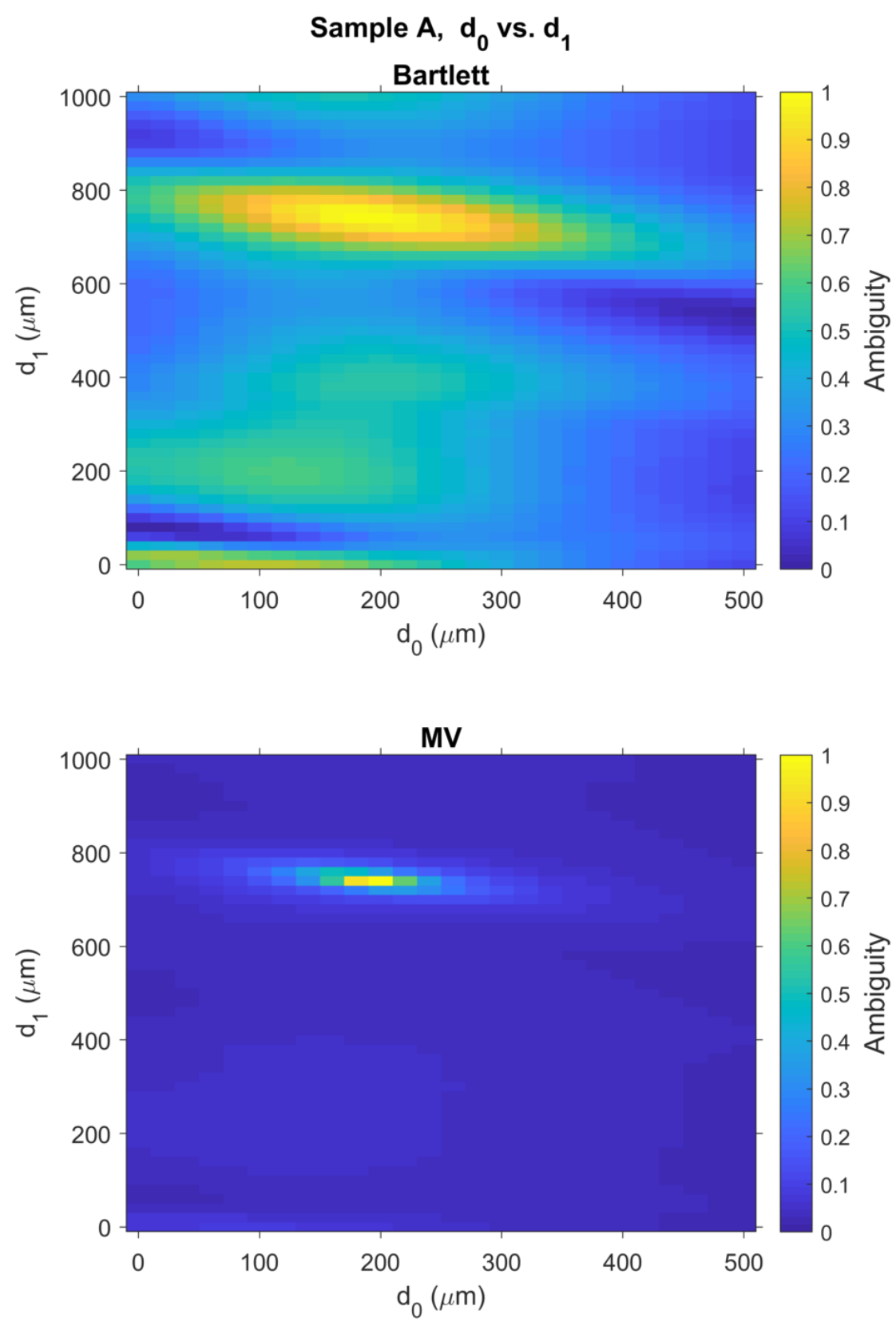

Figure B.2.1: Sample A: Matched field ambiguity surfaces provide estimates for the thickness of the calibration layer, $d_{0}$, and polycarbonate layer, $d_{1}$,

Top panel: Bartlett processor global maximum at $d_{0}=190 \mu \mathrm{m}$ and $d_{1}=750 \mu \mathrm{m}$.

Bottom panel: MV processor global maximum at $d_{0}=180 \mu \mathrm{m}$ and $d_{1}=750 \mu \mathrm{m}$. 

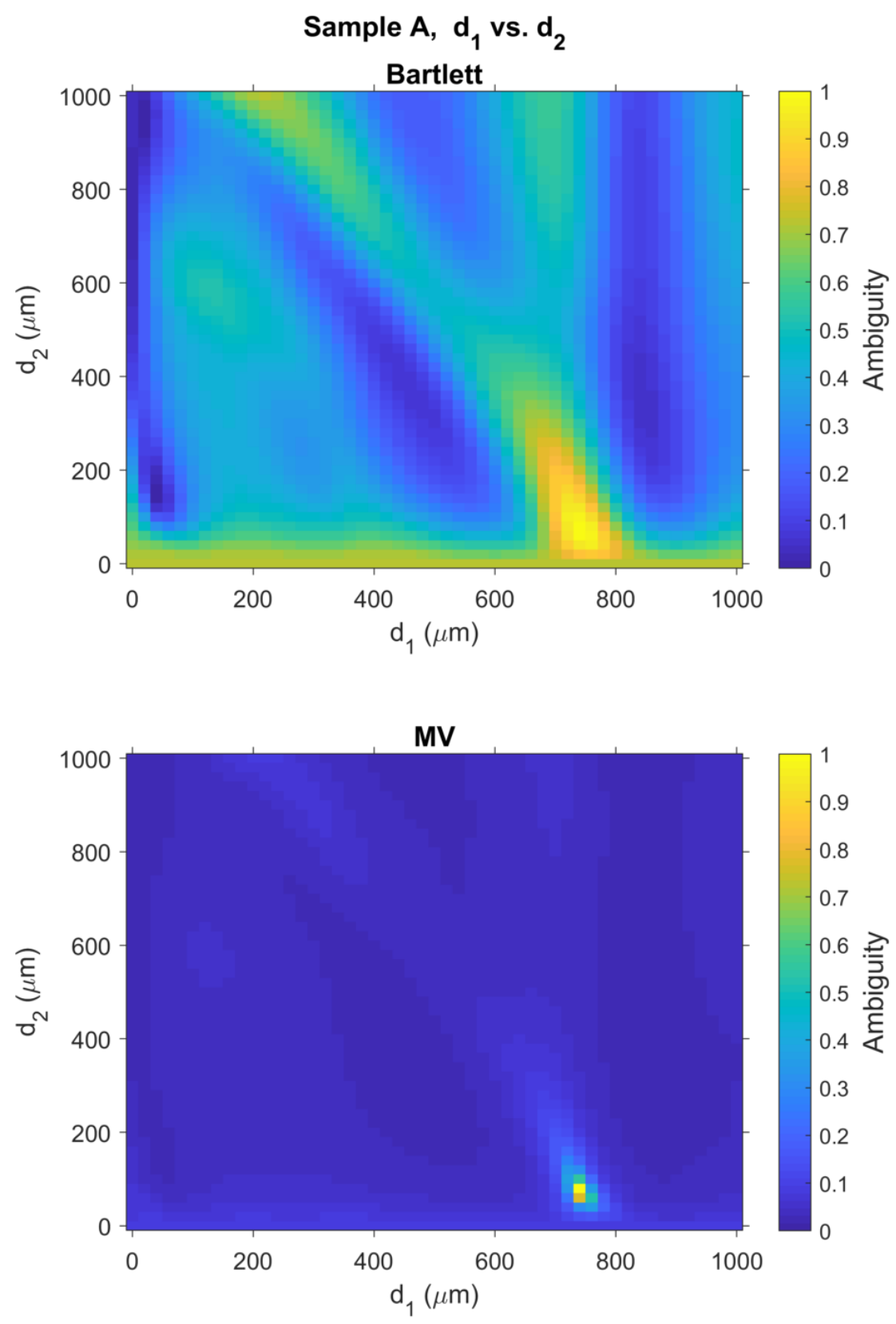

Figure B.2.2: Sample A: Matched field ambiguity surfaces provide estimates for the thickness of the polycarbonate layer, $d_{1}$ and the air gap, $d_{2}$.

Top panel: Bartlett processor global maximum at $d_{1}=750 \mu \mathrm{m}$ and $d_{2}=70 \mu \mathrm{m}$.

Bottom panel: MV processor global maximum at $d_{1}=750 \mu \mathrm{m}$ and $d_{2}=70 \mu \mathrm{m}$. 

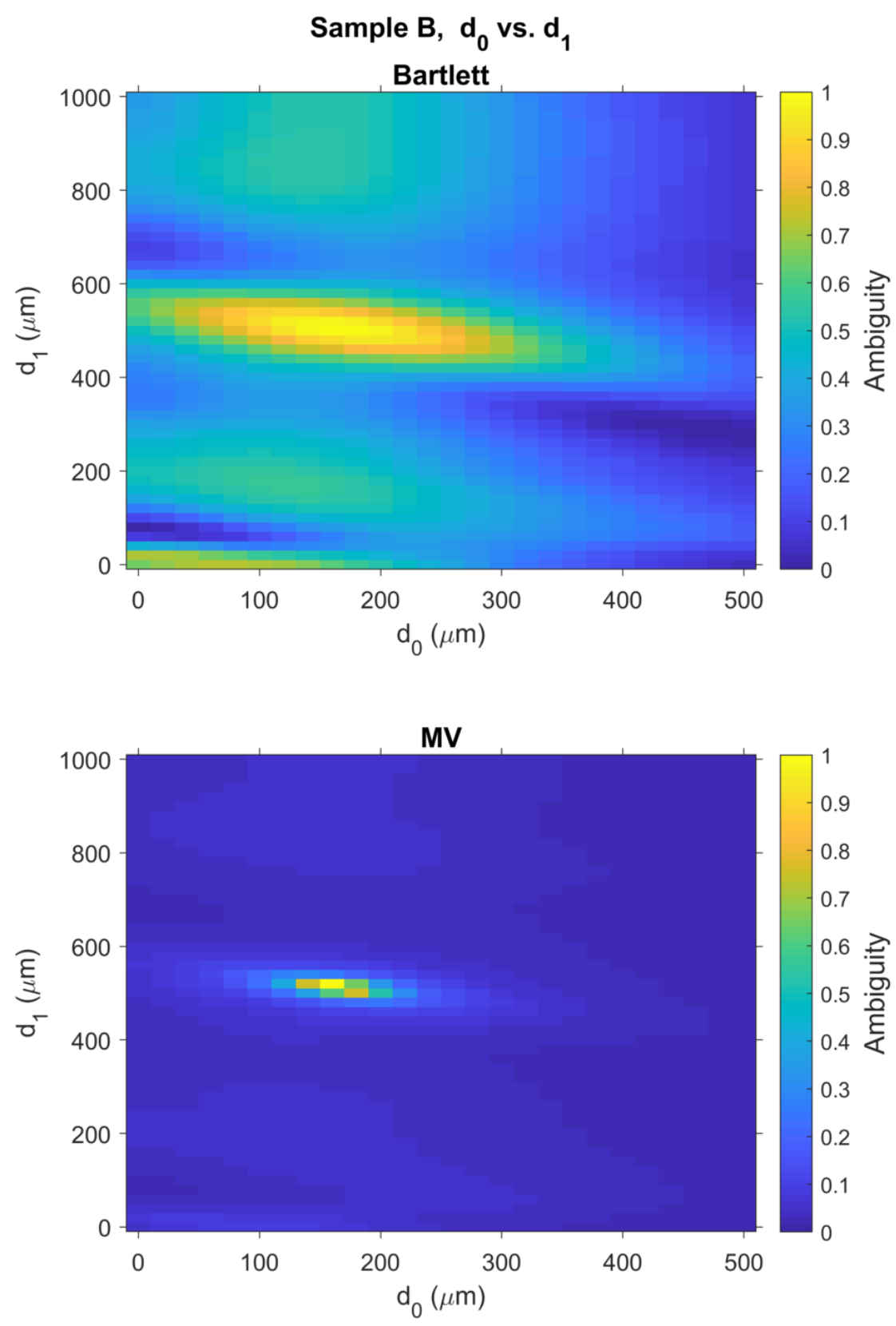

Figure B.2.3: Sample B: Matched field ambiguity surfaces provide estimates for the thickness of the calibration layer, $d_{0}$, and polycarbonate layer, $d_{1}$.

Top panel: Bartlett processor global maximum at $d_{0}=160 \mu \mathrm{m}$ and $d_{1}=510 \mu \mathrm{m}$. Bottom panel: MV processor global maximum at $d_{0}=160 \mu \mathrm{m}$ and $d_{1}=510 \mu \mathrm{m}$. 

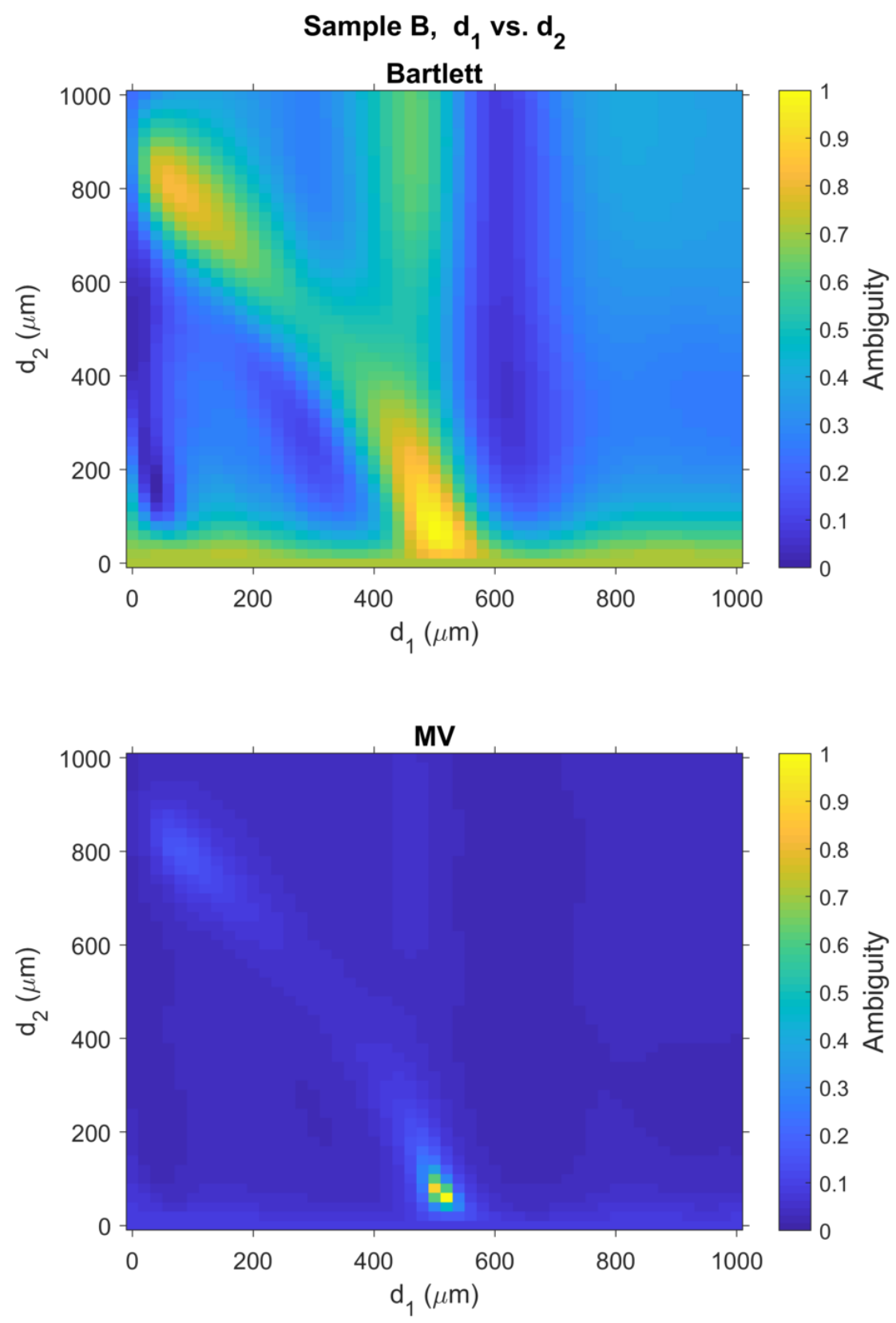

Figure B.2.4: Sample B: Matched field ambiguity surfaces provide estimates for the thickness of the polycarbonate layer, $d_{1}$ and the air gap, $d_{2}$, as illustrated in Figure 4.2.1 with layer thickness for Sample B given in Table 4.2.1.

Top panel: Bartlett processor global maximum at $d_{1}=510 \mu \mathrm{m}$ and $d_{2}=70 \mu \mathrm{m}$.

Bottom panel: MV processor global maximum at $d_{1}=510 \mu \mathrm{m}$ and $d_{2}=70 \mu \mathrm{m}$. 

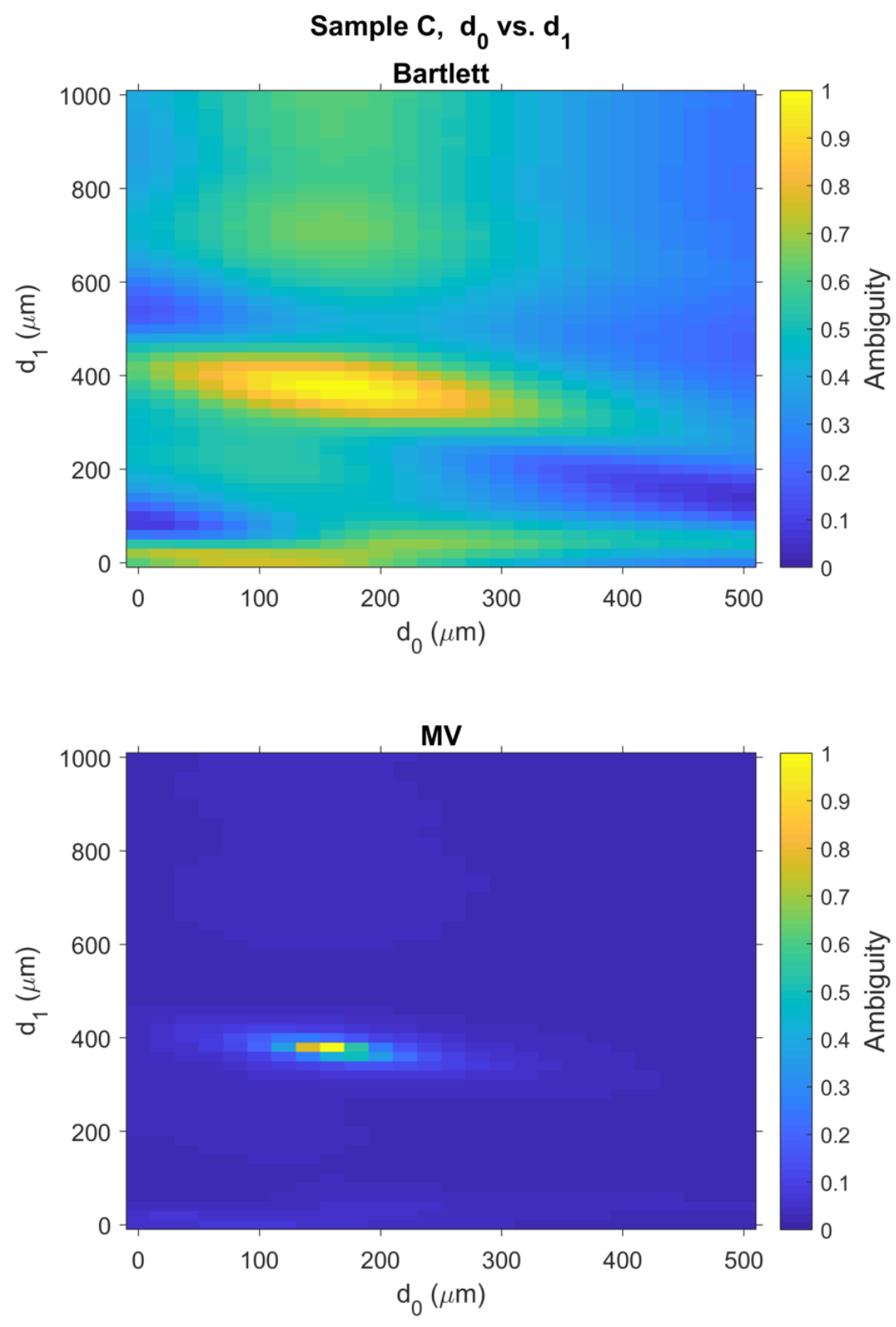

Figure B.2.5: Sample C: Matched field ambiguity surfaces provide estimates for the thickness of the calibration layer, $d_{0}$, and polycarbonate layer, $d_{1}$.

Top panel: Bartlett processor global maximum at $d_{0}=160 \mu \mathrm{m}$ and $d_{1}=380 \mu \mathrm{m}$. Bottom panel: MV processor global maximum at $d_{0}=160 \mu \mathrm{m}$ and $d_{1}=370 \mu \mathrm{m}$. 

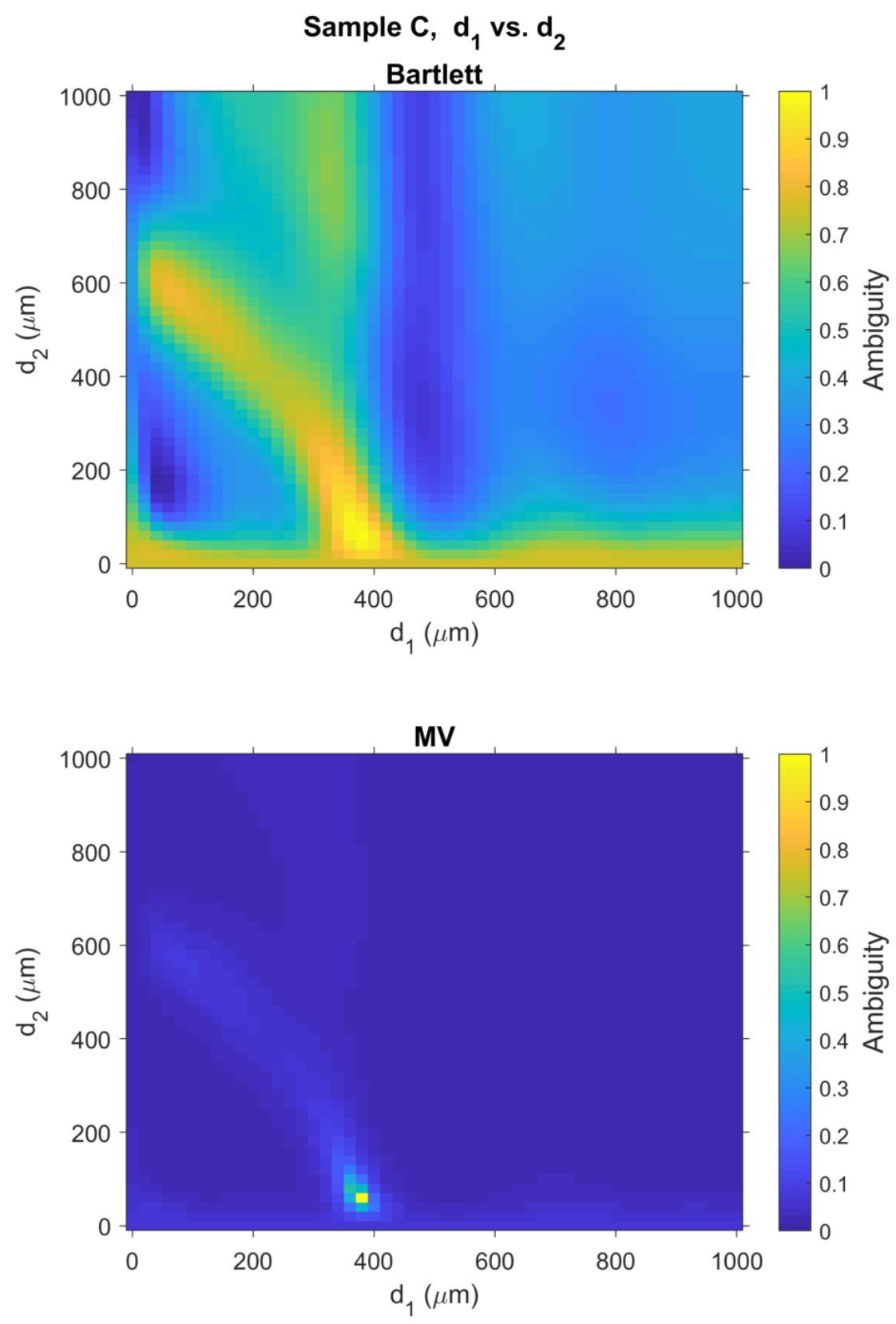

Figure B.2.6: Sample C: Matched field ambiguity surfaces provide estimates for the thickness of the polycarbonate layer, $d_{1}$ and the air gap, $d_{2}$.

Top panel: Bartlett processor global maximum at $d_{1}=380 \mu \mathrm{m}$ and $d_{2}=60 \mu \mathrm{m}$.

Bottom panel: MV processor global maximum at $d_{1}=370 \mu \mathrm{m}$ and $d_{2}=70 \mu \mathrm{m}$. 

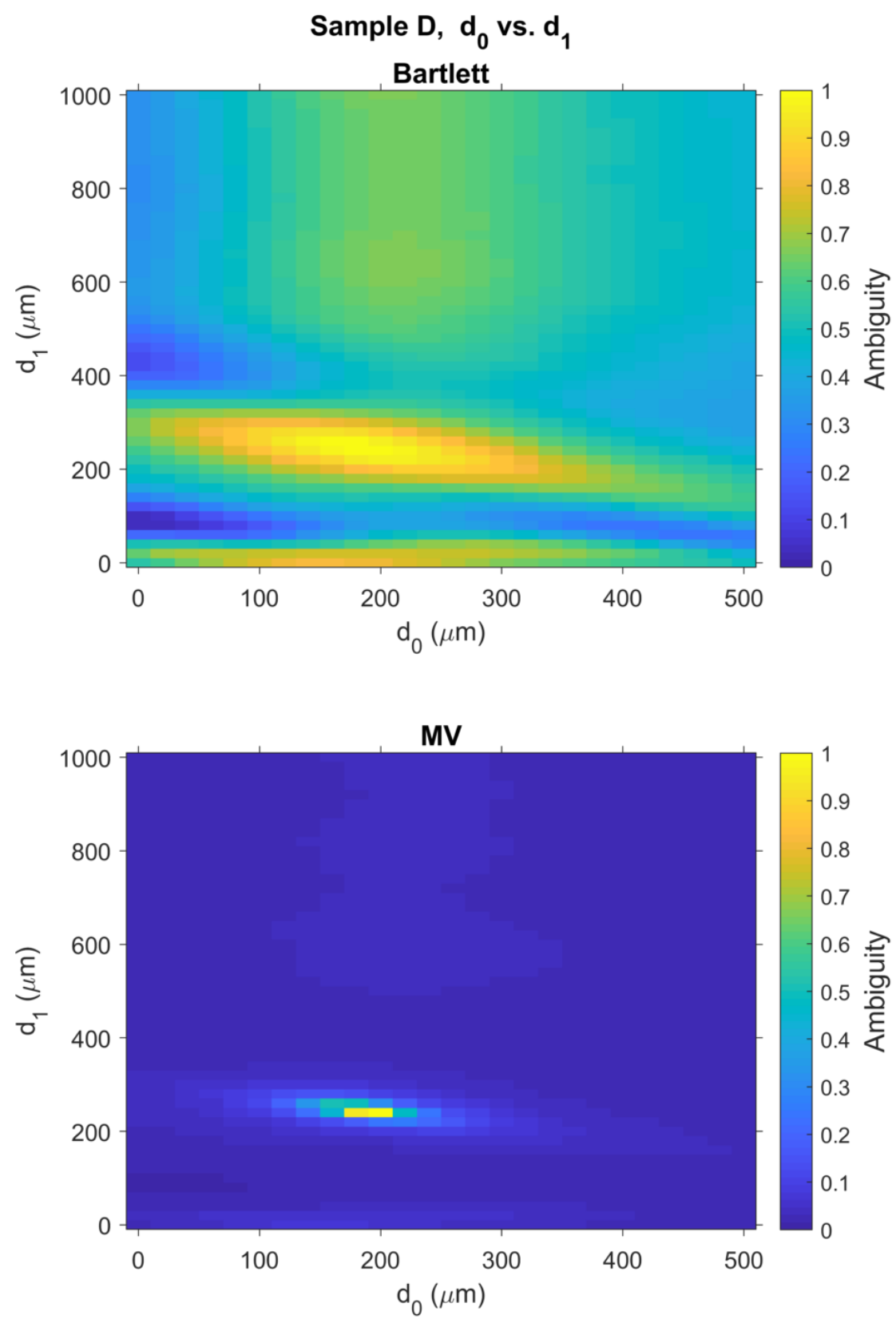

Figure B.2.7: Sample D: Matched field ambiguity surfaces provide estimates for the thickness of the calibration layer, $d_{0}$, and polycarbonate layer, $d_{1}$.

Top panel: Bartlett processor global maximum at $d_{0}=180 \mu \mathrm{m}$ and $d_{1}=250 \mu \mathrm{m}$. Bottom panel: MV processor global maximum $d_{0}=180 \mu \mathrm{m}$ and $d_{1}=240 \mu \mathrm{m}$. 

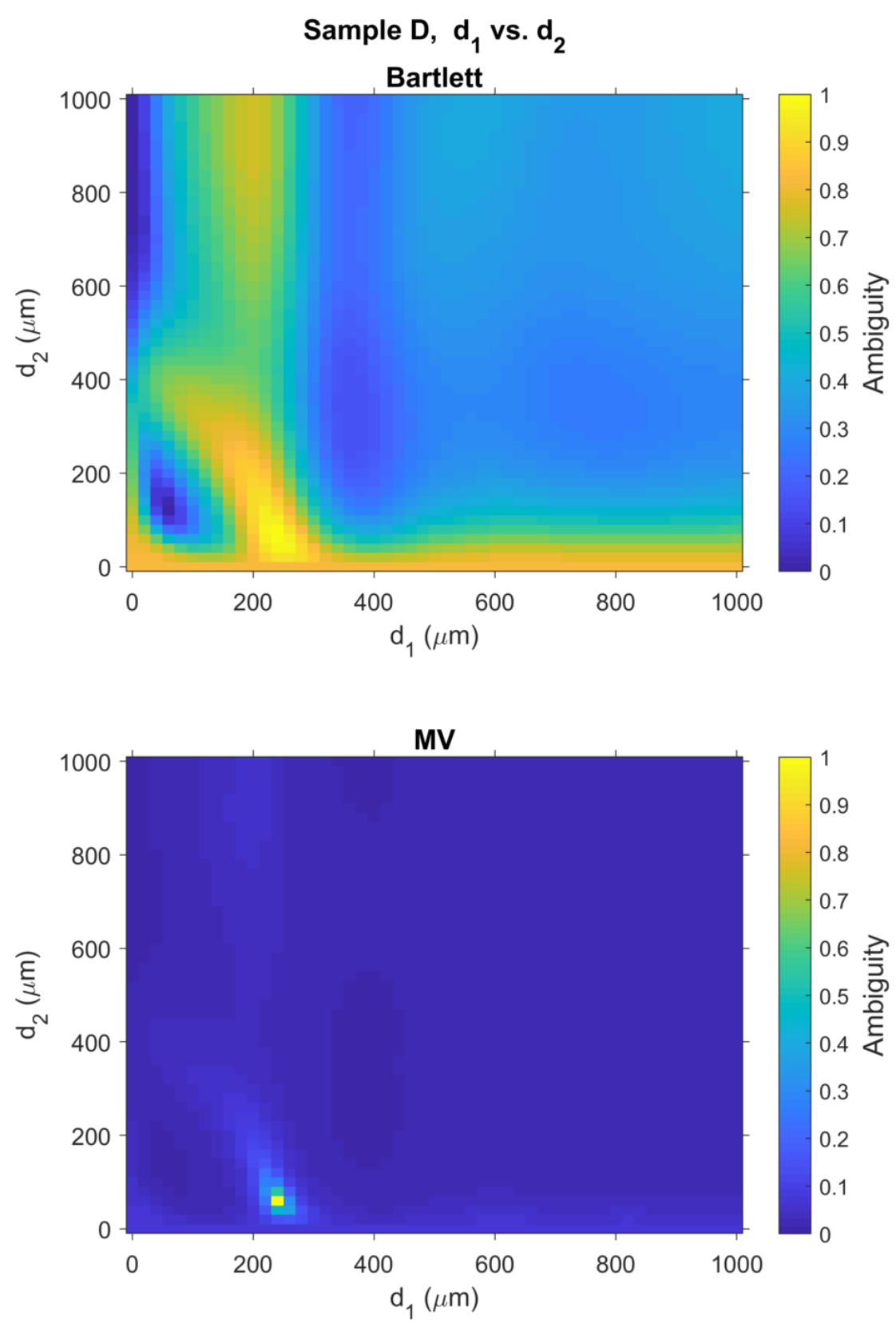

Figure B.2.8: Sample D: Matched field ambiguity surfaces provide estimates for the thickness of the polycarbonate layer, $d_{1}$ and the air gap, $d_{2}$.

Top panel: Bartlett processor global maximum at $d_{1}=250 \mu \mathrm{m}$ and $d_{2}=60 \mu \mathrm{m}$.

Bottom panel: MV processor global maximum at $d_{1}=240 \mu \mathrm{m}$ and $d_{2}=60 \mu \mathrm{m}$. 